\title{
$T$-systems with boundaries from network solutions
}

\author{
Philippe Di Francesco \\ Institut de Physique Théorique du CEA \\ Unité de Recherche associée du CNRS, \\ 91191 Gif sur Yvette Cedex, FRANCE. \\ philippe.di-francesco@cea.fr
}

\author{
Rinat Kedem \\ Department of Mathematics, \\ University of Illinois MC-382, \\ Urbana, IL 61821, U.S.A. \\ rinat@illinois.edu
}

Submitted: Aug 22, 2012; Accepted: Dec 23, 2012; Published: Jan 7, 2013

Mathematics Subject Classifications: 05C22;05E10;82B20

\begin{abstract}
In this paper, we use the network solution of the $A_{r} T$-system to derive that of the unrestricted $A_{\infty} T$-system, equivalent to the octahedron relation. We then present a method for implementing various boundary conditions on this system, which consists of picking initial data with suitable symmetries. The corresponding restricted $T$-systems are solved exactly in terms of networks. This gives a simple explanation for phenomena such as the Zamolodchikov periodicity property for $T$ systems (corresponding to the case $A_{\ell} \times A_{r}$ ) and a combinatorial interpretation for the positive Laurent property for the variables of the associated cluster algebra. We also explain the relation between the $T$-system wrapped on a torus and the higher pentagram maps of Gekhtman et al.
\end{abstract}

\section{Introduction}

\subsection{Discrete Integrable systems, positivity and periodicity}

Discrete integrable systems are evolution equations in a discrete time variable $k \in \mathbb{Z}$ that admit a sufficient number of conservation laws or integrals of motion, in the Liouville sense. In this note we concentrate essentially on the so-called $T$-system, which first arose in the context of integrable quantum spin chains, as a system of equations satisfied by the eigenvalues of transfer matrices of generalized Heisenberg magnets, with the symmetry of a given Lie algebra [18]. In the case of type $\mathrm{A}$, the $T$-system equation is also often referred to as the octahedron recurrence, and appears to be central in a number of combinatorial objects, such as: the $\lambda$-deformed determinant introduced by Robbins and Rumsey and its interplay with Alternating Sign Matrices [22]; the puzzles leading to the proof of positivity of Littlewood-Richardson coefficients for $s l_{n}[16]$; the partition function of domino tilings of 
the Aztec diamond $[8,23]$. Finally the $T$-system plays a central role as discrete integrable system, where it is referred to as the discrete Hirota equation [17]. Note that an interesting deformation of the T-system was considered by Nakajima [21]; the corresponding system is obeyed by the so-called q,t-characters of quantum affine algebras.

A new interpretation for the $T$-system arose from realizing that the corresponding discrete evolution could be viewed as a particular mutation in a suitably defined cluster algebra [5]. As such, it must satisfy the Laurent property, namely that any solution is a Laurent polynomial of any set of admissible initial data [9]. Moreover, the general positivity conjecture for cluster algebras would also imply that these Laurent polynomials have non-negative integer coefficients. Positivity for the unrestricted $T$-system expressed in terms of "flat" initial data follows from the interpretation of the solution as a positively weighted partition function for domino tilings of the Aztec diamond [23]. In the present paper, we first generalize this result to an explicit network solution for arbitrary initial data which we then adapt to include various types of boundary conditions. The network solutions display in particular the positive Laurent phenomenon.

Another fundamental property of $T$-systems was conjectured by Zamolodchikov [26] in the form of periodicity properties of the so-called $Y$-systems in the presence of special boundary conditions, which is a result of similar periodicity properties for the $T$-system. In a more general setting, the $Y$-system is attached to the Dynkin diagram $G$ of a Lie algebra, and the special boundaries are coded by another Dynkin diagram $G^{\prime}$, while the period of the system is given by $2\left(h_{G}+h_{G^{\prime}}\right)$, where $h_{G}$ is the Coxeter number of the corresponding algebra. This periodicity has been proved by many authors $[25,24,12,13,14]$ for the case when either or both $G, G^{\prime}$ are of $A$-type, culminating in the general proof of Keller [15] using category theory, for the case of any pair of Dynkin diagrams $G, G^{\prime}$. Note that the various methods of proof used in these works do not imply the positive Laurent phenomenon. The method presented in this paper for $\left(A_{r}, A_{\ell}\right)$, based on the explicit network solution, provides a simple combinatorial explanation for this property.

By analogy with the solutions of the so-called $Q$-systems [6], based on an explicit construction of conserved quantities, a first solution of $A_{r} T$-systems for particular periodic initial data surface was produced in terms of partition functions of paths with time- and space-dependent weights on some target graphs [7]. Finally the $A_{r} T$-system was explicitly solved [4] for arbitrary admissible initial conditions in terms of weighted path models on specific networks, coded by the geometry of the initial data surface.

The aim of this paper is to use the network solution of the $A_{r}$ case to derive properties of solutions of $T$-systems with different kinds of boundary conditions. We start with the unrestricted $T$-system: by using the formulas for $A_{r}$ for $r$ large enough, we show that any fixed unrestricted $T$-system solution can be expressed in a compact form, as a principal minor of a positive network matrix coded by the geometry of the initial conditions.

To address other boundary conditions, our strategy consists in identifying suitable initial data for the $A_{r}$ system, that imply the presence of the desired boundaries, such as walls along which the values of the $T$-system solution must be equal to 1 . Once these are identified, we must plug them into the network solution of the $A_{r}$ system. The network solution happens to behave nicely under these symmetries, and can be reduced to explicit 
positive expressions in all cases. As a result, we obtain closed formulas for the solutions of the $T$-system with various boundary conditions.

\subsection{T-system: definitions}

Let us now give a few definitions regarding the $T$-system and its various boundary conditions.

\subsubsection{The unrestricted $A_{\infty} T$-system}

The unrestricted $A_{\infty} T$-system, also called octahedron recurrence, is the following system for formal variables $T_{i, j, k}, i, j, k \in \mathbb{Z}$ :

$$
T_{i, j, k+1} T_{i, j, k-1}=T_{i, j+1, k} T_{i, j-1, k}+T_{i+1, j, k} T_{i-1, j, k} \quad(i, j, k \in \mathbb{Z}) .
$$

The system splits into two independent systems corresponding to a fixed parity of $i+j+k$. From now on we restrict ourselves to $T_{i, j, k}$ with $i+j+k=0 \bmod 2$.

This system can be considered as a three-term recursion relation in $k$. As such it has the following sets of admissible initial conditions, attached to a stepped surface defined as follows.

Definition 1.1. A stepped surface in the variables $(i, j, k) \in \mathbb{Z}^{3}$ is a set:

$$
\mathbf{k}=\left\{\left(i, j, k_{i, j}\right) \in \mathbb{Z}^{3}: i+j+k_{i, j}=0 \bmod 2 \text { and }\left|k_{i, j}-k_{i^{\prime}, j^{\prime}}\right|=1 \text { if }\left|i-i^{\prime}\right|+\left|j-j^{\prime}\right|=1\right\} \text {. }
$$

To any such stepped surface, we attach the initial condition:

$$
X_{\mathbf{k}}(\mathbf{t}):\left\{T_{i, j, k_{i, j}}=t_{i, j} \quad(i, j \in \mathbb{Z})\right\}
$$

for some formal variables $\mathbf{t}=\left\{t_{i, j}\right\}_{i, j \in \mathbb{Z}}$, which we refer to as initial data/values along the surface $\mathbf{k}$.

The interplay between these admissible initial conditions is best understood if we interpret the relation (1.1) as a mutation relation for the cluster algebra related to the $T$ system [5]. In this setting, the admissible initial data are cluster variables $x_{\mathbf{k}}=\left(t_{i, j}\right)_{i, j \in \mathbb{Z}}$ in a seed of the cluster algebra, and a mutation $\mu_{i, j}$ is simply one application of the relation (1.1) where $k=k_{i, j+1}=k_{i, j-1}=k_{i+1, j}=k_{i-1, j}$ and either $k_{i, j}=k-1$ (forward mutation) or $k_{i, j}=k+1$ (backward mutation). The mutation $\mu_{i, j}$ sends the surface $\mathbf{k}$ to a new surface $\mathbf{k}^{\prime}$ such that $k_{a, b}^{\prime}=k_{a, b}+2 \epsilon \delta_{a, i} \delta_{b, j}$ with $\epsilon=1$ for a forward mutation, and $\epsilon=-1$ for a backward mutation. Accordingly, the initial data along the surface $\mathbf{k}$ is transformed into initial data along $\mathbf{k}^{\prime}$ by keeping the same values $t_{a, b}^{\prime}=t_{a, b}$ except for $a=i$ and $b=j$, where

$$
t_{i, j}^{\prime}=\frac{t_{i, j-1} t_{i, j+1}+t_{i-1, j} t_{i+1, j}}{t_{i, j}} .
$$


The following is a pictorial representation of a forward mutation:

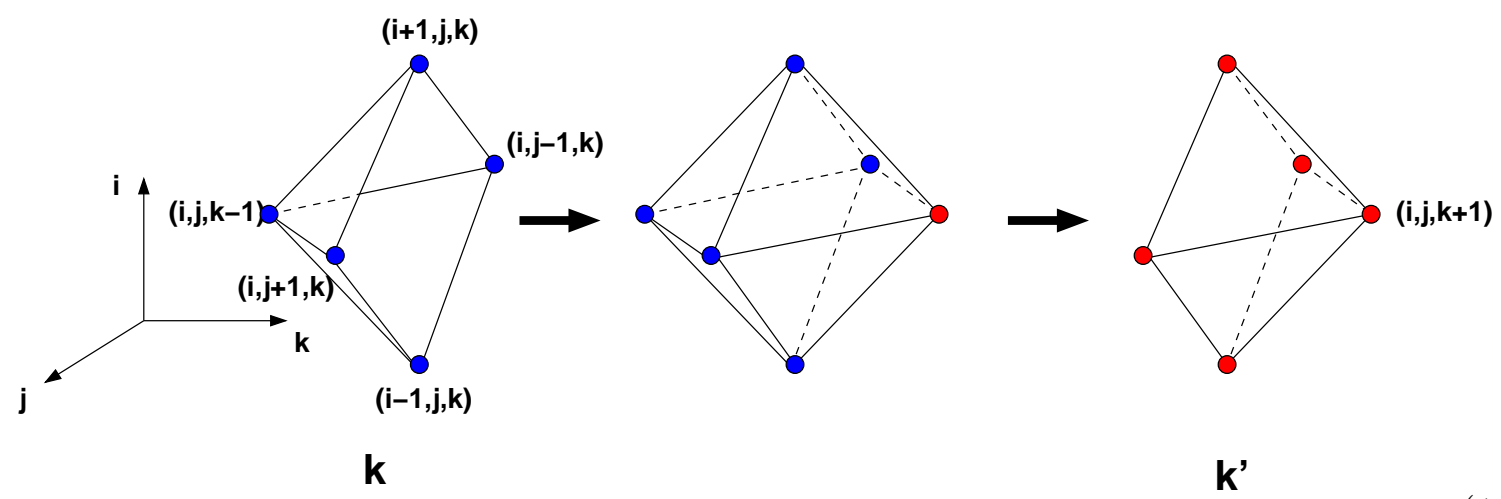

It shows how the mutated surface $\mathbf{k}^{\prime}$ differs from $\mathbf{k}$ by one point, which is the sixth point $(i, j, k+1)$ of the incomplete octahedron $(i, j, k-1),(i, j+1, k),(i, j-1, k),(i+1, j, k),(i-$ $1, j, k)$, hence the name "octahedron" equation often used for (1.1). Iterating mutations on a given stepped surface $\mathbf{k}$, we may attain any other stepped surface $\mathbf{k}^{\prime}$.

In the following, unless otherwise stated, we will refer to the fundamental stepped surface as "flat" stepped surface $\mathbf{k}_{0}$ with $k_{i, j}^{(0)}=i+j \bmod 2$.

\subsubsection{The $T$-system for $A_{r}$}

In the case of the $A_{r}$ Lie algebra, the $T$-system (1.1) is restricted to values of $i \in[1, r]$ and is subject to the boundary condition

$$
T_{0, j, k}=T_{r+1, j, k}=1 \quad \text { for all } j, k \in \mathbb{Z} .
$$

The system (1.1-1.6) can still be considered as a three-term recursion relation in $k$. The corresponding admissible initial data are attached to infinite strip-like stepped surfaces $\mathbf{k}=\left(i, j, k_{i, j}\right)_{i \in[1, r] ; j \in \mathbb{Z}}$ such that $k_{i, j} \in \mathbb{Z}, i+j+k_{i, j}=0 \bmod 2$, and $\left|k_{i+1, j}-k_{i, j}\right|=$ $\left|k_{i, j+1}-k_{i, j}\right|=1$ for all $i, j$. To each such $\mathbf{k}$, we associate the initial conditions:

$$
X_{\mathbf{k}}(\mathbf{t}):\left\{T_{i, j, k_{i, j}}=t_{i, j} \quad(i \in[1, r] ; j \in \mathbb{Z})\right\}
$$

for some formal variables $\mathbf{t}=\left\{t_{i, j}\right\}_{i \in[1, r] ; j \in \mathbb{Z} \text {. }}$

The fundamental "flat" stepped surface, still denoted by $\mathbf{k}_{0}$ now has $k_{i, j}^{(0)}=i+j \bmod$ 2 , for $i \in[1, r], j \in \mathbb{Z}$.

The following useful lemma allows to eliminate the $T_{i, j, k}$ for $i>1$ in terms of the $T_{1, j^{\prime}, k^{\prime}}$ s.

Lemma 1.2. [2] The solutions $T_{i, j, k}$ to the $A_{r} T$-system may be expressed for $i=1, \ldots, r$ as the following "discrete Wronskian" determinants involving only $T_{1, j^{\prime}, k^{\prime}}$ 's:

$$
T_{i, j, k}=\operatorname{det}_{1 \leqslant a, b \leqslant i}\left(T_{1, j+a-b, k+a+b-i-1}\right) .
$$




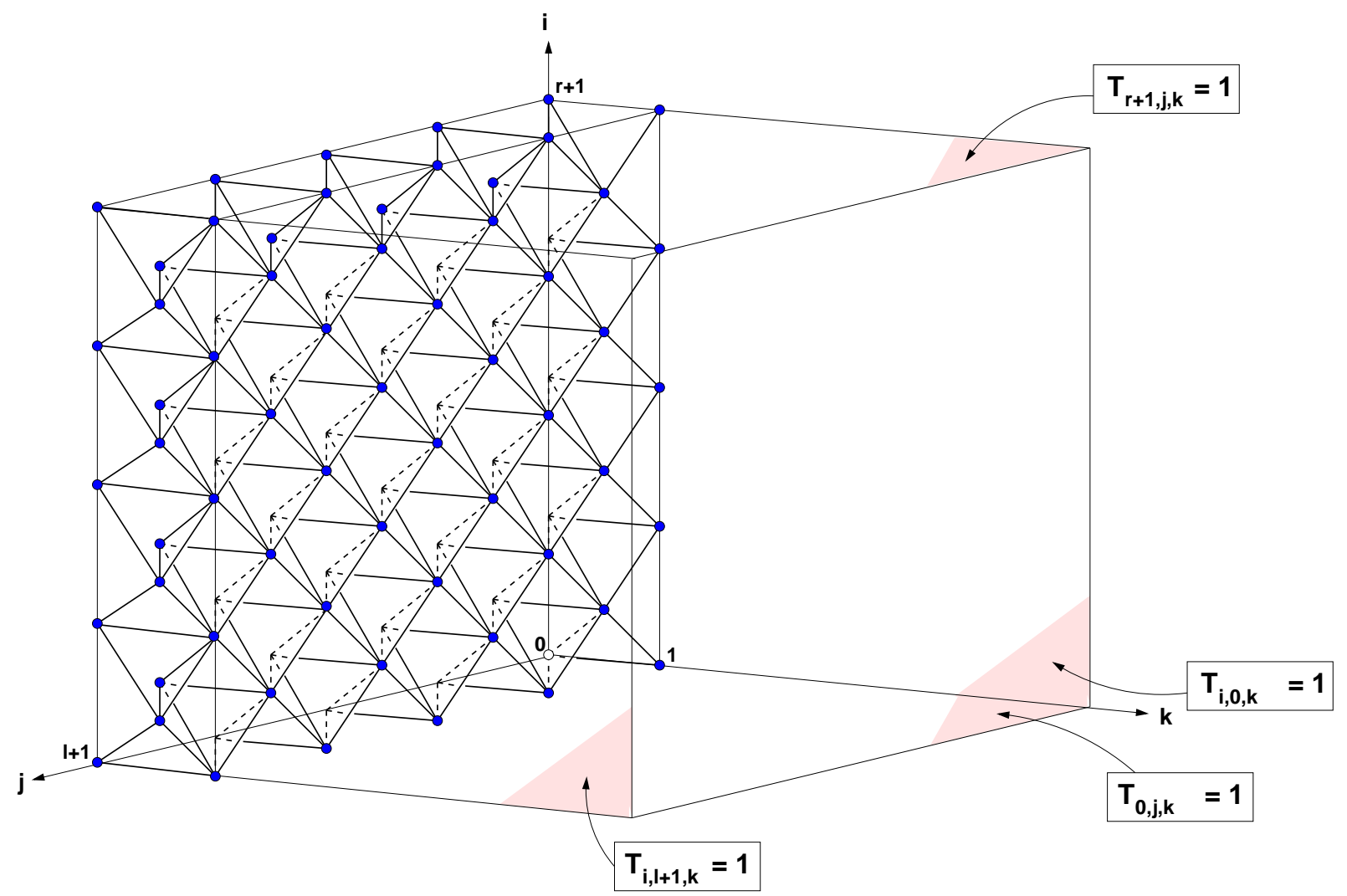

Figure 1: The $\ell$-restricted $T$-system geometry. We have represented in $(i, j, k)$-space the four walls along which we set $T_{i, j, k}=1$, as well as the flat initial data stepped surface $\mathbf{k}_{0}$ with vertices in the planes $k=0$ and $k=1$. 
Proof. This is a direct consequence of the Desnanot-Jacobi identity relating the determinant $|M|$ of any $N \times N$ matrix $M$ to its minors $|M|_{i_{1}}^{j_{1}}$ and $|M|_{i_{1}, i_{2}}^{j_{1}, j_{2}}$ obtained respectively by erasing row $i_{1}$ and column $j_{1}$ of $M$ or rows $i_{1}, i_{2}$ and columns $j_{1}, j_{2}$ of $M$ :

$$
|M| \times|M|_{1, N}^{1, N}=|M|_{1}^{1} \times|M|_{N}^{N}-|M|_{N}^{1} \times|M|_{1}^{N},
$$

with the convention that the determinant of a $0 \times 0$ matrix is 1 . The lemma follows by taking the $(i+1) \times(i+1)$ matrix $M$ with entries $M_{a, b}=T_{1, j+a-b, k+a+b-i-2}, a, b=$ $1,2, \ldots, i+1$.

We will also consider the $A_{r} T$-system with so-called $\ell$-restricted boundary conditions, in which we restrict the range of $j \in[0, \ell+1]$ and we impose

$$
T_{i, 0, k}=T_{i, \ell+1, k}=1 \quad(i \in[1, r] ; k \in \mathbb{Z}) .
$$

In this case the initial data is also restricted to a finite sequence $\mathbf{t}=\left\{t_{i, j}\right\}_{i \in[1, r] ; j \in[1, \ell]}$, and the associated initial conditions read:

$$
X_{\mathbf{k}}(\mathbf{t}):\left\{T_{i, j, k_{i, j}}=t_{i, j} \quad(i \in[1, r] ; j \in[1, \ell])\right\} .
$$

The boundary conditions and flat surface initial data for the $\ell$-restricted $T$-system are sketched in Fig.1.

\subsection{Main results}

In this paper we explore the effect of imposing various boundary conditions of the $T$ system.

For the unrestricted $T$-system, we derive a compact explicit expression for the solution, first in terms of the initial data $X_{\mathbf{k}_{0}}$ (1.3) along the flat stepped surface $\mathbf{k}_{0}$ (Theorem 3.9 and Corollary 3.11). This is then generalized to arbitrary initial data $X_{k}$ (Theorem 3.12). As a consequence, we have:

Theorem 1.3. The solution $T_{i, j, k}$ of the unrestricted $T$-system (1.1) with arbitrary initial conditions $X_{\mathbf{k}}(1.3)$ is a Laurent polynomial of the initial values $\left\{t_{i, j}\right\}$ with non-negative integer coefficients.

This extends the result of [23], corresponding to $\mathbf{k}=\mathbf{k}_{0}$ in our language.

Next we consider the $A_{r} T$-system in different geometries, first in a right or left halfplane bordered by a "wall" $j=$ constant, along which the value of $T_{i, j, k}$ is fixed to 1 . We show that the solutions of such systems coincide with that of the one without a wall, provided we pick initial data obeying certain symmetry relations (Theorems 5.6 and 5.7). We also show that the solutions of the two-wall $\ell$-restricted $A_{r} T$-system coincide with that of the system without walls but with initial data obeying multiple reflection symmetries inherited from the two half-plane cases (Theorem 5.8). In all cases, we have an explicit formula for the solution in terms of the initial data. This will allow us in particular to establish the following two results on the solutions of the $\ell$-restricted $A_{r} T$-system. 
Theorem 1.4. The solution of the $\ell$-restricted $A_{r} T$ system with arbitrary initial conditions $X_{\mathbf{k}}$ satisfies the following periodicity condition:

$$
T_{i, j, k+N}=T_{i, j, k} \quad(i \in[1, r] ; j, k \in \mathbb{Z})
$$

with period $N=2(\ell+r+2)$.

Theorem 1.5. The solution $T_{i, j, k}$ of the $\ell$-restricted $A_{r} T$ system with initial conditions $X_{\mathbf{k}_{0}}$ (1.11) along the "flat" stepped surface $\mathbf{k}_{0}$ is a Laurent polynomial of the initial values $\left\{t_{i, j}\right\}$ with non-negative integer coefficients.

In all cases, the positivity of the coefficients will arise from a combinatorial interpretation, as counting families of non-intersecting paths on suitable network graphs.

\subsection{Outline}

The paper is organized as follows.

In Sect.2, we recall the solution of the $A_{r} T$-system for an arbitrary initial data stepped surface $\mathbf{k}$. The solution is expressed in terms of paths on networks. The latter are made of elementary "chips" associated to $2 \times 2$ matrices $U, V$ whose arrangement is coded by the initial data stepped surface $\mathbf{k}$, and whose entries are Laurent monomials of the initial data values along $\mathbf{k}$.

This solution is exploited in Sect.3 to derive the solution of the unrestricted $T$-system for an arbitrary initial data stepped surface $\mathbf{k}$ (Theorem 3.12). We find that $T_{i, j, k}$ is equal, up to simple factors of the initial data, to a principal minor of a network matrix corresponding to the shadow of the point $(i, j, k)$ onto the stepped surface $\mathbf{k}$, namely the intersection of $\mathbf{k}$ and the pyramid $\{(x, y, z)$ such that $|i-x|+|j-y| \leqslant|k-z|\}$. Theorem 1.3 follows from this expression.

Sects. 4 and 5 are devoted to the study of the $\ell$-restricted $A_{r} T$ system solutions. For pedagogical reasons, we first treat the case $r=1$ completely in Sect.4, where we derive network formulas for the general solution of the $A_{1} T$-system. We first treat the case of the right (resp. left) half-plane $A_{1} T$-system, which correspond to imposing a wall-type boundary condition on $T_{1, j, k}$ along the "wall" $j=0$ (resp. $j=\ell+1$ ) and restricting the range of $j$ to the half-plane $j>0$ (resp. $j<\ell+1$ ). The general strategy is to consider a full plane $A_{1} T$-system, and to engineer both its initial data stepped surface and initial values to ensure that the solution coincides with that of the half-plane in the relevant range of $j$. This allows to use the general full plane network solution to derive results in the half-plane geometry, in particular to establish the positive Laurent property of the solution, first for the "flat" initial data stepped surface $\mathbf{k}_{0}$ (Theorems 4.7 and 4.5), and then for general $\mathbf{k}$ (Theorem 4.9). Superimposing both half-plane conditions leads to the $\ell$-restricted $A_{1} T$-system, whose network solution leads to the $A_{1}$ version of the periodicity property of Theorem 1.4 (Theorem 4.2). This solution allows to prove the $A_{1}$ version of the Laurent positivity of Theorem 1.5 (Theorem 4.3 for the flat stepped surface $\mathbf{k}_{0}$ and Theorem 4.4 for the general stepped surface $\mathbf{k}$ ). 
The same strategy is then applied to the case of general $r$ in Sect. 5, namely we impose special restrictions to the initial data stepped surface and values of the full space $A_{r} T$ system so as to mimic wall-type boundaries (left or right half-space) geometries (Theorem 5.6 and Corollary 5.7 for the stepped surface $\mathbf{k}_{0}$ ). Finally, by superimposing the two, we obtain the $\ell$-restricted two-wall boundary geometry (Theorem 5.8 for $\mathbf{k}_{0}$ ). These special restrictions however impose the vanishing of the initial values $t_{i, j}$ within square domains of the form $(i, j) \in[1, r] \times([-r,-1] \bmod \ell+r+2)$, which create potential singularities in the corresponding network matrices. To repair this, we use a regularization procedure detailed in Sect.5.2, by assigning special non-zero values within these squares, to be sent to zero in the end. With this trick, all formulas are well-defined and the relevant limits yield the solutions in half-space (Sect.5.5) and $\ell$-restricted geometries (Sects.5.6 and 5.7).

In the concluding Section 6 we consider other types of boundary conditions on the $T$ system related to Frieze patterns of the plane [1], pentagram [10] and higher pentagram maps [11]. We show that the latter are connected to $T$-systems wrapped on a torus, namely with doubly-periodic initial data. Finally we discuss generalized cut-like boundary conditions and formulate some further positivity conjectures.

\section{Networks and the $A_{r} T$-system solution}

In this section we recall the network solution [4] of the infinite $A_{r} T$-system, not subject to the $\ell$-restriction. The basic building blocks are matrices $U$ and $V$, which form the elementary "chips" of a network.

\subsection{Definitions and properties of the matrices $U$ and $V$}

Define the $2 \times 2$ matrices

$$
U(a, b, c)=\left(\begin{array}{cc}
1 & 0 \\
\frac{c}{b} & \frac{a}{b}
\end{array}\right), \quad V(a, b, c)=\left(\begin{array}{cc}
\frac{b}{c} & \frac{a}{c} \\
0 & 1
\end{array}\right) .
$$

These are embedded in $G L_{r+1}$ in the standard way: Given $i \in[1, r]$, define $U_{i}(a, b, c)$ as the $(r+1) \times(r+1)$ matrix with entries

$$
\left(U_{i}(a, b, c)\right)_{k, \ell}= \begin{cases}(U(a, b, c))_{k-i+1, \ell-i+1}, & \text { if } k, \ell \in\{i, i+1\} \\ \delta_{k, \ell} & \text { otherwise }\end{cases}
$$

and similarly for $V_{i}(a, b, c)$.

These elementary matrices have the following important properties:

$$
\begin{aligned}
U_{i}(a, b, c) V_{i+1}(b, c, d) & =V_{i+1}(a, c, d) U_{i}(a, b, d) \\
V_{i}(a, b, c) U_{i+1}(d, e, f) & =U_{i+1}(d, e, f) V_{i}(a, b, c) \\
U(a, b, u) V(v, b, c) & =V\left(v, a, b^{\prime}\right) U\left(b^{\prime}, c, u\right) \quad \text { iff } \quad b b^{\prime}=u v+a c
\end{aligned}
$$

The third relation is crucial: It gives a representation of the mutation relation of the $T$-system (1.4-1.5) via a matrix exchange identity. 


\subsection{Pictorial representations}

In this paper we will use two pictorial representations of the elementary network matrices.

\subsubsection{Pictorial representation I}

The matrices $U_{i}(a, b, c)$ and $V_{i}(a, b, c)$ are represented as bicolored lozenges:

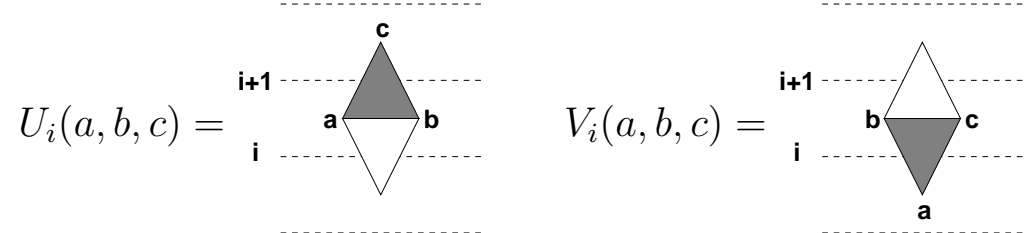

A product of matrices of $U$ and $V$ type is represented by drawing the corresponding lozenges in the same order from left to right, and identifying the edges of the triangles whenever no other object sits inbetween. This forms a triangulation of some region in the plane.

The relations satisfied by the elementary network matrices can be represented pictorially as follows. Property (2.3) is (we allow the triangles to be slightly deformed):

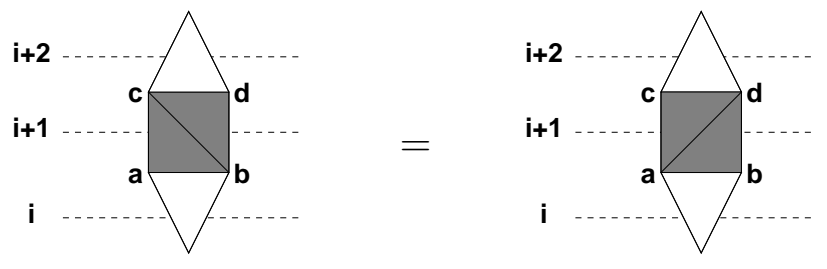

Property (2.4) is
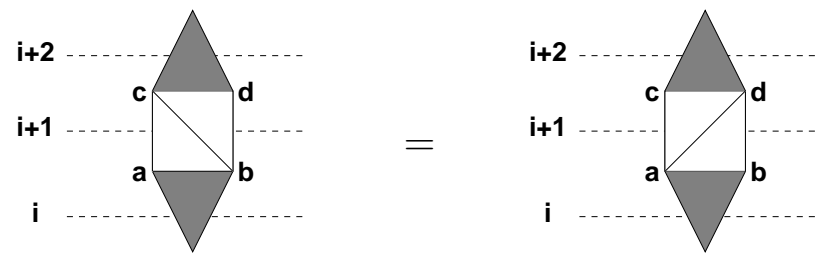

and the mutation $(2.5)$ is
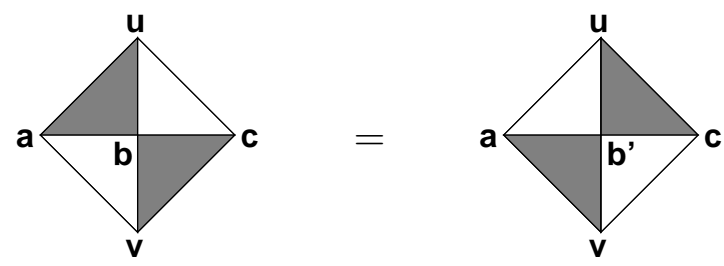


\subsubsection{Pictorial representation II}

The second useful pictorial representation is as network chips. The picture is as follows:

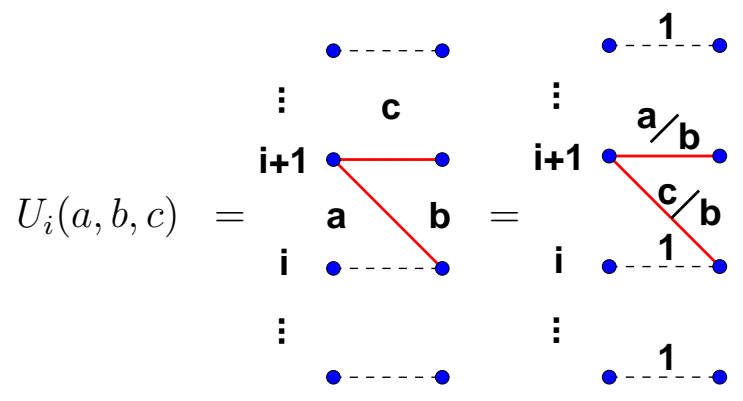

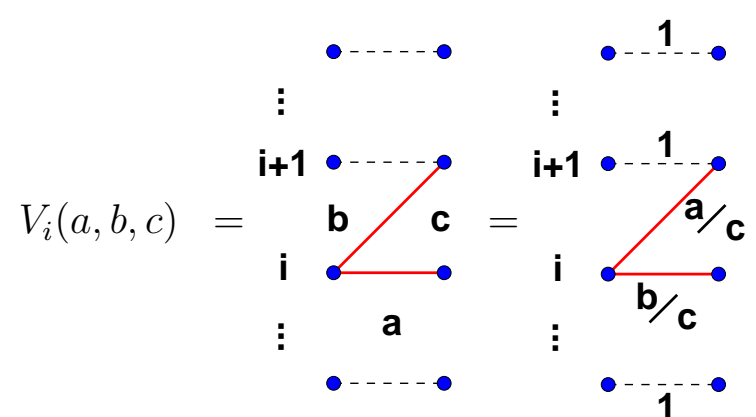

where $a, b, c$ are represented on the faces of a graph in the left picture. The edges, which are all oriented from left to right (for simplicity, this orientation is omitted in the pictures) are weighted by the matrix element $(j, k)$ for an edge from $j$ to $k$. By convention, dashed edges carry the weight 1 . We have indicated the weights of the edges in the right picture.

In terms of the network chips, property (2.3) can be illustrated as

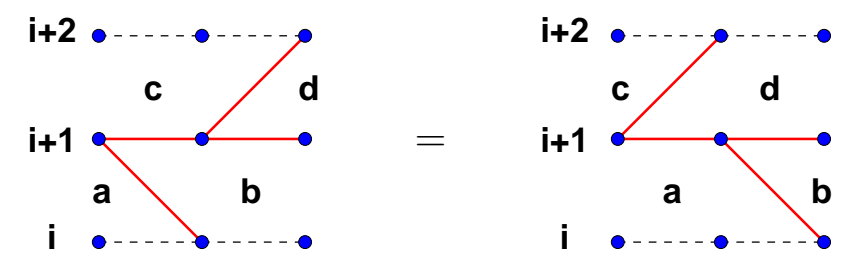

Property $(2.4)$ is
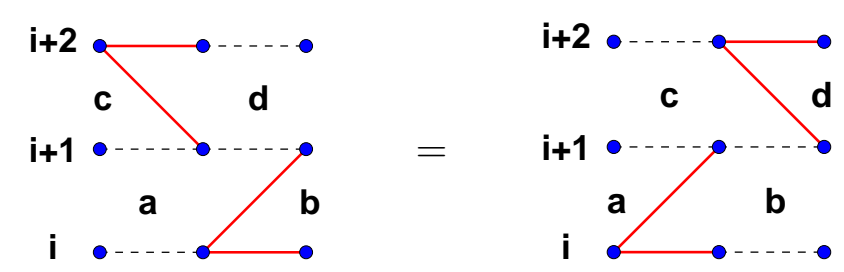

The mutation (2.5) is illustrated as

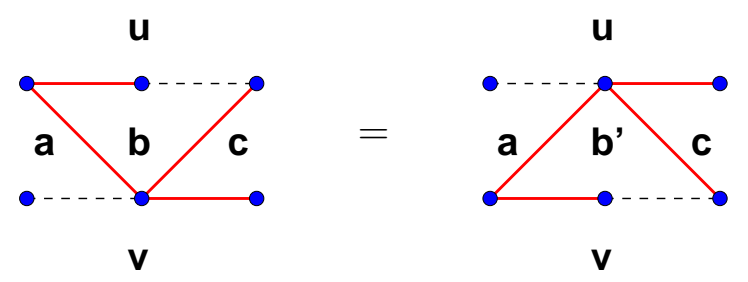




\subsection{Network Matrix}

\subsubsection{Definition}

Given some initial condition $X_{\mathbf{k}}$ as in (1.3), define the $(r+1) \times(r+1)$ matrices

$$
\begin{aligned}
N_{i, j} & =\left\{\begin{array}{ll}
U_{i}\left(t_{i, j-1}, t_{i, j}, t_{i+1, j-1}\right), & \text { if } k_{i, j-1}=k_{i, j}-1 ; \\
V_{i}\left(t_{i-1, j}, t_{i, j-1}, t_{i, j}\right) & \text { otherwise. }
\end{array} \quad(i \in[1, r] ; j \in \mathbb{Z})\right. \\
M_{0, j} & =\mathbb{I} \\
M_{i, j} & =\left\{\begin{array}{ll}
N_{i, j} M_{i-1, j}, & \text { if } k_{i, j}=k_{i-1, j-1}, k_{i-1, j} \neq k_{i, j-1} ; \\
M_{i-1, j} N_{i, j} & \text { otherwise. }
\end{array} \quad(i \in[1, r] ; j \in \mathbb{Z})\right. \\
N_{j} & =M_{r, j}
\end{aligned}
$$

The network matrix corresponding to the initial condition $X_{\mathbf{k}}$ is

$$
N\left(j_{0}, j_{1}\right)=\prod_{j=j_{0}+1}^{j_{1}} N_{j} \quad\left(j_{0} \leqslant j_{1}\right)
$$

with the convention that $t_{0, j}=t_{r+1, j}=1$ and $N(j, j)=\mathbb{I}$. The order of multiplication in (2.14) is according to increasing values of $j$. We may think of $N\left(j_{0}, j_{1}\right)$ as a network matrix corresponding to a slice of the initial data surface, containing the points $\left(i, j_{0}, k_{i, j_{0}}\right),\left(i, j_{0}+\right.$ $\left.1, k_{i, j_{0}+1}\right), \cdots,\left(i, j_{1}, k_{i, j_{1}}\right)$ for $i \in[1, r]$.

To make the definition more transparent, let us translate it in the language of the pictorial representation I above. Each matrix $N_{i, j}$ corresponds to a lozenge made of two triangles (one grey, one white) sharing the horizontal edge $\left(i, j-1, k_{i, j-1}\right)-\left(i, j, k_{i, j}\right)$. The grey triangle is above ( $U$ matrix) if $k_{i, j}=k_{i, j-1}+1$, below ( $V$ matrix) if $k_{i, j}=$ $k_{i, j-1}-1$. Moreover, the order in which the $\left\{N_{i, j}\right\}_{i \in[1, r]}$ are multiplied to form the "slice" network matrix $N(j-1, j)$ exactly corresponds to a choice of diagonal in each square $\left(i-1, j-1, k_{i-1, j-1}\right)-\left(i-1, j, k_{i-1, j}\right)-\left(i, j-1, k_{i, j-1}\right)-\left(i, j, k_{i, j}\right)$, for $i=2,3, \ldots, r$, with the rule that the diagonal should connect two opposite vertices with the same value of $k$. This gives rise to six possible vertical configurations of two lozenges:
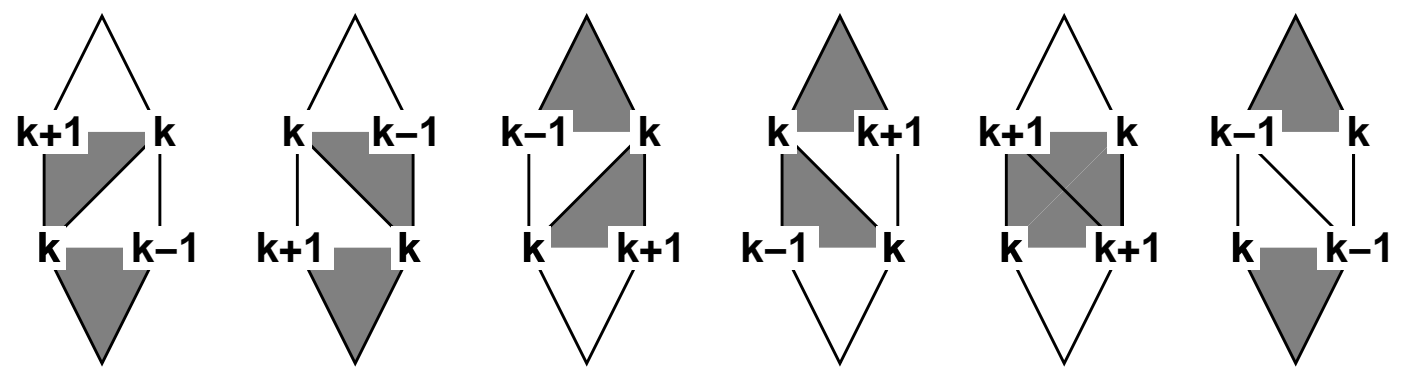

Note that when both pairs of diagonally opposite vertices have the same value of $k$, the choice of diagonal is not fixed. This ambiguity is immaterial, due to the identities (2.7) and (2.8). We have chosen the NW-SE diagonal by convention. We call this construction the $U, V$ decomposition of the stepped surface $\mathbf{k}$. 
Remark 2.1. There is a direct bijection between the $U, V$ decomposition of the stepped surfaces $\mathbf{k}$ and the quiver representing the B-matrix of the cluster algebra associated to the T-system [5]. Let $Q_{\mathbf{k}}$ be the (infinite) quiver encoded by the exchange matrix $B_{\mathbf{k}}$ at the node labeled by $\mathbf{k}$ in the cluster graph. We may represent $Q_{\mathbf{k}}$ with its vertices $(i, j)$ at the nodes of a square lattice $\mathbb{Z} \times \mathbb{Z}$ as a planar oriented graph with only square and triangular faces. Shading in grey the faces whose edges are oriented couterclockwise yields a tessellation with white and grey squares and triangles and corresponds to the $U, V$ decomposition of $\mathbf{k}$ described above. In particular, the six face configurations (2.15) correspond to the six following local quiver configurations:
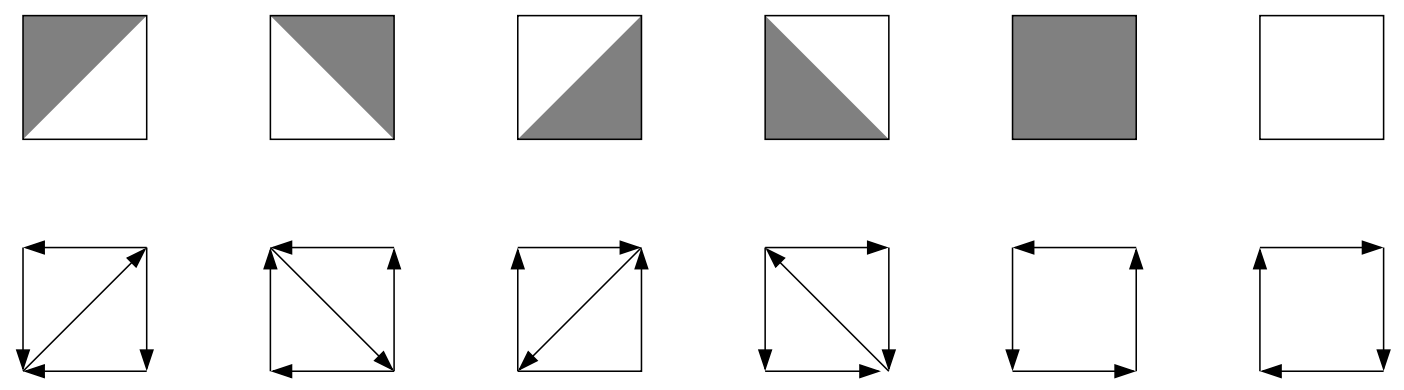

Remark 2.2. For notational simplicity the rules (2.15) are to be understood as seen from behind the initial data surface (i.e. from the opposite side of the surface to where the point $(i, j, k)$ lies), namely from an observer sitting at a point $\left(i, j, k^{\prime}\right)$ with $k^{\prime}<k_{i, j}$. This allows to read expressions such as products of $U, V$ matrices from left to right.

In the particular case of the fundamental stepped surface $\mathbf{k}_{0}$, as $k_{i, j}^{(0)} \in\{0,1\}$, the network matrices simplify to

$$
\begin{aligned}
N_{i, j} & =\left\{\begin{array}{ll}
U_{i}\left(t_{i, j-1}, t_{i, j}, t_{i+1, j-1}\right), & \text { if } i+j=0 \bmod 2 ; \\
V_{i}\left(t_{i-1, j}, t_{i, j-1}, t_{i, j}\right) & \text { otherwise. }
\end{array} \quad(i \in[1, r] ; j \in \mathbb{Z})\right. \\
N_{j} & =\prod_{i=1}^{r} N_{i, j}, \\
N\left(j_{0}, j_{1}\right) & =\prod_{j=j_{0}+1}^{j_{1}} N_{j}, \quad\left(j_{0} \leqslant j_{1}\right),
\end{aligned}
$$

still with the convention that $t_{0, j}=t_{r+1, j}=1$ and $N(j, j)=\mathbb{I}$.

The matrix $N\left(j, j^{\prime}\right)$ for the fundamental stepped surface $\mathbf{k}_{0}$ (we choose odd $j<j^{\prime} \in \mathbb{Z}$ 
in this example) is represented as follows:

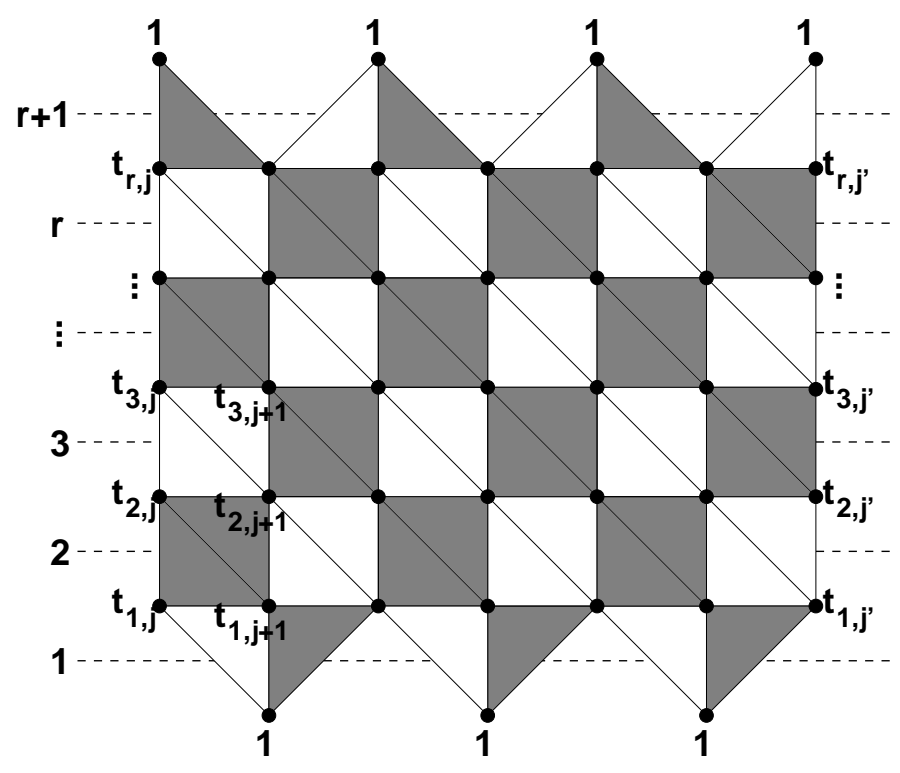

This corresponds to the following portion of the cluster algebra quiver (strictly speaking the bottom and top row of fixed values $T_{0, j, k}=T_{r+1, j, k}=1$ are not part of the cluster, and the corresponding nodes are not vertices of the quiver):

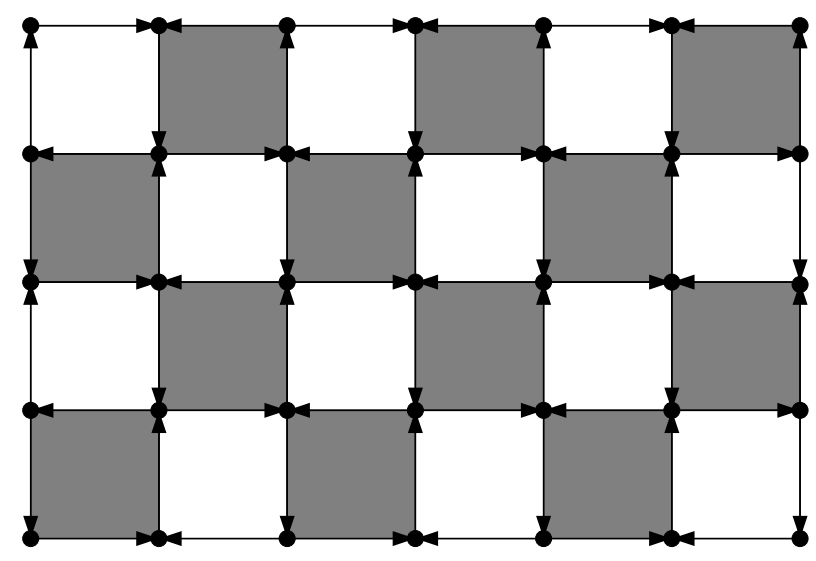


In the network picture, the matrix $N\left(j, j^{\prime}\right)$ is

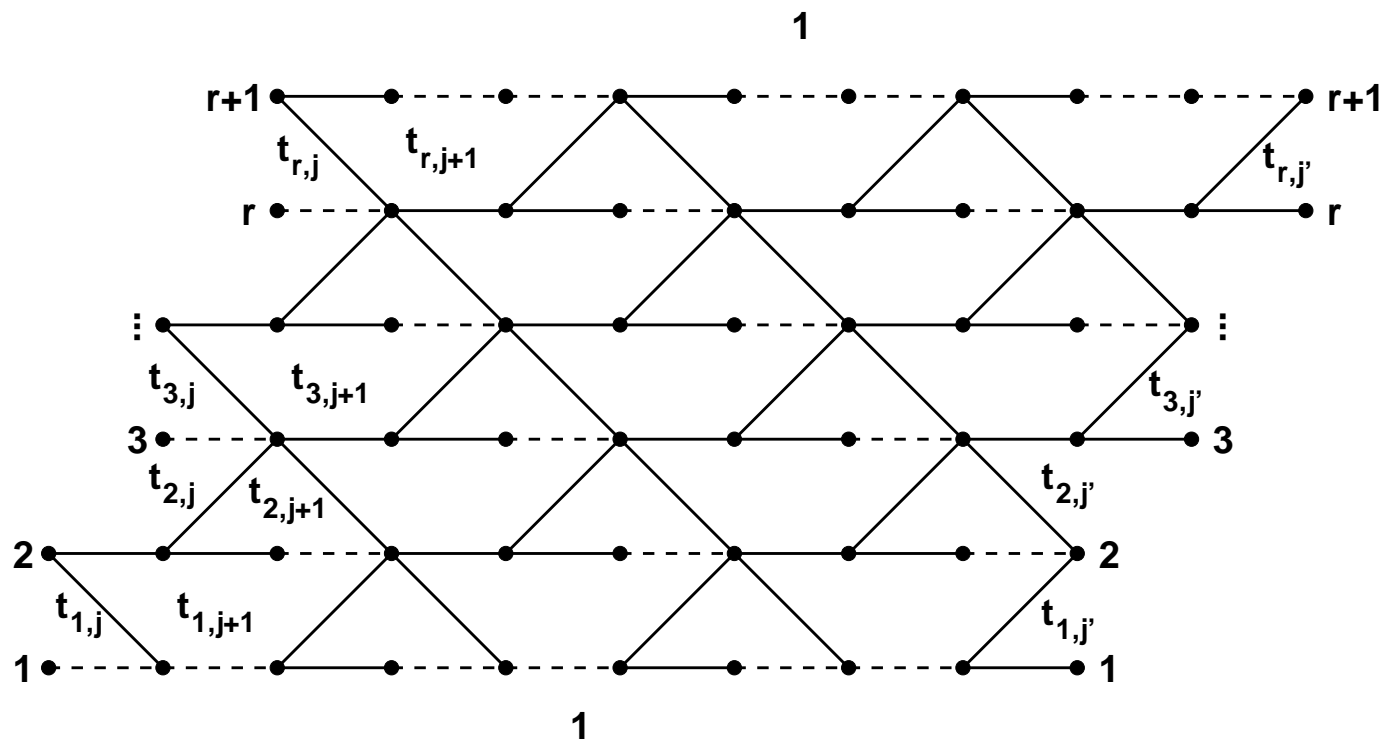

An example of a non-flat stepped surface $\mathbf{k}$, together with a pictorial representation of type I of its network matrix, is
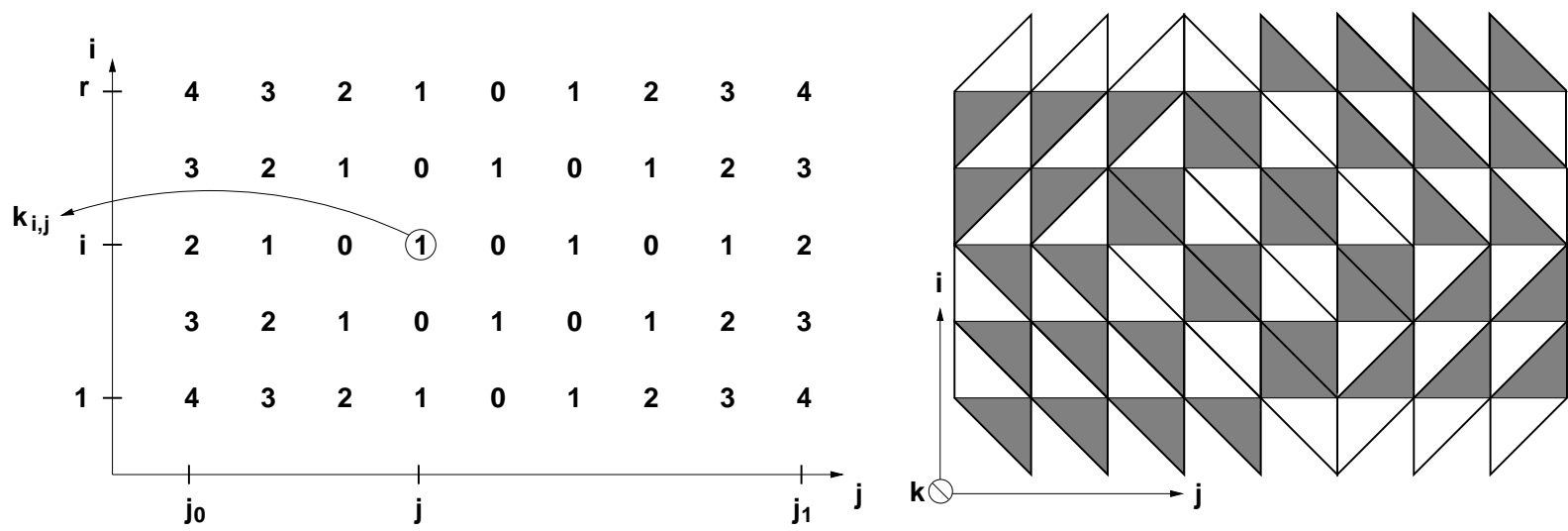

\section{$2.4 \quad A_{r} T$-system solution}

Following [4], we can now write an explicit expression for the variable $T_{1, j, k}$ in terms of the initial conditions $X_{\mathbf{k}}$ for any stepped surface $\mathbf{k}$. Without loss of generality, we may assume that the point $(1, j, k)$ is above $\mathbf{k}$, namely that $k \geqslant k_{1, j}$ (otherwise we simply reflect $k \rightarrow-k$ and $\mathbf{k} \rightarrow-\mathbf{k})$.

Definition 2.3. The projection of $(1, j, k)$ onto a given stepped surface $\mathbf{k}=\left\{\left(i, j, k_{i, j}\right)\right\}$ is the finite subset $\left\{\left(i, j, k_{i, j}\right)_{i \in[1, r] ; j \in\left[j_{0}, j_{1}\right]}\right\} \subset \mathbf{k}$. Here, $j_{0}$ is defined as largest integer such that

$$
k-j=k_{1, j_{0}}-j_{0},
$$

and $j_{1}$ defined as the smallest integer such that

$$
k+j=k_{1, j_{1}}+j_{1} .
$$


We call $j_{0}$ the minimum of the projection, and $j_{1}$ its maximum.

Theorem 2.4. [4] The solution $T_{1, j, k}$ of the $A_{r}$ T-system (1.1) in terms of the initial conditions $X_{\mathbf{k}}$ on a given stepped surface $\mathbf{k}$ is

$$
T_{1, j, k}=\left[N\left(j_{0}, j_{1}\right)\right]_{1,1} T_{1, j_{1}, k_{1, j_{1}}}
$$

For the proof, we refer the reader to [4].

The expression for $T_{i, j, k}$ with $i>1$ is obtained from the Wronskian expressions (1.8). Combinatorially, this determinant is easily interpreted via the Lindström-Gessel-Viennot theorem $[19,20]$ as the partition function of a family of $i$ non-intersecting paths on the weighted network corresponding to $N\left(j_{0}, j_{1}\right)$, where $j_{0}$ and $j_{1}$ are respectively the smallest minimum and largest maximum of the projections of the $T_{1, j^{\prime}, k^{\prime}}$ involved in the discrete Wronskian expression (1.8), namely with $\left(j^{\prime}, k^{\prime}\right)=(j+a-b, k+a+b-i-1), a, b \in[1, i]$.

Theorem 2.5. [4] As a function of $X_{\mathbf{k}}$, the solution $T_{i, j, k}$ of the $A_{r} T$-system is

$$
T_{i, j, k}=Z_{j_{0}(1), \ldots, j_{0}(i)}^{j_{1}(1), \ldots, j_{1}(i)}\left(j_{0}, j_{1}\right) \prod_{a=1}^{i} T_{1, j_{1}(a), k_{1, j_{1}(a)}}
$$

where $Z_{j_{0}(1), \ldots, j_{0}(i)}^{j_{1}(1), \ldots, j_{1}(i)}\left(j_{0}, j_{1}\right)$ is the partition function of $i$ non-intersecting weighted paths on the network corresponding to $N\left(j_{0}, j_{1}\right)$, starting at the points $\left(1, j_{0}(1)\right),\left(1, j_{0}(2)\right), \ldots$, $\left(1, j_{0}(i)\right)$ and ending at the points $\left(1, j_{1}(1)\right),\left(1, j_{1}(2)\right), \ldots,\left(1, j_{1}(i)\right)$. These points are respectively the minima of the projections of the points $(1, j+b-i, k-b), b=1,2, \ldots, i$, with coordinates $\left(1, j_{0}(b), k_{1, j_{0}(b)}\right)$, onto $\mathbf{k}$, and the maxima of the projections of the points $(1, j+a-1, k+a-i), a=1,2, \ldots, i$ onto $\mathbf{k}$, with coordinates $\left(1, j_{1}(a), k_{1, j_{1}(a)}\right)$.

\section{$3 \quad$ Unrestricted $A_{\infty} T$-system}

In this section, we study the solutions $T_{i, j, k}$ of the octahedron equation or the unrestricted $A_{\infty} T$-system (1.1), not subject to the restriction (1.6). The idea is that for given $i, j, k$, the solutions of the $A_{\infty}$ system are given by those of some $A_{r}$ system for sufficiently large $r$.

We wish to write the solution explicitly in terms of initial conditions $X_{\mathbf{k}}$ (1.3) specified along some stepped surface $\mathbf{k}(1.2)$.

As a preliminary remark, we note that the substitutions $k \rightarrow-k$ as well as $(i, j, k) \rightarrow$ $(i+a, j+b, k+c)$ for any $a, b, c \in \mathbb{Z}$ with $a+b+c$ even leave the $T$-system equation (1.1) invariant:

Lemma 3.1. The solution $T_{i, j, k}$ of the unrestricted $A_{\infty}$ T-system (1.1) with initial conditions $X_{\mathbf{k}}$ is the same function of the initial values $\left\{t_{x, y}\right\}$ as $T_{i, j,-k}$ with initial condition $X_{-\mathbf{k}}$, where by $-\mathbf{k}$ we mean the stepped surface $\mathbf{k}^{\prime}$ with $k_{i, j}^{\prime}=-k_{i, j}$ for all $i, j$. 
Lemma 3.2. The unrestricted $A_{\infty}$ T-system solution $T_{i, j, k}$ with initial conditions $X_{\mathbf{k}}$ is the same function of the initial values $t_{x, y}$ as $T_{i+a, j+b, k+c}$ is of the initial values $u_{x, y}=t_{x-a, y-b}$ for the initial conditions $X_{c+\mathbf{k}}$, for any $a, b, c \in \mathbb{Z}$, such that $a+b+c=0 \bmod 2$, and where by $c+\mathbf{k}$ we mean the stepped surface $\mathbf{k}^{\prime}$ with $k_{i, j}^{\prime}=k_{i, j}+c$ for all $i, j$.

As an immediate consequence of Lemmas 3.1 and 3.2, we may assume without loss of generality that the point $(i, j, k)$ is "above" $\mathbf{k}$, that is, $k \geqslant k_{i, j}$, as all the results for $k \geqslant k_{i, j}$ may be transferred to the case $k<k_{i, j}$ as well.

Definition 3.3. Let $\mathcal{D}_{\mathbf{k}}(i, j, k)=\left\{\left(x, y, k_{x, y}\right) \in \mathbb{Z}^{3}:|x-i|+|y-j| \leqslant\left|k-k_{x, y}\right|\right\} \subset \mathbf{k}$. $W e$ call $\mathcal{D}_{\mathbf{k}}(i, j, k)$ the shadow of the point $(i, j, k)$ on the initial data stepped surface $\mathbf{k}$.

Note that for $\mathbf{k}=\mathbf{k}_{0}$, the boundary points $\partial \mathcal{D}_{\mathbf{k}_{0}}(i, j, k)=\left\{\left(x, y, k_{x, y}^{(0)}\right):|x-i|+\right.$ $|y-j|=k-1\}$ all have $k_{x, y}^{(0)}=1$. For later purposes, we also define the interior domain $\mathcal{D}_{\mathbf{k}}^{\prime}(i, j, k)=\left\{\left(x, y, k_{x, y}\right):|x-i|+|y-j|<\left|k-k_{x, y}\right|\right\}$.

\subsection{Solution for the fundamental stepped surface $\mathrm{k}_{0}$}

We start with the case when $\mathbf{k}=\mathbf{k}_{0}$. Let us consider a point $(i, j, k)$ with $i+j+k=0$ $\bmod 2$, so that $k$ and $k_{i, j}^{(0)} \in\{0,1\}$ have the same parity. The following statement is clear from the form of the octahedron equation:

Lemma 3.4. The solution $T_{i, j, k}$ of the unrestricted $A_{\infty}$ T-system (1.1) with initial conditions $X_{\mathbf{k}_{0}}$ depends only on the initial values $t_{x, y}$ associated with points $\left(x, y, k_{x, y}^{(0)}\right) \in$ $\mathcal{D}_{\mathbf{k}_{0}}(i, j, k)$.

Lemma 3.2 has the following immediate consequence:

Lemma 3.5. The unrestricted $A_{\infty}$ T-system solution $T_{i, j, k}$ is the same function of the initial values $t_{x, y}$ on $\mathcal{D}_{\mathbf{k}_{0}}(i, j, k)$ as $T_{i+a, j+b, k+c}$ is of $u_{x, y}=t_{x-a, y-b}$ on $\mathcal{D}_{c+\mathbf{k}_{0}}(i+a, j+$ $b, c+k)$, for any $a, b, c \in \mathbb{Z}$, such that $a+b+c=0 \bmod 2$.

In view of the above Lemmas, we may immerse the domain $\mathcal{D}_{\mathbf{k}_{0}}(i, j, k)$ of initial data surface into a different initial data surface, pertaining to the $A_{r}$ case with sufficiently large $r$, so that the domain does not feel the $A_{r}$ boundary. More precisely, using the above-mentioned translational invariance, we have the following.

Lemma 3.6. The solution $T_{i, j, k}\left(\left\{t_{x, y}\right\}\right)$ of the $A_{\infty}$ T-system in terms of the initial values $t_{x, y}$ on $\mathcal{D}_{\mathbf{k}_{0}}(i, j, k)$ coincides with the solution $T_{k, 0, k}\left(\left\{u_{x, y}\right\}\right)$ of the $A_{r} T$-system, with $r=$ $2 k-1$, and with initial data $u_{x, y}$ on any stepped surface $\mathbf{k}$ such that $k_{x, y}=x+y \bmod 2$ for $\left(x, y, k_{x, y}\right) \in \mathcal{D}_{\mathbf{k}_{0}}(k, 0, k)$, on which $u_{x, y}=t_{x+i-k, y+j}$.

Proof. We use Lemma 3.4 to compare the solution $T_{i, j, k}\left(\left\{t_{x, y}\right\}\right)$ of the $A_{\infty} T$-system to that, $T_{k, 0, k}\left(\left\{u_{x, y}\right\}\right)$ of the $A_{r} T$-system with $r=2 k-1$. The latter only depends on the values $u_{x, y}$ on the shadow of $(k, 0, k)$ onto the stepped surface $\mathbf{k}$, which was engineered to be $\mathcal{D}_{\mathbf{k}_{0}}(k, 0, k)$. The lemma then follows from the translational invariance of Lemma 3.5, with $a=k-i, b=-j$ and $c=0$. 
To compute the solution $T_{i, j, k}$ of the unrestricted $A_{\infty} T$-system, we simply have to compute the solution $T_{k, 0, k}\left(\left\{u_{x, y}\right\}\right)$ of the $A_{2 k-1} T$-system.

Definition 3.7. The network matrix associated to the domain $\mathcal{D}_{\mathbf{k}_{0}}(k, 0, k)$, denoted by $N\left(\mathcal{D}_{\mathbf{k}_{0}}(k, 0, k)\right)$ is the product of the $2 k-2 \times 2 k-2 U$ and $V$ matrices corresponding to the $U, V$ decomposition of the domain $\mathcal{D}_{\mathbf{k}_{0}}(k, 0, k)$, according to the rules of eq.(2.15).

Example 3.8. For $k=3$, we have in the pictorial representation I:

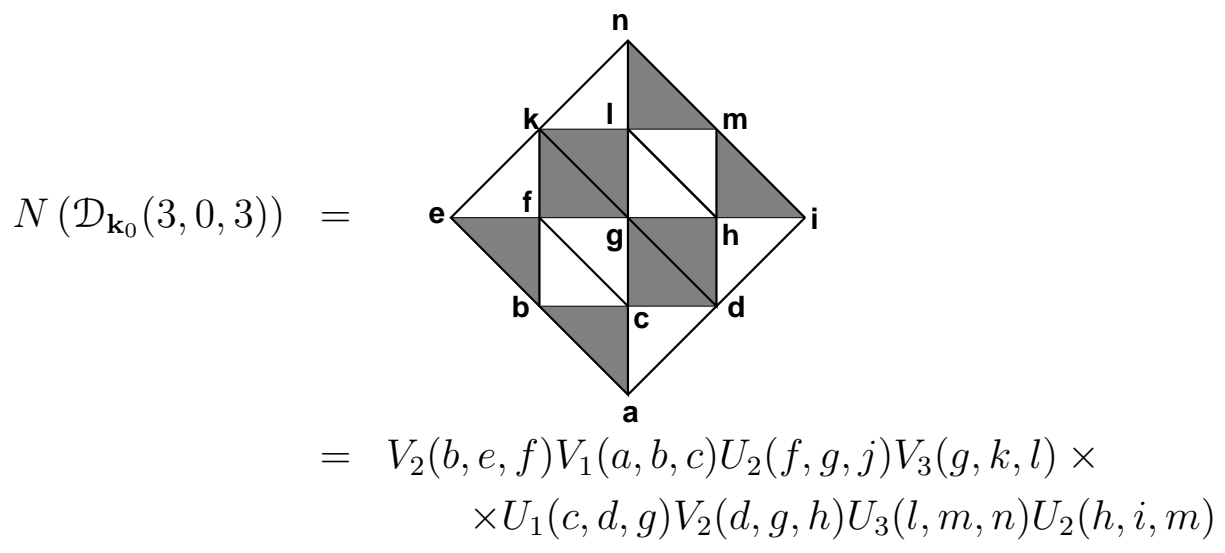

where we have used shorthands for the variables

$$
\begin{aligned}
& u_{5,0}=n \\
& u_{4,-1}=k \quad u_{4,0}=l \quad u_{4,1}=m \\
& u_{3,-2}=e \quad u_{3,-1}=f \quad u_{3,0}=g \quad u_{3,1}=h \quad u_{3,2}=i \\
& u_{2,-1}=b \quad u_{2,0}=c \quad u_{2,1}=d \\
& u_{1,0}=a
\end{aligned}
$$

We have the following.

Theorem 3.9. The solution $T_{k, 0, k}\left(\left\{u_{x, y}\right\}\right)$ of the $A_{2 k-1} T$-system is given by:

$$
T_{k, 0, k}=\left|N\left(\mathcal{D}_{\mathbf{k}_{0}}(k, 0, k)\right)_{1,2, \ldots, k-1}^{1,2, \ldots, k-1}\right| \prod_{a=1}^{k-1} u_{a, 1-a}^{-1} \prod_{b=1}^{k} u_{b, b-1}
$$

where for any matrix $M$ the notation $\left|M_{1,2, \ldots, m}^{1,2, \ldots, m}\right|$ stands for the $m \times m$ principal minor of $M$.

Proof. We apply Theorem 2.5 to the case of the following particular stepped surface $\mathbf{k}$. We assume that $\mathbf{k}$ satisfies the conditions of Lemma 3.6, and that, moreover, outside of $\mathcal{D}^{\prime} \mathbf{k}_{0}(k, 0, k), k_{x, y}$ is a strictly increasing function of $y$ for $y \geqslant 0$ and strictly decreasing for $y \leqslant 0$, while $u_{x, y}$ is arbitrary outside of $\mathcal{D}_{\mathbf{k}_{0}}(k, 0, k)$. As $\mathbf{k}$ and $\mathbf{k}_{0}$ coincide along $\mathcal{D}_{\mathbf{k}_{0}}(k, 0, k)$ we have:

$$
T_{k, 0, k}\left(\left\{u_{x, y}\right\}\right)=Z_{j_{0}(1), \ldots, j_{0}(k)}^{j_{1}(1), \ldots, j_{1}(k)}\left(j_{0}, j_{1}\right) \prod_{a=1}^{k} u_{1, j_{1}(a)}
$$


where we have used the initial data $T_{1, j_{1}(a), k_{1, j_{1}(a)}}=u_{1, j_{1}(a)}$, and

$$
\left\{\begin{array}{cc}
j_{0}(a)=a-k & j_{1}(a)=a-1 \\
k_{1, j_{0}(a)}=a-k+1 & k_{1, j_{1}(a)}=a
\end{array} \quad(a=1,2, \ldots, k)\right.
$$

with $j_{0}=1-k$ and $j_{1}=k-1$. The relevant part of the network involved in the quantity $Z_{j_{0}(1), \ldots, j_{0}(k)}^{j_{1}(1), \ldots, j_{1}(k)}\left(j_{0}, j_{1}\right)$ is the rectangle corresponding to $N\left(j_{0}, j_{1}\right)$, which reads in pictorial representations I and II:

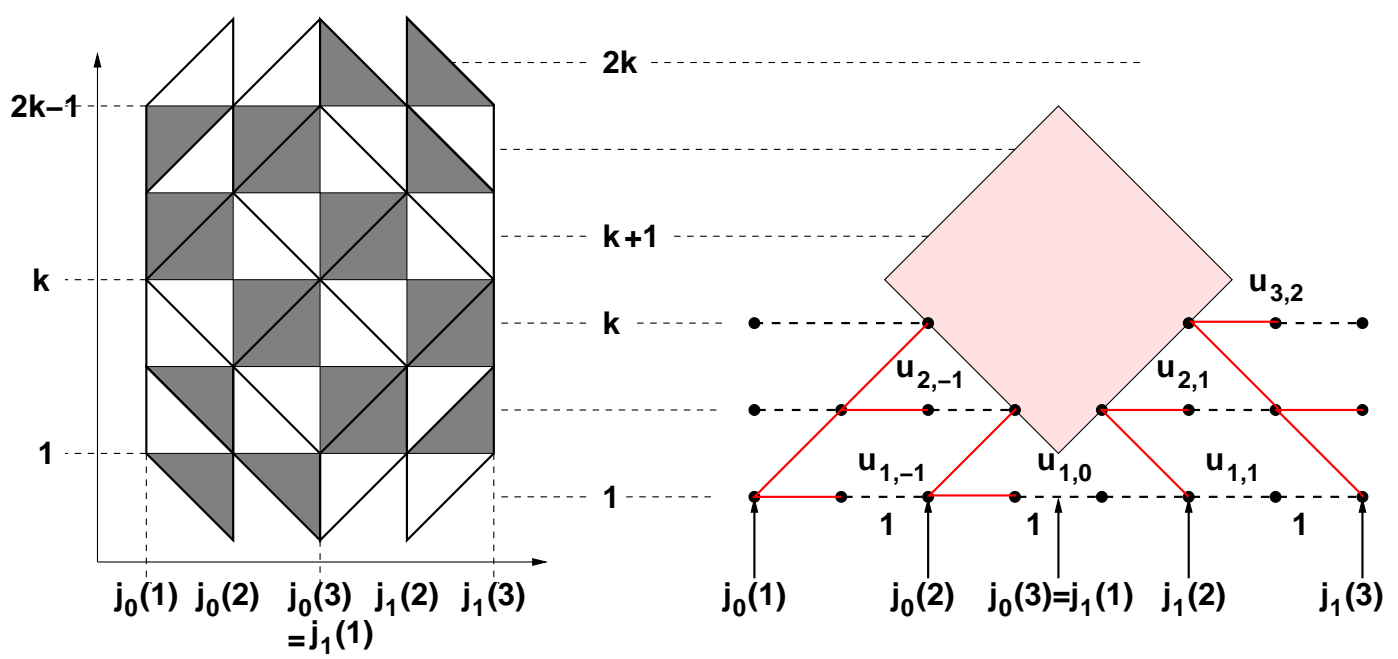

where in the second picture we have represented the network for $N\left(\mathcal{D}_{\mathbf{k}_{0}}(k, 0, k)\right)$ as a big lozenge with entry and exit points determined by the unique configuration of nonintersecting paths outside of that domain, that start at $j_{0}(a)$ and end at $j_{1}(k+1-a)$, $a=1,2, \ldots, k$. The steps of these paths outside of $N\left(\mathcal{D}_{\mathbf{k}_{0}}(k, 0, k)\right)$ are all diagonal steps of the network (going up on the left side, and down on the right side). The contribution from the $\mathrm{SW} \rightarrow \mathrm{NE}$ steps (on the left side) reads:

$$
\prod_{a=2}^{k} \prod_{m=1}^{k+1-a} \frac{u_{m-1, j_{0}(a)}}{u_{m, j_{0}(a)}}=\prod_{a=2}^{k} \frac{1}{u_{k+1-a, j_{0}(a)}}
$$

while the $\mathrm{NW} \rightarrow \mathrm{SE}$ steps (right side) contribute:

$$
\prod_{a=1}^{k} \prod_{m=1}^{a} \frac{u_{m+1, j_{1}(a)}}{u_{m, j_{1}(a)}}=\prod_{a=1}^{k} \frac{u_{a, j_{1}(a)}}{u_{1, j_{1}(a)}}
$$

Collecting the weights and substituting them into (3.1), the theorem follows, as, by virtue of the Lindström-Gessel-Viennot theorem [19,20], the quantity $\left|N\left(\mathcal{D}_{\mathbf{k}_{0}}(k, 0, k)\right)_{1,2, \ldots, k-1}^{1,2, \ldots, k-1}\right|$ is the partition function for families of $k-1$ non-intersecting weighted paths on the network for the above big lozenge, starting at all $k-1$ points on the SW border, and ending at all $k-1$ points on the SE border. 
Example 3.10. We continue with the example $k=3$ of Ex.3.8. We have

$$
\left|D_{\mathbf{k}_{0}}(3,0,3)\right|_{1,2}^{1,2}=\left|\begin{array}{cc}
\frac{b d+a g}{c d} & \frac{a(g i+d m)}{d h i} \\
\frac{e g+b k}{d f} & \frac{c(g i+d m)(e g+b k)}{d f g h i}+\frac{b(g n+m k)}{g i l}
\end{array}\right|
$$

The formula of Theorem 3.9 gives:

$$
\begin{aligned}
T_{3,0,3} & =\left|D_{\mathbf{k}_{0}}(3,0,3)\right|_{1,2}^{1,2} \frac{a d i}{a b} \\
& =\frac{b e g}{d f h}+\frac{b e m}{f h i}+\frac{b^{2} k}{d f h}+\frac{b^{2} m k}{f g h i}+\frac{b^{2} n}{c i l}+\frac{a b g n}{c d i l}+\frac{a b m k}{c d i l}+\frac{b^{2} m k}{c g i l}
\end{aligned}
$$

Theorem 3.9 has the following immediate consequence.

Corollary 3.11. The solution $T_{i, j, k}$ of the unrestricted $A_{\infty}$ T-system with initial conditions $X_{\mathbf{k}_{0}}$ is given by:

$$
T_{i, j, k}=\left|N\left(\mathcal{D}_{\mathbf{k}_{0}}(i, j, k)\right)_{1,2, \ldots, k-1}^{1,2, \ldots, k-1}\right| \prod_{a=1}^{k-2} t_{i+a-k+1, j-a}^{-1} \prod_{b=1}^{k-1} t_{i+b-k+1, j+b}
$$

\subsection{Solution for an arbitrary stepped surface $\mathrm{k}$}

Recall that $\mathcal{D}_{\mathbf{k}}(i, j, k)$ denotes the shadow of $(i, j, k)$ on $\mathbf{k}$, defined as the intersection of $\mathbf{k}$ with the pyramid $\Pi(i, j, k)=\{(x, y, z)|| x-i|+| y-j|\leqslant| z-k \mid\}$. Using Lemma 3.5, we may assume without loss of generality that $\mathcal{D}_{\mathbf{k}}(i, j, k)$ is entirely above $\mathbf{k}_{0}$. Indeed, $T_{i, j, m+k}$ is the same function of the initial data on $m+\mathbf{k}$ as $T_{i, j, k}$ on $\mathbf{k}$, so we may pick $m$ large enough to ensure that $m+k_{i, j} \geqslant k_{i, j}^{(0)}$ on $\mathcal{D}_{m+\mathbf{k}}(i, j, m+k)$.

As explained before, any finite domain of $\mathbf{k}$ above $\mathbf{k}_{0}$ may be obtained by applying a finite number of forward mutations $\mu_{i, j}$ to $\mathbf{k}_{0}$. These correspond to a local transformation of the surface, in which a vertex $\left(i, j, k_{i, j}=m-1\right)$ such that its four neighbors have $k_{i-1, j}=k_{i+1, j}=k_{i, j-1}=k_{i, j+1}=m$ is sent to the 6th vertex of the octahedron, $\left(i, j, k_{i, j}^{\prime}\right)$, with $k_{i, j}^{\prime}=m+1$, as illustrated in (1.5). If we complete $\mathcal{D}_{\mathbf{k}}(i, j, k)$ with the faces of $\Pi(i, j, k)$ until they intersect $\mathbf{k}_{0}$, we obtain a domain $\Delta_{\mathbf{k}}(i, j, k)$ that touches $\mathbf{k}_{0}$ along the square $|x-i|+|y-j|=k-1$. The domain $\Delta_{\mathbf{k}}(i, j, k)$ is obtained from $\mathcal{D}_{\mathbf{k}_{0}}(i, j, k)$ by a finite number of forward mutations of the form $\mu_{x, y}$ with $\left(x, y, k_{x, y}^{(0)}\right)$ strictly inside $\mathcal{D}_{\mathbf{k}_{0}}(i, j, k)$.

Starting from the expression of Corollary 3.11, we may implement these mutations by the corresponding $V U \leftrightarrow U V$ substitutions according to (2.5), as depicted in (2.9). These mutations are directly applied on the matrix $N\left(\mathcal{D}_{\mathbf{k}_{0}}(i, j, k)\right)$, until the matrix is expressed as $N\left(\Delta_{\mathbf{k}}(i, j, k)\right)$. We have consequently:

$$
T_{i, j, k}=\left|N\left(\Delta_{\mathbf{k}}(i, j, k)\right)_{1,2, \ldots, k-1}^{1,2, \ldots, k-1}\right| \prod_{a=1}^{k-2} t_{i+a-k+1, j-a}^{-1} \prod_{b=1}^{k-1} t_{i+b-k+1, j+b}
$$


We are left with the simple task of comparing $N\left(\Delta_{\mathbf{k}}(i, j, k)\right)$ with $N\left(\mathcal{D}_{\mathbf{k}}(i, j, k)\right)$. Let us denote by $L_{a}=\left(i_{a}, j_{a}\right)$ and $R_{a}=\left(i_{a}^{\prime}, j_{a}^{\prime}\right), a=1,2, \ldots, \kappa$, the $(i, j)$ coordinates of the vertices of $\partial \mathcal{D}_{\mathbf{k}}(i, j, k) \cap \Pi(i, j, k)$ with $\left(i_{a}, j_{a}\right)$ in the bottom left corner $i_{a} \leqslant i, j_{a} \leqslant j$ and $\left(i_{a}^{\prime}, j_{a}^{\prime}\right)$ in the bottom right corner $i_{a}^{\prime} \leqslant i, j_{a}^{\prime} \geqslant j$, labeled from bottom to top. We have:

Theorem 3.12. The solution $T_{i, j, k}$ of the unrestricted $A_{\infty} T$-system with initial conditions $X_{\mathrm{k}}$ reads:

$$
T_{i, j, k}=\left|N\left(\mathcal{D}_{\mathbf{k}}(i, j, k)\right)_{1,2, \ldots, \kappa-1}^{1,2, \ldots, \kappa-1}\right| \prod_{a=1}^{\kappa-1} t_{L_{a}}^{-1} \prod_{b=1}^{\kappa} t_{R_{b}}
$$

Proof. As $\Delta_{\mathbf{k}}(i, j, k)$ is a completion of $\mathcal{D}_{\mathbf{k}}(i, j, k)$ by use of the four faces of the pyramid $\Pi(i, j, k)$ until they reach $\mathbf{k}_{0}$, we have a simple pattern for the associated networks. Here is an example, with $(i, j, k=4)$ and its shadow $\mathcal{D}_{\mathbf{k}}(i, j, k)$ (shaded area) and domain $\Delta_{\mathbf{k}}(i, j, k)$ (within the dashed square) for a typical stepped surface whose heights $k_{i, j}$ are displayed on the left diagram:
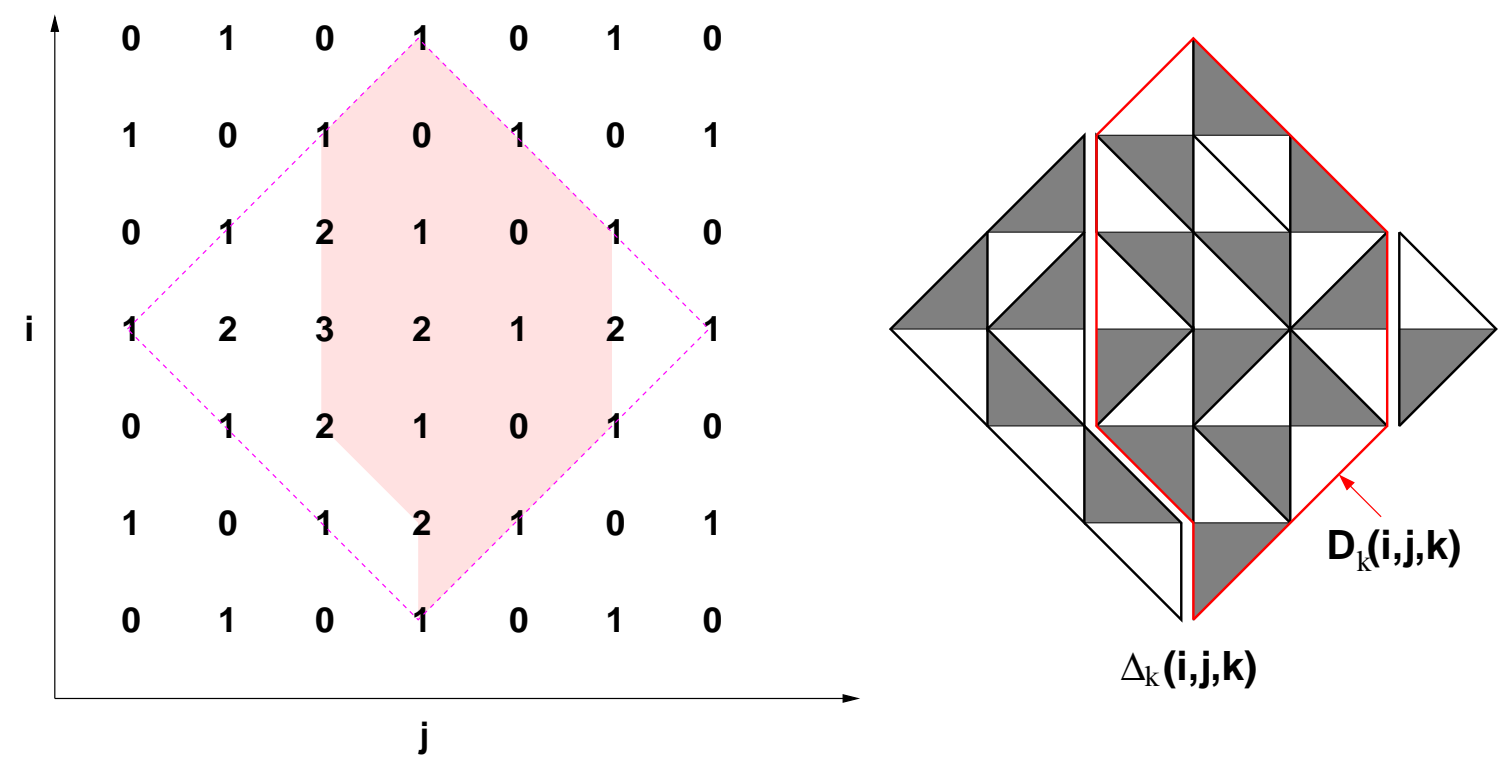

We have shown the corresponding matrix $N\left(\Delta_{\mathbf{k}}(i, j, k)\right)$ on the right, while $N\left(\mathcal{D}_{\mathbf{k}}(i, j, k)\right)$ corresponds to the smaller indicated domain, which matches the shaded domain on the left. Note that by construction the four corners between $\mathcal{D}_{\mathbf{k}}(i, j, k)$ and $\Delta_{\mathbf{k}}(i, j, k)$ are products of only $U$ 's (W corners) or only $V$ 's (E corners). The network pictorial repre- 
sentation is:


The vertex labels correspond to the actual initial data values, with $t_{L_{1}}=t_{R_{1}}=x, t_{L_{2}}=u^{\prime}$, $t_{L_{3}}=v^{\prime}, t_{R_{2}}=y, t_{R_{3}}=z, t_{R_{4}}=w^{\prime}$, while $\kappa=4$. We see that the (non-intersecting) paths contributing to $N\left(\Delta_{\mathbf{k}}(i, j, k)\right)$ must go along horizontal edges throughout the domain $\Delta_{\mathbf{k}}(i, j, k) \backslash \mathcal{D}_{\mathbf{k}}(i, j, k)$, as they correspond to $U$ matrices (W side) and $V$ matrices (E side). It is now easy to express $\left|N\left(\Delta_{\mathbf{k}}(i, j, k)\right)_{1,2, \ldots, k-1}^{1,2, \ldots, k-1}\right|$ in terms of $\left|N\left(\mathcal{D}_{\mathbf{k}}(i, j, k)\right)_{1,2, \ldots, \kappa-1}^{1,2, \ldots, \kappa-1}\right|$. Collecting the contributions of the horizontal steps of these paths, in the form of ratios of face labels along horizontals, all intermediate terms cancel out, leaving us with only the first and last one. In the particular example above, the weights of the horizontal steps transform the prefactor for the $\mathrm{W}$ side: $\frac{1}{u v x}$ into $\frac{u}{u^{\prime}} \frac{v}{v^{\prime}} \frac{1}{u v x}=\frac{1}{u^{\prime} v^{\prime} x}$, while on the E side we have: $x y z w$ transformed into $\frac{w^{\prime}}{w} x y z w=x y z w^{\prime}$. In general, the net result is to replace the factors of $t^{-1}$ 's and $t^{\prime}$ 's in (3.3) by the products of $t_{L_{a}}^{-1}$ and $t_{R_{a}}$, and the theorem follows.

Theorem 1.3 is now an immediate corollary of Theorem 3.12, as $N\left(\mathcal{D}_{\mathbf{k}}(i, j, k)\right)$ is the matrix of a network with edge weights that are non-negative Laurent monomials of the initial data $\left\{t_{i, j}\right\}$, and by the Lindström-Gessel-Viennot theorem $[19,20]$ the minor $N\left(\mathcal{D}_{\mathbf{k}}(i, j, k)\right)_{1,2,2, \ldots, k-1}^{1,2, \ldots-1}$ is the partition function of families of $\kappa-1$ weighted nonintersecting paths on the network graph, which is a polynomial of the path weights with non-negative integer coefficients.

Remark 3.13. A last remark is in order. In this section, we have used the known solution of the $A_{r}$ T-system (Theorem 2.5) to derive the general formula of Theorem 3.12 for the unrestricted $A_{\infty}$ T-system solutions. We may now reverse the logic and extend the formula (3.4) to the case of the $A_{r} T$-system solutions, by viewing the $A_{r} T$-system as a restriction of the $A_{\infty}$ one obtained by impsing the extra $A_{r}$ boundary condition. This is easily done by noting that the $A_{r}$ boundary simply truncates the space $(i, j, k) \mathbb{Z}^{3}$ to the domain $(i, j, k) \in[1, r] \times \mathbb{Z}^{2}$. Accordingly, the initial data stepped surfaces $\mathbf{k}$ are truncated to lie in the same domain, while the shadow of any given point $(i, j, k)$ on $\mathbf{k}$ is similarly truncated to a domain $\mathcal{D}_{\mathbf{k}}(i, j, k)=\mathbf{k} \cap \Pi(i, j, k)$. The formula (3.4) remains valid with 

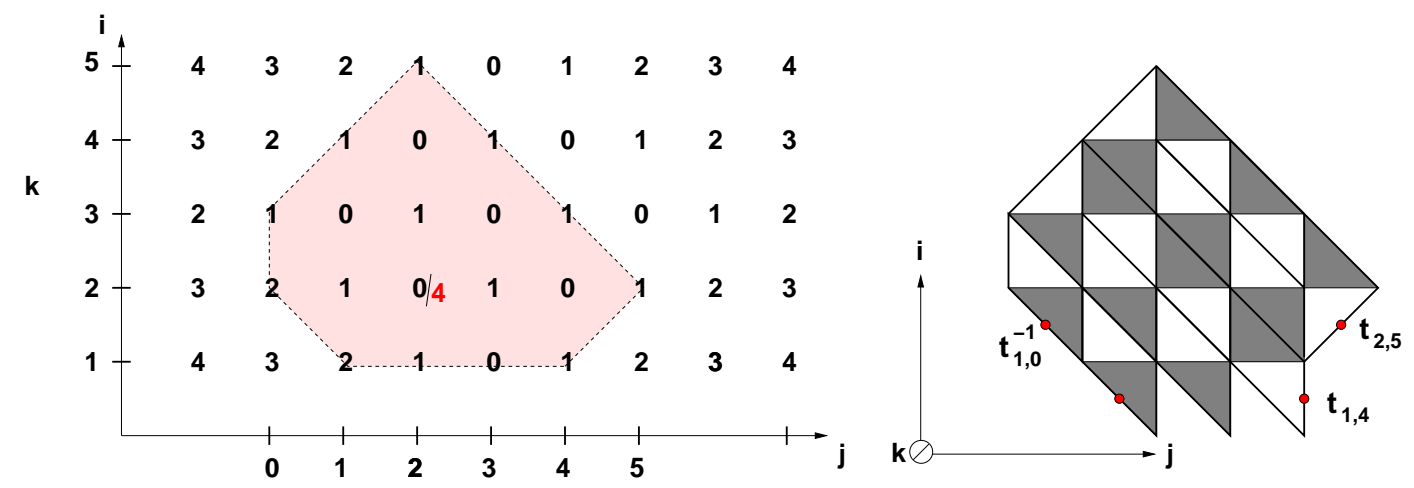

Figure 2: A typical application of the truncation of formula 3.4 in the $A_{r}$ case. We have $r=5,(i, j, k)=(2,2,4)$, and the surface $\mathbf{k}$ given on the left where we indicate the value of $k$ in the $(i, j)$ coordinate plane. The shaded area is the (truncated) shadow of $(i, j, k)$ on $\mathbf{k}$. The formula expresses $T_{i, j, k}$ as the partition function for $i=2$ non-intersecting paths on the lattice $N\left(\mathcal{D}_{\mathbf{k}}(i, j, k)\right)$ represented on the right, with the indicated prefactors.

this new definition, while the left and right boundary points range only over those within the truncated domain (see Fig. 2 for an example). This gives a new direct formula for the solution of the $A_{r} T$-system which displays manifest Laurent positivity of the solution in terms of arbitrary initial data.

\section{4 l-restricted T-system: the $A_{1}$ case}

We study the solutions of the $T$-system with $\ell$-restricted boundaries. For pedagogical reasons, this section is entirely devoted to the case of $A_{1}$, for which we will derive slightly more general results.

\subsection{The $\ell$-restricted $A_{1} T$-system and its initial conditions}

The $A_{1} T$-system is the $r=1$ version of (1.1), with the simplified notation $T_{1, j, k}=T_{j, k}$ for $j, k \in \mathbb{Z}$ and $j+k=1 \bmod 2$. Let $S$ be a subset of $\mathbb{Z}$. We consider the $A_{1} T$-system with the restriction that $j \in S$ :

$$
T_{j, k+1} T_{j, k-1}=T_{j+1, k} T_{j-1, k}+1 \quad(j \in S ; k \in \mathbb{Z})
$$

The general initial conditions for (4.1) are indexed by stepped surfaces $\mathbf{k}$ (1.2), which reduce here to paths

$$
\mathbf{k}(S)=\left\{k_{j}(S) \in \mathbb{Z},\left|k_{j+1}-k_{j}\right|=1 \text { and } k_{j}+j=1 \bmod 2, j \in S\right\} .
$$

We consider the system (4.1) with possibly additional boundary conditions depending on $S$, and an initial condition $X^{S}(\mathbf{t})$, which is an assignment of formal variables $\mathbf{t}=\left(t_{j}\right)_{j \in S}$ to the points on the surface $\mathbf{k}(S)$. We consider the four cases: 
(i) Unrestricted $A_{1} T$-system: $S=\mathbb{Z}$. The initial condition $X_{\mathbf{k}}(\mathbf{t})$ is the assignment

$$
X_{\mathbf{k}}(\mathbf{t}):\left\{T_{j, k_{j}}=t_{j}(j \in \mathbb{Z})\right\}
$$

(ii) Right half-plane $A_{1} T$-system: $S=[1, \infty)$. The additional boundary conditions are

$$
T_{0, k}=1 \quad(k \in \mathbb{Z})
$$

and the initial condition $X_{\mathbf{k}}^{+}(\mathbf{t})$ is the assignment

$$
X_{\mathbf{k}}^{+}(\mathbf{t}):\left\{T_{j, k_{j}}=t_{j}(j \in[1, \infty))\right\}
$$

(iii) Left half-plane $A_{1} T$-system: $S=(-\infty, \ell]$. The additional boundary conditions are

$$
T_{\ell+1, k}=1 \quad(k \in \mathbb{Z})
$$

and the initial condition $X_{\mathbf{k}}^{-}(\mathbf{t})$ is the assignment

$$
X_{\mathbf{k}}^{-}(\mathbf{t}):\left\{T_{j, k_{j}}=t_{j}(j \in(-\infty, \ell])\right\}
$$

(iv) $\ell$-restricted $A_{1} T$-system: $S=[1, \ell]$. The additional boundary conditions are

$$
T_{0, k}=T_{\ell+1, k}=1 \quad(k \in \mathbb{Z})
$$

and the initial condition $X_{\mathbf{k}}^{[1, \ell]}(\mathbf{t})$ is the assignment

$$
X_{\mathbf{k}}^{[1, \ell]}(\mathbf{t}):\left\{T_{j, k_{j}}=t_{j}(j \in[1, \ell])\right\}
$$

In the following, we will also consider the unrestricted $A_{1} T$-system (i) with initial conditions $X_{\mathbf{k}}(\mathbf{t})$ (5.2), further restricted by imposing extra conditions on the initial values $\mathbf{t}=\left\{t_{j}\right\}$ as well as the path $\mathbf{k}$. The new initial values $\mathbf{t}^{+}, \mathbf{t}^{-}, \mathbf{t}^{[1, \ell]}$ and paths $\mathbf{k}^{+}, \mathbf{k}^{-}, \mathbf{k}^{[1, \ell]}$ correspond respectively to the following conditions:

$$
\begin{gathered}
\mathbf{t}^{+}:\left\{\begin{array}{c}
t_{-j-2}=-t_{i, j}\left(j \in \mathbb{Z}_{+}\right) \\
t_{0}=1, t_{-1}=0
\end{array} \quad \mathbf{k}^{+}:\left\{\begin{array}{c}
k_{-j-2}=k_{j}\left(j \in \mathbb{Z}_{+}\right) \\
k_{-1}=k_{0}-1
\end{array}\right.\right. \\
\mathbf{t}^{-}:\left\{\begin{array}{c}
t_{j+\ell+3}=-t_{\ell+1-j}\left(j \in \mathbb{Z}_{+}\right) \\
t_{i, \ell+1}=1, t_{i, \ell+2}=0
\end{array} \quad \mathbf{k}^{-}:\left\{\begin{array}{c}
k_{j+\ell+3}=k_{\ell+1-j}\left(j \in \mathbb{Z}_{+}\right) \\
k_{\ell+2}=k_{\ell+1}-1
\end{array}\right.\right. \\
\mathbf{t}^{[1, \ell]}:\left\{\begin{array}{c}
t_{-j-2}=-t_{i, j}\left(j \in \mathbb{Z}_{+}\right) \\
t_{2(\ell+3)+j}=t_{i, j}(j \in \mathbb{Z}) \\
t_{0}=t_{\ell+1}=1, t_{-1}=0
\end{array} \quad \mathbf{k}^{[1, \ell]}:\left\{\begin{array}{c}
k_{-j-2}=k_{j}\left(j \in \mathbb{Z}_{+}\right) \\
\left.k_{j+2(\ell+3}\right)=k_{j}(j \in \mathbb{Z}) \\
k_{-1}=k_{0}-1, k_{\ell+2}=k_{\ell+1}-1
\end{array}\right.\right.
\end{gathered}
$$

By convention, when $\mathbf{k}=\mathbf{k}_{0}$ we drop the requirements on $\mathbf{k}$. We note that the conditions (4.8) are equivalent to imposing simultaneously the conditions (4.6) and (4.7).

We wish to study the solutions $T_{j, k}$ of the $A_{1} T$-system in terms of initial conditions in all of the above cases $(i-i v)$. By virtue of Lemmas 3.1 and 3.2 we may without loss of generality restrict ourselves to points $(j, k)$ above the initial data surface $\mathbf{k}$ in all these cases. 


\subsection{Unrestricted system solution}

The unrestricted system subject to initial conditions $X_{\mathbf{k}}(\mathbf{t})$ reads:

$$
\begin{aligned}
T_{j, k+1} T_{j, k-1} & =T_{j+1, k} T_{j-1, k}+1 \\
T_{j, k_{j}} & =t_{j} \quad(j \in \mathbb{Z})
\end{aligned} \quad(j, k \in \mathbb{Z} ; j+k=1 \bmod 2)
$$

Its solution is simply expressed in terms of the following simplified versions of the $U, V$ matrices of Sect. 2.1 defined as:

$$
U(a, b)=U(a, b, 1)=\left(\begin{array}{cc}
1 & 0 \\
b^{-1} & a b^{-1}
\end{array}\right) \quad V(a, b)=V(1, a, b)=\left(\begin{array}{cc}
a b^{-1} & b^{-1} \\
0 & 1
\end{array}\right) .
$$

Let us consider a point $(j, k)$ above the path $\mathbf{k}$, i.e. with $k \geqslant k_{j}$. Def. 2.3 for $r=1$ defines the projection of the point $(j, k)$ onto $\mathbf{k}$ as the portion of the path $\left(j, k_{j}\right)_{j \in\left[j_{0}, j_{1}\right]}$ with largest $j_{0}$ and smallest $j_{1}$ such that $k-k_{j_{0}}=j-j_{0}, k-k_{j_{1}}=j_{1}-j$. Note that $j_{0}$ and $j_{1}$ are both even integers. The cone of projection of $(j, k)$ is defined by the two lines $k=j+k_{j_{0}}-j_{0}$ and $k=k_{j_{1}}+j_{1}-j$.

We define the matrix

$$
M_{j}\left(t_{j}, t_{j+1}\right)=\left\{\begin{array}{lll}
V\left(t_{j}, t_{j+1}\right) & \text { if } & k_{j}=k_{j+1}+1 \\
U\left(t_{j}, t_{j+1}\right) & \text { if } & k_{j}=k_{j+1}-1
\end{array}\right.
$$

We have:

Theorem 4.1. [1],[4] The solution $T_{j, k}$ of the system (4.9-4.10) is:

$$
T_{j, k}=\left(\prod_{j=j_{0}}^{j_{1}-1} M_{j, j+1}\left(t_{j}, t_{j+1}\right)\right)_{1,1} t_{j_{1}}
$$

Note that this is the $A_{1}$ version of (2.18), in which the $2 \times 2$ network matrix $N\left(j_{0}, j_{1}\right)$ is identified with the $2 \times 2$ matrix product $\prod_{j=j_{0}}^{j_{1}-1} M_{j, j+1}\left(t_{j}, t_{j+1}\right)$.

The exact solution of Theorem 4.1 was used previously to derive the positive Laurent property for the solution of the $T$-system, namely that $T_{j, k}$ is a Laurent polynomial of the initial data, with non-negative integer coefficients. (This is clear from Theorem 4.1, as the entries of $U, V$ are themselves Laurent monomials of the initial data with non-negative integer coefficients.).

\section{3 $\ell$-restricted case: equivalent initial data and main theorems}

We now turn to solutions of the $\ell$-restricted system. The main idea is to realize the $\ell$-restricted boundaries within the framework of the unrestricted $T$-system, by suitably engineering the initial data $t_{k}, k \in \mathbb{Z}$. The following three theorems will be proved in next section. 
Theorem 4.2. The solution $T_{j, k}$ of the $\ell$-restricted $A_{1}$ T-system (iv) is periodic in the direction $k$ :

$$
T_{j, k+N}=T_{j, k}
$$

with period $N=2(\ell+3)$.

Theorem 4.3. The solution of the unrestricted $A_{1} T$-system (i) with initial conditions $X_{\mathbf{k}_{0}}\left(\mathbf{t}^{[1, \ell]}\right)$ (4.2,4.8) restricts to the solution of the $\ell$-restricted $A_{1}$ T-system (iv) with the initial conditions $X_{\mathbf{m}}^{[1, \ell]}(\mathbf{u})(4.5)$, where $\mathbf{u}=\mathbf{t}([1, \ell]), \mathbf{m}=\mathbf{k}_{0}([1, \ell])$ are the restrictions of $\mathbf{t}, \mathbf{k}_{0}$ to the interval $j \in[1, \ell]$. As such, the solution of the $\ell$-restricted $A_{1}$ T-system with initial conditions $X_{\mathbf{k}_{0}}^{[1, \ell]}(\mathbf{t})$ is a positive Laurent polynomial of the initial values $t_{1}, t_{2}, \ldots, t_{\ell}$.

Theorem 4.4. The solution of the $\ell$-restricted $A_{1}$ T-system (iv) with initial conditions $X_{\mathbf{k}}(\mathbf{t})$ (4.5) along an arbitrary finite path $\mathbf{k}$ is a positive Laurent polynomial of the initial values $t_{1}, t_{2}, \ldots, t_{\ell}$.

\subsection{Half-plane solution}

To prove Theorem 4.3, we must show that the $\ell$-restricted boundary is implemented by the choice of symmetries of the initial data. Concretely, one must show that both $T_{0, k}=1$ and $T_{\ell+1, k}=1$ as a consequence. It turns out to be instructive to first consider the case of the $T$-system (4.1) in a half-plane. We have:

Theorem 4.5. The solution of the unrestricted $A_{1}$ T-system (i) with initial conditions $X_{\mathbf{k}_{0}}\left(\mathbf{t}^{+}\right)$(4.2,4.6) restricts to that of the right half-plane $A_{1}$ T-system (ii) with initial condition $X_{\mathbf{m}}^{+}(\mathbf{u})(4.3)$, where $\mathbf{u}=\mathbf{t}([1, \infty)), \mathbf{m}=\mathbf{k}_{0}([1, \infty))$ are the restrictions of $\mathbf{t}, \mathbf{k}_{0}$ to the range $j \in[1, \infty)$. As such the solutions of the latter are positive Laurent polynomials of the initial data $t_{1}, t_{2}, t_{3}, \ldots$

Proof. To prove the first statement of the theorem, it is sufficient to show that $T_{0,2 k+1}=1$ for all $k \geqslant 0$ (the case $k<0$ follows from the general reflection symmetry argument of Lemmas 3.1 and 3.2). Indeed, the half-plane solution is uniquely determined in terms of initial conditions of the type (4.3), so it must coincide with that of the unrestricted system in the range $j \geqslant 0$, once the boundary condition $T_{0, k}=1$ is guaranteed.

To compute $T_{0,2 k+1}$, we wish to use Theorem 4.1, but we cannot plug directly the value $t_{-1}=0$ as some entries of the matrices $U, V$ may diverge. However, only combinations of the form $V\left(t_{j}, t_{j+1}\right) U\left(t_{j+1} t_{j+2}\right)$ for even $j$ enters the solution (4.13). We simply note that

$$
\lim _{\epsilon \rightarrow 0} V(-1, \epsilon) U(\epsilon, 1)=P=\left(\begin{array}{ll}
0 & 1 \\
1 & 0
\end{array}\right)
$$

Provided we take this limit, we may now safely use the formula (4.13) for $T_{0,2 k+1}$, for $k \geqslant 0$ with $j_{0}=-2 k+2$ and $j_{1}=2 k$ :

$$
\begin{aligned}
& T_{0,2 k+1}= \\
& \quad\left(V\left(-t_{2 k-2},-t_{2 k-3}\right) \cdots V\left(-t_{2},-t_{1}\right) U\left(-t_{1},-1\right) P V\left(1, t_{1}\right) U\left(t_{1}, t_{2}\right) \cdots U\left(t_{2 k-1}, t_{2 k}\right)\right)_{1,1} t_{2 k}
\end{aligned}
$$




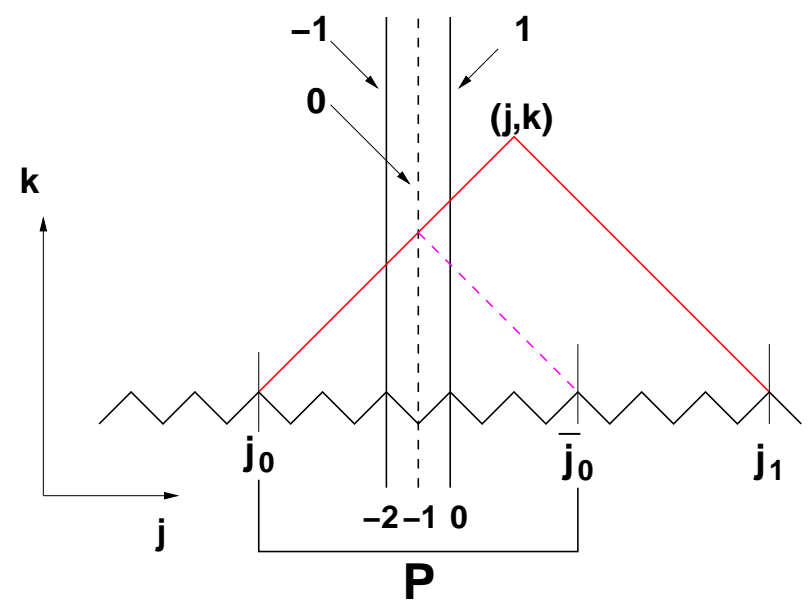

Figure 3: The segment $\left[j_{0}, j_{1}\right]$ of values necessary to express $T_{j, k}$ has its left endpoint reflected by the line $j=-1$. As a result, only the values of $t_{j}$ for $j$ between $\bar{j}_{0}=-j_{0}-2$ and $j_{1}$ enter the expression.

(Here and in the following the $\cdots$ stand for alternating products of $U V U V U \ldots$. . Next, we shall use the following "collapse" properties of $U, V, P$ matrices, easily derived by direct calculation:

$$
U(-b,-a) P V(a, b)=P \quad V(-b,-a) P U(a, b)=P
$$

for all $a, b$. Applying these iteratively to (4.13) implies:

$$
T_{0,2 k+1}=\left(P V\left(t_{2 k-2}, t_{2 k-1}\right) U\left(t_{2 k-1}, t_{2 k}\right)\right)_{1,1} t_{2 k}=\frac{1}{t_{2 k}} \times t_{2 k}=1
$$

We now turn to the Laurent positivity of the solution. Let us compute $T_{j, k}$ for $k \geqslant 0$ via (4.13). If $j_{0} \geqslant 0$, this is the same as the solution of the unrestricted system, and the positivity is clear. Otherwise, let us denote by $\bar{j}_{0}=-j_{0}-2 \geqslant 0$, and compute:

$$
\begin{aligned}
T_{j, k} & =\left(V\left(t_{j_{0}}, t_{j_{0}+1}\right) \cdots U\left(t_{-3},-1\right) P V\left(1, t_{1}\right) \cdots U\left(t_{j_{1}-1}, t_{j_{1}}\right)\right)_{1,1} t_{j_{1}} \\
& =\left(V\left(-t_{\bar{j}_{0}},-t_{\bar{j}_{0}-1}\right) \cdots U\left(-t_{1},-1\right) P V\left(1, t_{1}\right) \cdots U\left(t_{j_{1}-1}, t_{j_{1}}\right)\right)_{1,1} t_{j_{1}} \\
& =\left(P V\left(t_{\bar{j}_{0}}, t_{\bar{j}_{0}+1}\right) \cdots U\left(t_{j_{1}-1}, t_{j_{1}}\right)\right)_{1,1} t_{j_{1}}
\end{aligned}
$$

where we have used (4.15) repeatedly to eliminate the first $\bar{j}_{0}$ terms. This is a product of matrices with entries that are all Laurent monomials of the initial data $\left(t_{j}\right)_{j \geqslant 1}$ with non-negative integer coefficients. The positive Laurent property follows.

Remark 4.6. There is a very simple pictorial interpretation of the computation of $T_{j, k}$. The left endpoint of the segment $j \in\left[j_{0}, j_{1}\right]$ of initial values $t_{j}$ necessary to express the solution $T_{j, k}$ has been reflected by the line $j=-1$. This is depicted in Fig. 3, along with the corresponding cone of projection of $(j, k)$ and its reflection.

We have the following analogous result for the left half-plane $j \leqslant \ell$ solution: 
Theorem 4.7. For fixed $\ell \in \mathbb{Z}_{>0}$, the solution of the unrestricted $A_{1} T$-system (i) with initial conditions $X_{\mathbf{k}_{0}}\left(\mathbf{t}^{-}\right)(4.2,4.7)$, restricts to that of the left half-plane $A_{1} T$-system (iii) with initial condition $X_{\mathbf{m}}^{-}(\mathbf{u})(4.4)$, where $\mathbf{u}=\mathbf{t}((-\infty, \ell]), \mathbf{m}=\mathbf{k}_{0}((-\infty, \ell])$ are the restrictions of $\mathbf{t}, \mathbf{k}_{0}$ to the range $j \in(-\infty, \ell]$. As such the solutions of the latter are positive Laurent polynomials of the initial data $t_{\ell}, t_{\ell-1}, t_{\ell-2}, \ldots$

Proof. Let us first show the positivity statement. Imitating the proof of Theorem 4.5, we must "regularize" the singular value 0 by introducing:

$$
\lim _{\epsilon \rightarrow 0} V(1, \epsilon) U(\epsilon,-1)=-P
$$

A new feature arises when $\ell$ is even: in that case, the boundary contribution is

$$
\lim _{\epsilon \rightarrow 0} U(1, \epsilon) V(\epsilon,-1)=-P
$$

as well, but if $j+k=\ell+3$, the formula (4.13) for $T_{j, k}$ contains a potential singularity as $U(1, \epsilon)$ diverges when $\epsilon \rightarrow 0$. Fortunately the full formula also has an $\epsilon=T_{j_{1}, k_{j_{1}}}$ in factor, leading to a finite limit:

$$
\begin{aligned}
T_{j, k} & =\lim _{\epsilon \rightarrow 0}\left(V\left(t_{j-k}, t_{j-k+1}\right) \cdots V\left(t_{\ell}, 1\right) U(1, \epsilon)\right)_{1,1} \epsilon \\
& =\left(V\left(t_{j-k}, t_{j-k+1}\right) \cdots V\left(t_{\ell}, 1\right)\right)_{1,2}=\left(V\left(t_{j-k}, t_{j-k+1}\right) \cdots V\left(t_{\ell}, 1\right)(-P)\right)_{1,1}(-1)
\end{aligned}
$$

which is manifestly positive. In general, we compute $T_{j, k}$ via the formula (4.13). Again, if $j+k \leqslant \ell+1$, the solution is the same as in the unrestricted case, and positivity follows. Otherwise, we have a reflection of the segment of initial values on the right against the line $j=\ell+2$. More precisely, denoting by $M_{\ell}=U$ if $\ell$ is odd and $M_{\ell}=V$ if $\ell$ is even, we get:

$$
\begin{aligned}
T_{j, k} & =\left(V\left(t_{j-k}, t_{j-k+1}\right) \cdots M_{\ell}\left(t_{\ell}, t_{\ell+1}\right)(-P) M_{\ell+1}\left(t_{\ell+2}, t_{\ell+3}\right) \cdots U\left(t_{j+k-1}, t_{j+k}\right)\right)_{1,1} t_{j+k} \\
& =\left(V\left(t_{j-k}, t_{j-k+1}\right) \cdots U\left(t_{2(\ell+2)-j-k-1}, t_{2(\ell+2)-j-k}\right)(-P)\right)_{1,1}\left(-t_{2(\ell+2)-j-k}\right)
\end{aligned}
$$

where we have used the symmetry $t_{2(\ell+2-j)}=-t_{j}$ and (4.15) to cancel out terms on both sides of the $(-P)$ factor. The two minus signs cancel, and we are left with a manifestly positive expression. Let us now turn to the first part of the theorem. By uniqueness of the solution in the left half-plane, we simply have to show that $T_{\ell+1, k}=1$ for all $k \in \mathbb{Z}_{>0}$ such that $k+\ell$ is even. For odd $\ell$, using (4.16) we first compute: $T_{\ell+1,2}=\lim _{\epsilon \rightarrow 0}\left(V\left(t_{\ell}, 1\right) U(1, \epsilon)\right)_{1,1} \epsilon=1$. For all other cases, we use (4.17) to compute:

$$
\begin{aligned}
T_{\ell+1, k} & =\left(V\left(t_{\ell+1-k}, t_{\ell+2-k}\right) U\left(t_{\ell+2-k}, t_{\ell+3-k}\right) P\right)_{1,1} t_{\ell+3-k} \\
& =\left(V\left(t_{\ell+1-k}, t_{\ell+2-k}\right) U\left(t_{\ell+2-k}, t_{\ell+3-k}\right)\right)_{1,2} t_{\ell+3-k}=\frac{1}{t_{\ell+3-k}} \times t_{\ell+3-k}=1
\end{aligned}
$$

This completes the proof of the theorem. 


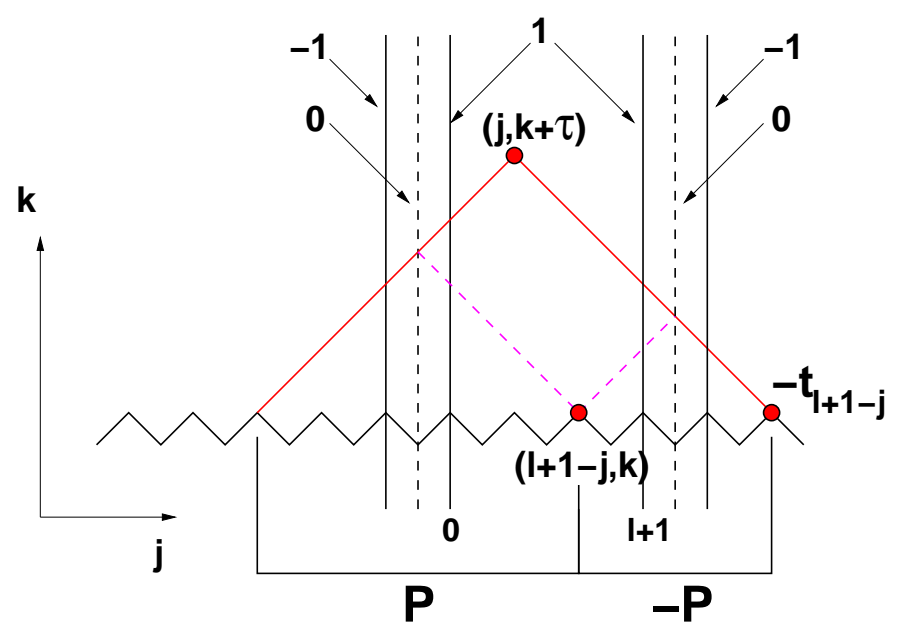

Figure 4: For odd $k$, the segment of values necessary to express $T_{j, k+N / 2}$ is reflected by both lines $j=-1$ and $j=\ell+2$ picking respectively a factor $P$ or $-P$ per reflection. After these two reflections, the edges of the cone of projection of $(j, k+N / 2)$ meet again at the point $(\ell+1-j, k)$. We have indicated the contributions from the formula (4.13).

\section{$4.5 \ell$-restricted boundaries and periodicity: proof of Theorem 4.2}

Combining Theorems 4.5 and 4.7, we immediately deduce the following:

Theorem 4.8. The solution of the $\ell$-restricted $A_{1} T$-system (iv) satisfies the following "twisted half-periodicity" relation:

$$
T_{j, k+\frac{N}{2}}=T_{\ell+1-j, k}
$$

where $N=2(\ell+3)$.

Proof. Let $\mathbf{k}=2 m+\mathbf{k}_{0}$ denote the unique even translate of $\mathbf{k}_{0}$ (with $\left.k_{j}=k_{j}^{(0)}+2 m\right)$ which contains the point $(\ell+1-j, k)$. If $k$ is odd, then $k_{j}=k-(j \bmod 2)$, otherwise, $k_{j}=k+1-(j \bmod 2)$ for all $j \in \mathbb{Z}$. The point $(\ell+1-j, k)$ is a local maximum if $k$ is odd, minimum otherwise. Let us compute $T_{j, k+\frac{N}{2}}$ via the formula (4.13). The cone of projection of $\left(j, k+\frac{N}{2}\right)$ onto the initial data segment $\left[j_{0}, j_{1}\right]$ is reflected once against each of the two lines $j=-1$ and $j=\ell+2$, and the edges of the cone intersect in the point $(\ell-j, k)$ as shown in Fig. 4 for odd $k$. For odd $k$, we find that:

$$
T_{j, k+\frac{N}{2}}=(P(-P))_{1,1}\left(-t_{\ell+1-j}\right)=T_{\ell+1-j, k}
$$

If $k$ is even, the reflected cone edges meet the path $\mathbf{k}$ respectively at points $(\ell-j, k+1)$ and $(\ell+2-j, k+1)$, thus leading to:

$$
T_{j, k+\frac{N}{2}}=\left(P V\left(t_{\ell-j}, t_{\ell+1-j}\right) U\left(t_{\ell+1-j}, t_{\ell+2-j}\right)(-P)\right)_{1,1}\left(-t_{\ell+2-j}\right)=t_{\ell+1-j}=T_{\ell+1-j, k}
$$

as well. The theorem follows.

We conclude that $T_{j, k+N}=T_{j, k}$ and the Theorem 4.2 follows. 


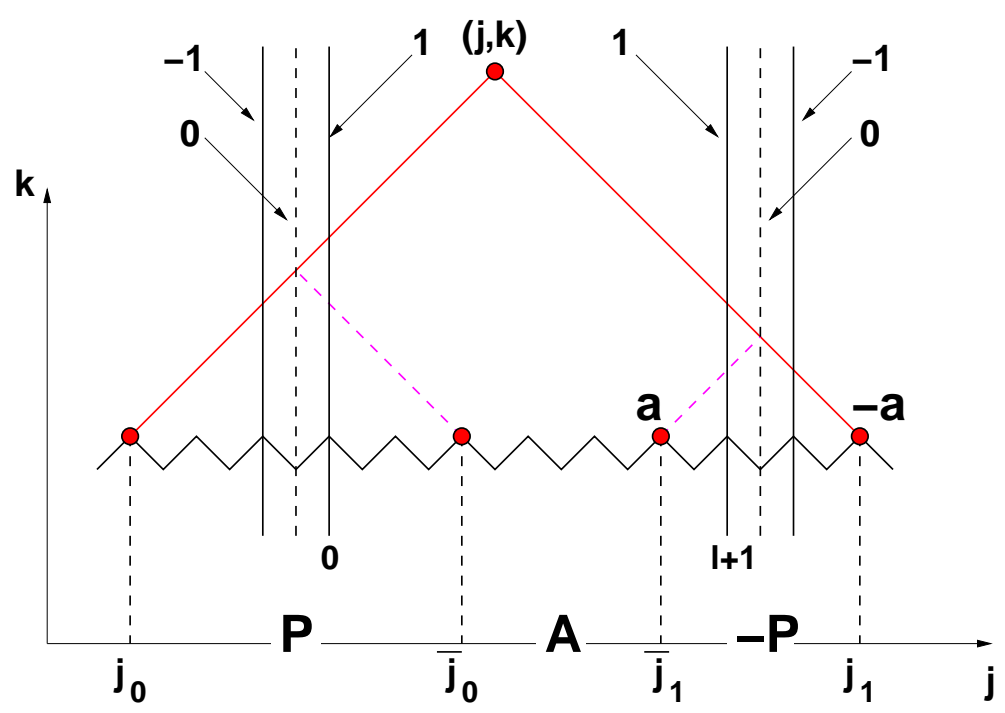

Figure 5: The cone of the projection of $(j, k)$ is reflected by both lines $j=-1$ and $j=\ell+2$ picking respectively a factor $P$ or $-P$ per reflection. After these two reflections, the edges of the cone meet the initial data path at $\left(\bar{j}_{0}, 1\right)$ and $\left(\bar{j}_{1}, 1\right)$ respectively. We have indicated the three contributions $P, A,-P$ from the formula (4.13), and the reflected boundary value $t_{\bar{j}_{1}}=a$.

\subsection{Positivity for $\mathrm{k}_{0}$ : proof of Theorem 4.3}

The first part of Theorem 4.3 follows by imposing simultaneously the symmetries of the initial values $t_{j}$ from both Theorems 4.5 and 4.7. As these guarantee the $\ell$-restricted boundary conditions, the result follows from uniqueness of the solution of the $\ell$-restricted system.

Let us now show that the solution $T_{j, k}$ of the $\ell$-restricted $A_{1} T$-system (iv) is a positive Laurent polynomial of the initial data along the path $\mathbf{k}_{0}$. Thanks to the half-periodicity property of Theorem 4.8, we may restrict ourselves to values of $k$ such that $0 \leqslant k \leqslant \frac{N}{2}$. In that case, the cone of projection of $(j, k)$ is reflected at most once against each line $j=-1$ and $j=\ell+2$. If no reflection occurs, the positivity is clear, as the solution is identical to that of the unrestricted $A_{1} T$-system. If only one reflection occurs, we are in the half-plane situation of Theorems 4.5 or 4.7, and positivity follows. We are left with the case of two reflections, as illustrated in Fig. 5 (case $\ell$ odd). As usual we denote by $j_{0}, j_{1}$ the minimum and maximum of the projection of $(j, k)$ onto the initial data path, and by $\bar{j}_{0}=-j_{0}-2$ and $\bar{j}_{1}=2(\ell+2)-j_{1}$ the reflected minimum and maximum of the projection, such that $0 \leqslant \bar{j}_{0} \leqslant \bar{j}_{1} \leqslant \ell+1$. Applying (4.13) and eliminating the left and right products involving $P$ and $-P$ leads to:

$$
T_{j, k}=(P A(-P))_{1,1}(-a)
$$

where

$$
A=V\left(t_{\bar{j}_{0}}, t_{\bar{j}_{0}+1}\right) U\left(t_{\bar{j}_{0}+1}, t_{\bar{j}_{0}+2}\right) \cdots U\left(t_{\bar{j}_{1}-1}, t_{\bar{j}_{1}}\right) \quad \text { and } \quad a=t_{\bar{j}_{1}}=-t_{j_{1}} .
$$






Figure 6: The cone of projection of $(j, k)$ is reflected by the line $j=-1$. As a result, only the values between $\bar{j}_{0}=-j_{0}-2$ and $j_{1}$ matter. We have also indicated the particular case of the point $(j=0, k)$ (right projection line is blue): the portion of boundary path for $j \geqslant \bar{j}_{0}$ within the projection is made of $m$ down steps followed by one up step.

As usual, the two signs cancel and leave us with a manifestly positive answer, and the second part of Theorem 4.3 follows.

\subsection{Positivity for k: proof of Theorem 4.4}

In the case of an arbitrary path $\mathbf{k}$ with associated initial conditions (4.5), we may repeat the same arguments as in the case $\mathbf{k}_{0}$. We first need to generalize the first part of Theorem 4.3 to the case of an arbitrary path $\mathbf{k}$. To this effect, Theorems 4.5 and 4.7 have the following counterparts for arbitrary $\mathbf{k}$ :

Theorem 4.9. The solution of the unrestricted $A_{1} T$-system (i) with boundary conditions $X_{\mathbf{k}^{+}}\left(\mathbf{t}^{+}\right)$(4.6) (resp. $\left.X_{\mathbf{k}^{-}}\left(\mathbf{t}^{-}\right)(4.7)\right)$ restricts to that of the right (resp. left) half-plane $A_{1}$ T-system (ii) (resp. (iii)) with boundary conditions $X_{\mathbf{m}}^{+}(\mathbf{u})$ (resp. $X_{\mathbf{m}}^{-}(\mathbf{u})$ ), where $\mathbf{u}, \mathbf{m}$ are the restrictions of $\mathbf{t}^{+}, \mathbf{k}^{+}$(resp. $\mathbf{t}^{-}, \mathbf{k}^{-}$) to the range $j \geqslant 1$ (resp. $j \leqslant \ell$ ). As such, the half-plane solutions are positive Laurent polynomials of their initial values.

Proof. The theorem is proved by showing that the reflection conditions on $\mathbf{k}$ and $t_{j}$ imply that $T_{0, k}=1$ (resp. $T_{\ell+1, k}=1$ ). As before, this is proved by use of the formula (4.13), upon noting that $\lim _{\epsilon \rightarrow 0} V(-1, \epsilon) U(\epsilon, 1)=P$ and $\lim _{\epsilon \rightarrow 0} V(1, \epsilon) U(\epsilon,-1)=-P$. We note also that, with the definition (4.12) and the symmetry properties of $\mathbf{k}$ and $t_{j}$, we have the following collapse relations:

$$
\begin{aligned}
M_{-j-3,-j-2}\left(t_{-j-3}, t_{-j-2}\right) & P M_{j, j+1}\left(t_{j}, t_{j+1}\right) \\
= & M_{-j-3,-j-2}\left(-t_{j+1},-t_{j}\right) P M_{j, j+1}\left(t_{j}, t_{j+1}\right)=P \\
M_{j, j+1}\left(t_{j}, t_{j+1}\right) & (-P) M_{2(\ell+2)-j, 2(\ell+2)-j+1}\left(t_{2(\ell+2)-j}, t_{2(\ell+2)-j+1}\right) \\
& =M_{j, j+1}\left(t_{j}, t_{j+1}\right)(-P) M_{2(\ell+2)-j, 2(\ell+2)-j+1}\left(-t_{j+1},-t_{j}\right)=-P
\end{aligned}
$$


For the right half-plane case, we have for $k \geqslant k_{0}, k$ odd:

$$
T_{0, k}=\left(\prod_{j=j_{0}}^{-3} M_{j, j+1}\left(t_{j}, t_{j+1}\right) P \prod_{j=0}^{j_{1}-1} M_{j, j+1}\left(t_{j}, t_{j+1}\right)\right)_{1,1} t_{j_{1}}=\left(P \prod_{j=\bar{j}_{0}}^{j_{1}-1} M_{j, j+1}\left(t_{j}, t_{j+1}\right)\right)_{1,1} t_{k},
$$

where $j_{0}, j_{1}$ denote the minimum and maximum of the projection of $(0, k)$ onto $\mathbf{k}$. Note that for $j$ between $\bar{j}_{0}=-j_{0}-2$ and $j_{1}$ the path $\mathbf{k}$ must be made of a number $m=j_{1}+j_{0}+1$ of down steps, followed by one up step (see Fig. 6 for an example). This leads to:

$$
T_{0, k}=\left[\left(\prod_{j=\bar{j}_{0}}^{j_{1}-2} V\left(t_{j}, t_{j+1}\right)\right) U\left(t_{j_{1}-1}, t_{j_{1}}\right)\right]_{2,1} t_{j_{1}}=\frac{1}{t_{j_{1}}} \times t_{j_{1}}=1
$$

The argument is similar for the left half-plane solution.

To prove positivity, let us consider a point $(j, k)$ above the path $\mathbf{k}$, namely with $k \geqslant k_{j}$. Then if $j_{0} \geqslant 0$ (resp. $j_{1} \leqslant \ell+1$ ), the solution $T_{j, k}$ is identical to that of the full plane, and positivity is granted. Otherwise, note that the remark 4.6 extends to the present cases: the collapse relations above have the effect of reflecting the cone of projection against the line $j=-1$ (resp. $j=\ell+2$ ), as indicated in Fig. 6. This gives the following expressions for $\bar{j}_{0}=-j_{0}-2$ and $\bar{j}_{1}=2(\ell+2)-j_{1}$ :

$$
\begin{array}{ll}
\text { right half - plane }: & T_{j, k}=\left(P N\left(\bar{j}_{0}, j_{1}\right)\right)_{1,1} t_{j_{1}} \\
\text { left half - plane : } & T_{j, k}=\left(N\left(j_{0}, \bar{j}_{1}\right)(-P)\right)_{1,1} t_{j_{1}}=\left(N\left(j_{0}, \bar{j}_{1}\right) P\right)_{1,1} t_{\bar{j}_{1}}
\end{array}
$$

which are both manifestly positive Laurent polynomials of the initial data.

To prove Theorem 4.4, we now superimpose the symmetry conditions for the two halfplane cases as described in Theorem 4.9. Let us show that the solution $T_{j, k}$ with arbitrary path initial data for $j \in[0, \ell+1]$ has the positive Laurent property.

The half-periodicity holds in general, so we may restrict ourselves to the case of a general path $\mathbf{k}$ and a point $(j, k)$ above it such that $0 \leqslant k-k_{j} \leqslant \frac{N}{2}$. In this case there is at most one reflection of the cone of projection of $(j, k)$ against each of the lines $j=-1$ and $j=\ell+2$. For no reflection at all, the solution is the same as that of the unrestricted $A_{1} T$-system case, which is manifestly positive. For one reflection against one of the lines, the solution is the same solution as that of the half-plane $A_{1} T$-system case, where positivity was established above. Finally for two reflections, we have:

$$
T_{j, k}=\left(P N\left(\bar{j}_{0}, \bar{j}_{1}\right)(-P)\right)_{1,1} t_{j_{1}}=\left(N\left(\bar{j}_{0}, \bar{j}_{1}\right)\right)_{2,2} t_{\bar{j}_{1}}
$$

which is manifestly positive. This completes the proof of Theorem 4.4. 


\section{$5 \quad \ell$-restricted T-system: the $A_{r}$ case}

Throughout this section, we study solutions of the $A_{r} T$-system $(1.1,1.6)$ with several types of boundary conditions, and with initial conditions of the form $X(\mathbf{t}):=X_{\mathbf{k}_{0}}(\mathbf{t})$ (1.7) or appropriate subsets thereof.

Let $S$ be a subset of $\mathbb{Z}$. We have the $T$-system

$$
T_{i, j, k+1} T_{i, j, k-1}=T_{i, j+1, k} T_{i, j-1, k}+T_{i+1, j, k} T_{i-1, j, k} \quad(i \in[1, r], k \in \mathbb{Z}, j \in S)
$$

with boundary conditions

$$
T_{0, j, k}=T_{r+1, j, k}=1 \quad(j \in S ; k \in \mathbb{Z})
$$

with possibly additional boundary conditions depending on $S$, and an initial condition $X^{S}(\mathbf{t})$, which is an assignment of formal variables $\mathbf{t}=\left(t_{i, j}\right)_{i \in[1, r], j \in S}$ to the points on the surface

$$
\mathbf{k}_{0}(S)=\left\{\left(i, j, k^{(0)}(i, j)\right): i \in[1, r], j \in S\right\} .
$$

We consider the following four cases:

(i) Unrestricted $A_{r} T$-system: $S=\mathbb{Z}$, there are no additional boundary conditions, and the initial condition $X(\mathbf{t})$ is an assignment of values to the variables on points of $\mathbf{k}_{0}$ :

$$
X(\mathbf{t}):\left\{T_{i, j, k_{i, j}^{(0)}}=t_{i, j} \quad(i \in[1, r] ; j \in \mathbb{Z})\right\} .
$$

(ii) Right half-space $A_{r} T$-system: $S=\mathbb{N}$, the additional boundary conditions are

$$
T_{i, 0, k}=1 \quad(i \in[1, r] ; k \in \mathbb{Z})
$$

and initial condition $X^{+}(\mathbf{t})$ is the assignment

$$
X^{+}(\mathbf{t}):\left\{T_{i, j, k_{i, j}^{(0)}}=t_{i, j} \quad(i \in[1, r] ; j \in S)\right\} .
$$

(iii) Left half-space $A_{r} T$-system: $S=(-\infty, \ell]$, the additional boundary conditions are

$$
T_{i, \ell+1, k}=1 \quad(i \in[1, r] ; k \in \mathbb{Z})
$$

and the initial conditions $X^{-}(\mathbf{t})$ are

$$
X^{-}(\mathbf{t}):\left\{T_{i, j, k_{i, j}^{(0)}}=t_{i, j} \quad(i \in[1, r] ; j \in S)\right\} .
$$

(iv) $\ell$-restricted $A_{r} T$-system: $S=[1, \ell]$, the additional boundary conditions are

$$
T_{i, 0, k}=T_{i, \ell+1, k}=1 \quad(i \in[1, r] ; k \in \mathbb{Z})
$$

and the initial conditions are $X^{[1, \ell]}(\mathbf{t})$

$$
X^{[1, \ell]}(\mathbf{t}):\left\{T_{i, j, k_{i, j}^{(0)}}=t_{i, j} \quad(i \in[1, r] ; j \in S)\right\} .
$$


Remark 5.1. In all the above cases, due to the form of the T-system as a three-term recursion, the solution of the system is uniquely determined by its initial conditions.

We will also consider the unrestricted $A_{r} T$-system (case (i)) with initial conditions $X(\mathbf{t})(5.2)$, where we impose certain relations on the variables $\mathbf{t}=\left(t_{i, j}\right)_{i \in[1, r], j \in \mathbb{Z}}$ :

- $\mathbf{t}^{+}$is $\mathbf{t}$ modulo the relations

$$
\begin{aligned}
& t_{r+1-i,-r-1-j}=(-1)^{r i} t_{i, j}, \quad(i \in[1, r] ; j \geqslant 0), \\
& t_{i, 0}=1, t_{i,-j}=0, \quad(i, j \in[1, r]) .
\end{aligned}
$$

- $\mathbf{t}^{-}$is $\mathbf{t}$ modulo the relations

$$
\begin{aligned}
& t_{r+1-i,-r-\ell-2+j}=(-1)^{r i} t_{i, \ell+1-j}, \quad(i \in[1, r] ; j \geqslant 0) \\
& t_{i, \ell+1}=1, t_{i, \ell+1+j}, \quad(i, j \in[1, r]) .
\end{aligned}
$$

- $\mathbf{t}^{[1, \ell]}$ is $\mathbf{t}$ modulo the relations

$$
\begin{aligned}
& t_{r+1-i,-r-1-j}=(-1)^{r i} t_{i, j}, \quad(i \in[1, r] ; j \geqslant 0), \\
& t_{i, 2(r+\ell+2)+j}=t_{i, j}, \quad(i \in[1, r] ; j \in \mathbb{Z}), \\
& t_{i, 0}=t_{i, \ell+1}=1, \quad(i \in[1, r]), \\
& t_{i,-j}=0, \quad(i, j \in[1, r]) .
\end{aligned}
$$

Remark 5.2. The relations satisfied by $\mathbf{t}^{[1, \ell]}$ correspond to simultaneously imposing the relations of $\mathbf{t}^{+}$and $\mathbf{t}^{-}$.

Example 5.3. Initial data of type $\mathbf{t}^{+}$for the case $r=3$ has the form (with the $i$ direction is from bottom to top, and $j$ direction is from left to right):

$$
\begin{array}{lrrrrrrrrrrrrrrrr}
\cdots & -t_{1,5} & -t_{1,4} & -t_{1,3} & -t_{1,2} & -t_{1,1} & -1 & 0 & 0 & 0 & 1 & t_{3,1} & t_{3,2} & t_{3,3} & t_{3,4} & t_{3,5} & \cdots \\
\cdots & t_{2,5} & t_{2,4} & t_{2,3} & t_{2,2} & t_{2,1} & 1 & 0 & 0 & 0 & 1 & t_{2,1} & t_{2,2} & t_{2,3} & t_{2,4} & t_{2,5} & \cdots \\
\cdots & -t_{3,5} & -t_{3,4} & -t_{3,3} & -t_{3,2} & -t_{3,1} & -1 & 0 & 0 & 0 & 1 & t_{1,1} & t_{1,2} & t_{1,3} & t_{1,4} & t_{1,5} & \cdots
\end{array}
$$

Example 5.4. Initial data of the type $\mathbf{t}^{[1, \ell]}$ for the case $r=3, \ell=3$ has the form

$$
\begin{aligned}
& \begin{array}{lllll|lllll|llllllllll}
-t_{1,1} & -1 & 0 & 0 & 0 & 1 & t_{3,1} & t_{3,2} & t_{3,3} & 1 & 0 & 0 & 0 & -1 & -t_{1,3} & -t_{1,2} & -t_{1,1} & -1 & 0 & 0
\end{array} \\
& \begin{array}{lllllllllll|lllllllllll}
\cdots & t_{2,1} & 1 & 0 & 0 & 0 & 1 & t_{2,1} & t_{2,2} & t_{2,3} & 1 & 0 & 0 & 0 & 1 & t_{2,3} & t_{2,2} & t_{2,1} & 1 & 0 & 0 & \cdots
\end{array} \\
& \begin{array}{llllllllll|llllllllll}
-t_{3,1} & -1 & 0 & 0 & 0 & 1 & t_{1,1} & t_{1,2} & t_{1,3} & 1 & 0 & 0 & 0 & -1 & -t_{3,3} & -t_{3,2} & -t_{3,1} & -1 & 0 & 0
\end{array}
\end{aligned}
$$

This array has period $2(\ell+r+2)=16$ along the horizontal $(j$-)direction. The vertical bars indicate the domain corresponding to the $\ell$-restricted $A_{r}$ T-system (iv) initial data.

As in the $A_{1}$ case, the aim of this section is to use the known network solution for the unrestricted system (i) to obtain that for the other boundary conditions (ii,iii,iv). 


\subsection{Equivalent initial data and main theorems}

Here, we give the line of argument used to prove the periodicity theorems 1.4 and 1.5.

Lemma 5.5. The solutions of the T-system (i) with initial conditions $X\left(\mathbf{t}^{+}\right)$satisfy

$$
T_{1,0, k}=1 \quad(k \in 2 \mathbb{Z}+1), \quad T_{1,-j, k}=0, \quad(j \in[1, r] ; k \in 2 \mathbb{Z}+j+1) .
$$

The determinant formula (1.8) and the Lemma imply that $T_{i, 0, k}=1$ for all $i \in[1, r]$ and $k \in 2 \mathbb{Z}+i$. The proof of this Lemma is given in Section 5.5.

Theorem 5.6. The solutions $T_{i, j, k}$ of the unrestricted $A_{r}$ T-system (i) as a function of the initial conditions $X\left(\mathbf{t}^{+}\right)$are equal, when $j>0$, to the solutions $T_{i, j, k}$ of the right half-space $A_{r}$ T-system (ii) with initial conditions $X^{+}(\mathbf{t})$.

Proof. Given Lemma 5.5, the theorem follows from the uniqueness of the solutions (Remark 5.1) of the half-plane $T$-system with initial data $X^{+}(\mathbf{t})$.

Theorem 5.7. The solutions $T_{i, j, k}$ of the unrestricted $A_{r}$ T-system (i) as a function of initial conditions $X\left(\mathbf{t}^{-}\right)$are equal, when $j \leqslant \ell$, to the solutions $T_{i, j, k}$ of the left half-space $A_{r} T$-system (iii) with initial conditions $X^{-}(\mathbf{t})$.

Proof. Let $\sigma$ be the following endomorphism of $[1, r] \times \mathbb{Z} \times \mathbb{Z}$ :

$$
\sigma(i, j, k)= \begin{cases}(i, \ell+1-j, k), & \ell \text { odd } \\ (i, \ell+1-j, 1-k), & \ell \text { even }\end{cases}
$$

Then $\sigma\left(\mathbf{k}_{0}\right)=\mathbf{k}_{0}$ and $\sigma$ is also a symmetry of the unrestricted $A_{r} T$-system (i). It acts on $\mathbf{t}$ in the natural way, $\sigma\left(t_{i, j}\right)=t_{i, \ell+1-j}$, and takes initial data of the form $\mathbf{t}^{+}$to data of the form $\mathbf{t}^{-}$. The Theorem follows from application of $\sigma$ to the result of Theorem 5.7.

Using the map $\sigma$ together with Lemma 5.5 we see that for all $k$ of appropriate parity,

$$
T_{i, \ell+1, k}=1, \quad T_{i, \ell+1+j, k}=0 \quad(j \in[1, r]) .
$$

Lemma 5.5 and its reflected version (5.18) imply the following result for $\ell$-restricted $A_{r}$ $T$-system solutions:

Theorem 5.8. The solutions $T_{i, j, k}$ of the unrestricted $A_{r}$ T-system (i) as a function of initial conditions $X\left(\mathbf{t}^{[1, \ell]}\right)$ are equal, when $j \in[1, \ell]$, to the solutions of the $\ell$-restricted $A_{r}$ T-system (iv) with initial conditions $X^{[1, \ell]}(\mathbf{t})$.

Proof. This follows from Remark 5.2 and the uniqueness of the solutions.

We will also prove certain positivity results for the solutions of the $A_{r} T$-systems of types (ii)-(iv), using the explicit network solution of (i):

Theorem 5.9. The solutions $T_{i, j, k}$ of $A_{r} T$-system of type (ii) and (iii) with initial conditions $X^{+}(\mathbf{t})$ or resp. $X^{-}(\mathbf{t})$, are Laurent polynomials of the initial data $\mathbf{t}$, with nonnegative integer coefficients.

From the network solution with the two half-plane boundaries superimposed, this implies the positivity Theorem 1.5. The proof appears in Section 5.7. 


\subsection{A regularized network matrix}

Initial data of the form $\mathbf{t}^{+}$contains zeros. In order to define network matrices depending on this initial data, the matrices $U$ and $V$ cannot be used directly. To define the matrices $P_{j}:=N(-j, 0)$ (with $j \in[0, r+1]$ ) depending on $\mathbf{t}^{+}$, we use a limiting procedure as in the case of $A_{1}$. First, define regularized initial data by replacing the vanishing conditions of Theorem 5.6 by non-zero values forming an array $\left(a_{i, j}\right)$ compatible with the $T$-system. The regularized network matrices $P_{j}(\{a\})$ have a well-defined limit when $a_{i, j} \rightarrow 0$.

\subsubsection{Regularized initial data}

Definition 5.10. We consider the array $\left(a_{i,-j}\right)_{i, j \in[0, r+1]}$ such that:

$$
\begin{aligned}
& a_{i, 0}=a_{0,-j}=a_{r+1,-j}=1, \quad i, j \in[0, r+1] \\
& a_{i-1,-j} a_{i+1,-j}+a_{i,-j-1} a_{i,-j+1}=0, \quad i, j \in[1, r] .
\end{aligned}
$$

The values of $a_{i, j}$ are determined recursively from the column with $j=-1$. Define $a_{i}=a_{i,-1}$ for $i \in[1, r]$. Then

$$
a_{i, j}=\epsilon_{i, j} \prod_{\ell=0}^{\operatorname{Min}(i,-j, r+1-i, r+1+j)-1} a_{|i+j|+1+2 \ell}
$$

where $\epsilon_{i, j} \in\{-1,1\}$ is the solution to the recursion relations $\epsilon_{i, j-1}=-\epsilon_{i-1, j} \epsilon_{i+1, j} / \epsilon_{i, j+1}$, while $\epsilon_{i, 0}=\epsilon_{i,-1}=\epsilon_{0, j}=\epsilon_{r+1, j}=1$ for all $i \in[0, r+1]$ and $j \in[-r-1,0]$. In particular, $a_{i,-r-1}=\epsilon_{i,-r-1}=(-1)^{r i}$.

Example 5.11. For the case $r=3$, we have the following array (represented with index $i$ from bottom to top and $j$ from left to right):

$$
\left(a_{i,-j}\right)_{0 \leqslant i, j \leqslant 4}=\left(\begin{array}{ccccc}
1 & 1 & 1 & 1 & 1 \\
-1 & a_{1} & -a_{2} & a_{3} & 1 \\
1 & -a_{2} & -a_{1} a_{3} & a_{2} & 1 \\
-1 & a_{3} & -a_{2} & a_{1} & 1 \\
1 & 1 & 1 & 1 & 1
\end{array}\right)
$$

and for the case $r=4$,

$$
\left(a_{i,-j}\right)_{0 \leqslant i, j \leqslant 5}=\left(\begin{array}{cccccc}
1 & 1 & 1 & 1 & 1 & 1 \\
1 & -a_{1} & a_{2} & -a_{3} & a_{4} & 1 \\
1 & -a_{2} & -a_{1} a_{3} & -a_{2} a_{4} & a_{3} & 1 \\
1 & -a_{3} & -a_{2} a_{4} & -a_{1} a_{3} & a_{2} & 1 \\
1 & -a_{4} & a_{3} & -a_{2} & a_{1} & 1 \\
1 & 1 & 1 & 1 & 1 & 1
\end{array}\right)
$$

Remark 5.12. As apparent from the formula (5.19), the expression for $a_{i, j}$ involves only $a_{k}$ 's with a fixed parity of $k$, namely $k=i+j+1 \bmod 2$. 
We define the regularlized initial data $\mathbf{t}^{+}(\mathbf{a})$ as follows. We keep the symmetry requirements (5.9) but replace the zeros in (5.10) with arrays satisfying Definition 5.10:

$$
t_{i, j}=a_{i, j} \quad(i \in[1, r] ; j \in[-r-1,0])
$$

\subsubsection{Regularized network matrices}

For each $j \in[0, r+1]$, define $P_{j}(\{a\})=N(-j, 0)(\{a\})$ corresponding to the network with initial values $\mathbf{t}^{+}(\mathbf{a})$ as follows. Let

$$
N_{i, j}(\{a\})=\left\{\begin{array}{ll}
U_{i}\left(a_{i, j-1}, a_{i, j}, a_{i+1, j-1}\right) & \text { if } i+j=1 \bmod 2 ; \\
V_{i}\left(a_{i-1, j}, a_{i, j-1}, a_{i, j}\right) & \text { otherwise, }
\end{array} \quad(i,-j \in[1, r]) .\right.
$$

The regularized network matrix is the product of matrices

$$
P_{j}(\{a\})=\prod_{k=-j+1}^{0} \prod_{i=1}^{r} N_{i, k}(\{a\}), \quad j \in[0, r+1]
$$

taken with the indicated order. With this definition, the matrix corresponding to the lower right corner of the network is $U_{1}\left(a_{1}, 1, a_{2}\right)$, as it corresponds to $i=1$ and $j=0$ in (5.23).

Lemma 5.13. Within the domain $j \in[-r-1,0]$ of the regularized network, each "diamond" of the form $U_{i}(a, b, v) V_{i}(u, b, c)$ or $V_{i}(u, a, b) U_{i}(b, c, v)$, with $a c+u v=0$, has elements in $\mathbb{Z}\left[b, u, v, c^{-1}\right]$. In particular, only $c$ may occur as a denominator.

Proof. We compute the $U V$ diamond matrix:

$$
U(a, b, v) V(u, b, c)=\mathbf{a}
$$

where the $(2,2)$ matrix element vanishes, due to $a c+u v=0$. Analogously,

$$
V(u, a, b) U(b, c, v)=\mathbf{a}\left\langle\mathbf{c}=\left(\begin{array}{ll}
0 & \frac{u}{c} \\
\frac{v}{c} & \frac{b}{c}
\end{array}\right)\right.
$$

where the $(1,1)$ matrix element vanishes, due to $a c+u v=0$.

Theorem 5.14. The entries of the matrices $P_{j}(\{a\}), j \in[0, r+1]$, are polynomials of the $a_{i}$ 's, $i \in[1, r]$. Therefore, the matrices

$$
P_{j}:=\lim _{a_{1}, a_{2}, \ldots, a_{r} \rightarrow 0} P_{j}(\{a\})
$$

are well defined. 
Proof. We concentrate on the portion $[-j, 0]$ of the regularized network. It may be decomposed into two types $(U V$ or $V U)$ of diamonds as follows:
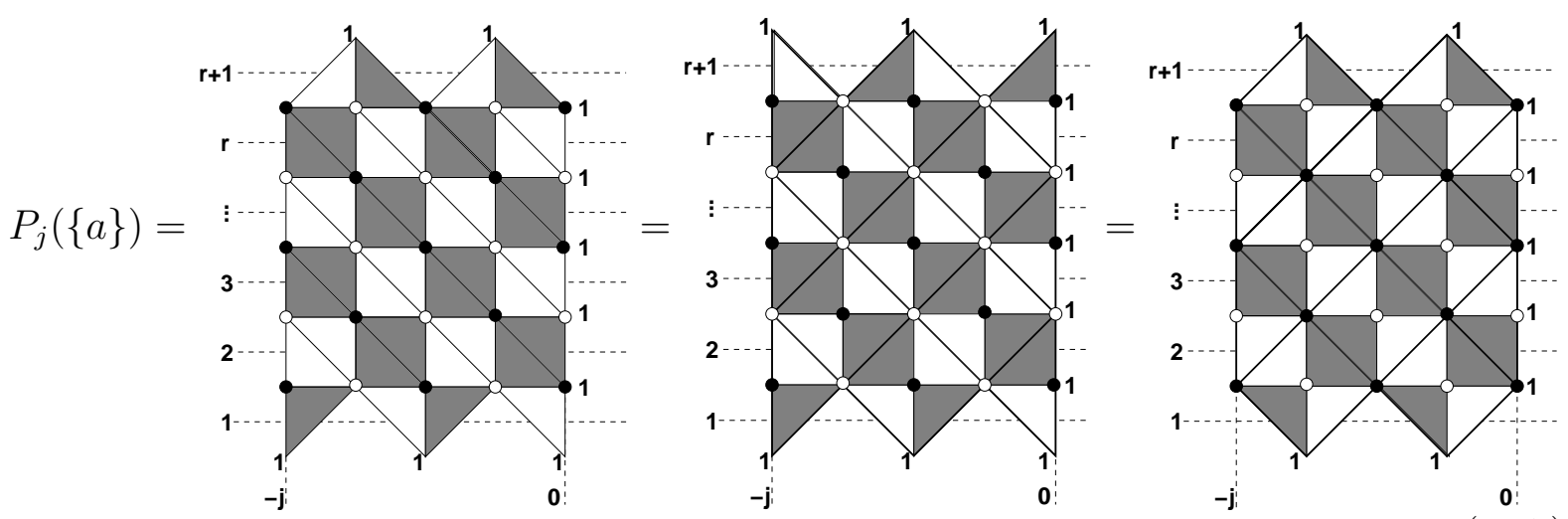

$(5.27)$

Due to Lemma 5.13, the first decomposition gives rise to matrix elements with denominators corresponding to values of $a_{i, j}$ at white circles (with $i+j=1 \bmod 2$ ), while in the second the only possible denominators correspond to values of $a_{i, j}$ at black circles (with $i+j=0 \bmod 2$ ). The unpaired column of $U$ and $V$ matrices on the right has only 1 as possible denominator, due to the boundary condition along the $j=0$ column.

From (5.19) and Remark 5.12, the matrix elements for the first expression for $P(\{a\})$ may only have denominators that are monomials of the $a_{2 i}$ 's, while the second expression may only have denominators that are monomials of the $a_{2 i+1}$ 's. We conclude that none of these denominators may occur in $P_{j}(\{a\})$, which is therefore a polynomial of the $a$ 's, and the theorem follows.

Let $P$ denote the $(r+1) \times(r+1)$ matrix with entries:

$$
[P]_{i, j}=(-1)^{(r-1)(i-1)} \delta_{i+j, r+2}
$$

Clearly, $P^{2}=\mathbb{I}$, and when $r=1$ the above definition agrees with (4.14).

\section{Lemma 5.15.}

$$
\operatorname{det}\left(P_{r+1}(\{a\})=\operatorname{det}(P)=(-1)^{\frac{r(r+1)(r+2)}{2}}\right.
$$

Proof. The determinants of the $U$ and $V$ matrices are equal to the product of weights of the horizontal edges. Therefore $\operatorname{det}\left(P_{r+1}(\{a\})\right.$ is a product over the weights of all horizontal edges of the regularized network, each of which is equal to 1 or $a_{i, j-1} / a_{i, j}$ where $i \in[1, r]$ and $j \in[-r, 0]$. Therefore,

$$
\operatorname{det}\left(P_{r+1}(\{a\})=\prod_{i-1}^{r} \prod_{j=-r}^{0} \frac{a_{i, j-1}}{a_{i, j}}=\prod_{i=1}^{r} \frac{a_{i,-r-1}}{a_{i, 0}}=\prod_{i=1}^{r}(-1)^{r i},\right.
$$

and the lemma follows. 
We also note the following useful properties of $U, V$ matrices:

$$
\begin{aligned}
& U(a, b, c)=U(\lambda a, \lambda b, \lambda c) \quad V(a, b, c)=V(\lambda a, \lambda b, \lambda c) \\
& U(a, b, c)^{-1}=U(b, a,-c) \quad V(a, b, c)^{-1}=V(-a, c, b)
\end{aligned}
$$

We give below a pictorial proof of the following formula for $P_{r+1}(\{a\})$ :

Theorem 5.16. Given $r=2 s+\epsilon$ with $\epsilon \in\{0,1\}$, the matrix $P_{r+1}(\{a\})$ is

$$
P_{r+1}(\{a\})=\left(\prod_{i=1-\epsilon}^{s} U_{2 i}\left(1,1,(-1)^{\epsilon+1} a_{2(s-i)+1}\right)\right) P\left(\prod_{i=1}^{s} U_{2 i}\left(1,1, a_{2 i}\right)\right)
$$

Proof. In the case $r=2 s$, using the formula for the inverse of $U$ (5.30), the statement of the theorem will follow if we prove that

$$
\Pi_{r+1}(\{a\})=\left(\prod_{i=1}^{s} U_{2 i}\left(1,1, a_{2(s-i)+1}\right)\right) P_{r+1}(\{a\})\left(\prod_{i=1}^{s} U_{2 i}\left(1,1,-a_{2 i}\right)\right)
$$

is equal to $P$, independently of the $a$ 's. Analogously, when $r=2 s+1$, using also the projectivity property (5.29) with $\lambda=-1$, the theorem boils down to proving that

$$
\Pi_{r+1}(\{a\})=\left(\prod_{i=0}^{s} U_{2 i+1}\left(-1,-1, a_{2(s-i)+1}\right)\right) P_{r+1}(\{a\})\left(\prod_{i=1}^{s} U_{2 i}\left(1,1,-a_{2 i}\right)\right)
$$

is equal to $P$, independently of the $a$ 's. The matrix $\Pi_{r+1}(\{a\})$ corresponds in both cases to an augmented network matrix. We illustrate the network below for the cases $r=7,8$ :

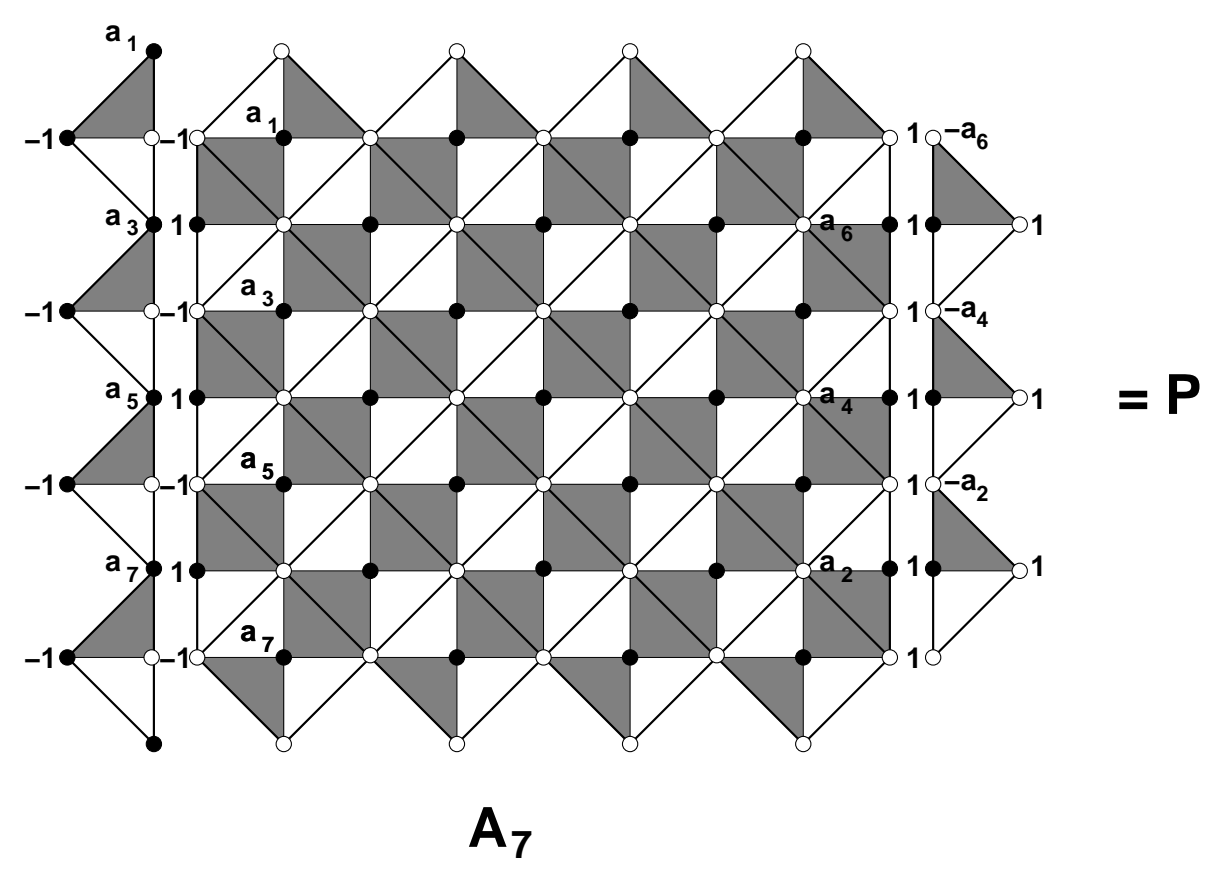




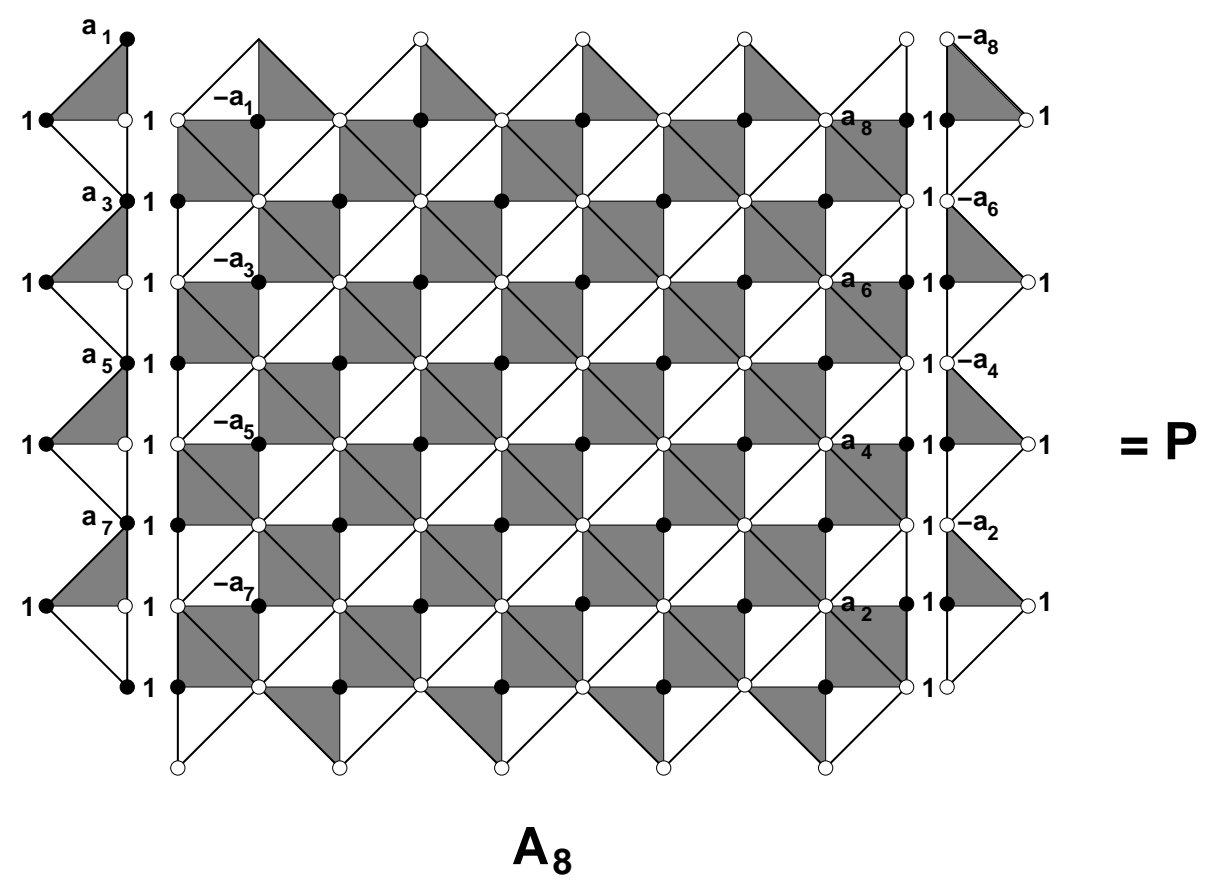

(Note that the actual values of the added pieces are compatible with both $U V$ and $V U$ diamond decompositions of Theorem 5.14.). The matrix elements of $\Pi_{r+1}(\{a\})$ are all polynomials of the $a$ 's. This is readily seen from Theorem 5.14 for $k=r+1$, together with the explicit form of $U(1,1, x)=U(-1,-1,-x)=\left(\begin{array}{ll}1 & 0 \\ x & 1\end{array}\right)$ which has only polynomial entries of $x$. This also implies that

$$
\operatorname{det}\left(\Pi_{r+1}(\{a\})\right)=\operatorname{det}\left(P_{r+1}(\{a\})=(-1)^{\frac{r(r+1)(r+2)}{2}}\right.
$$

by Lemma 5.15 .

To compute $\Pi_{r+1}(\{a\})$, we use the pictorial representation II (2.10) for the the nonzero matrix elements of the $U, V$ matrices, and we note that the network chips for the $U V$ and $V U$ diamonds (5.25) and (5.26) may be represented as:

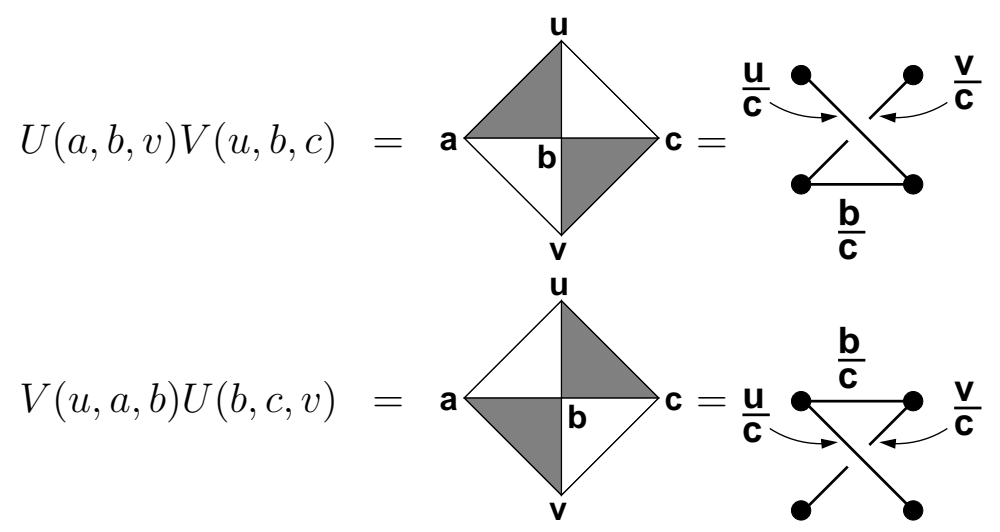

where the missing horizontal edge on the regularized network is due to vanishing condition $u v+a c=0$. The two different $(U V$ or $V U)$ diamond decompositions of $\Pi_{r+1}(\{a\})$ in 
pictorial representation II, in the case $r=8$ are:
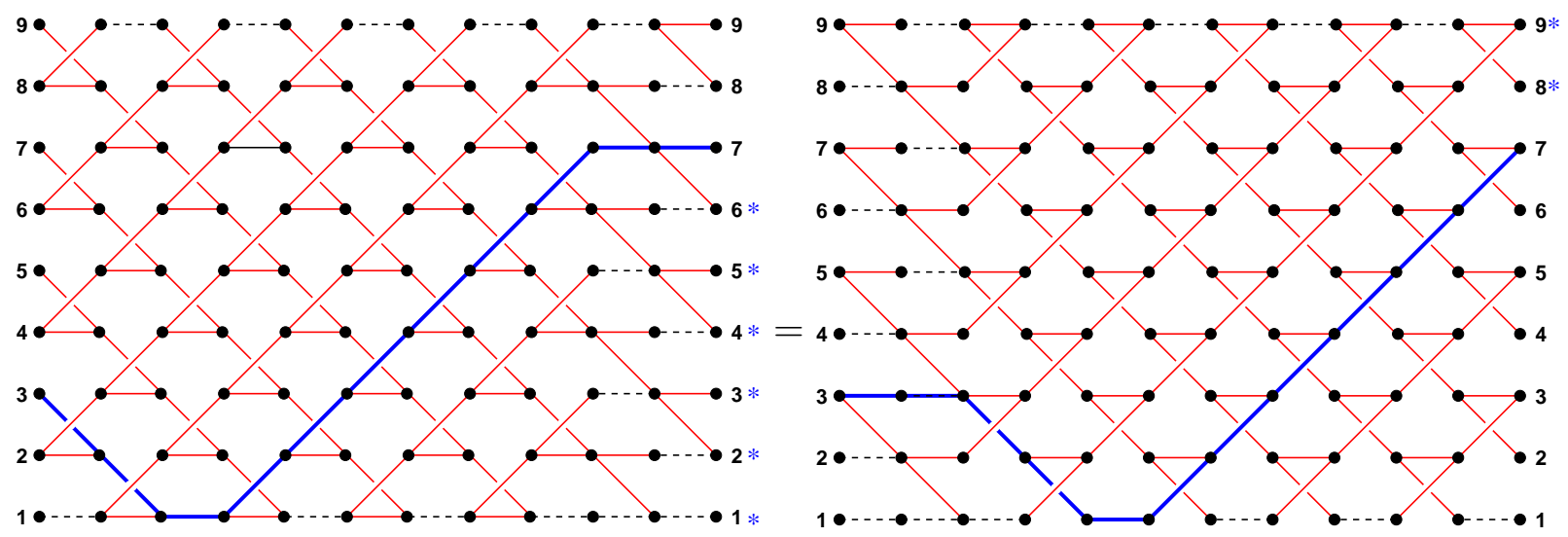

where each edge is weighted with a Laurent monomial of the $a$ 's. The left diagram shows that there are paths from vertex $i$ on the left to vertex $i^{\prime}$ on the right only if $i^{\prime} \leqslant r+2-i$, whereas the right diagram shows that there are paths from $i$ to $i^{\prime}$ only if $i^{\prime} \geqslant r+2-i$, for each $i$. Therefore there are non-zero weighted paths only from each vertex $i$ to $r+2-i$. The corresponding path is unique. Equivalently, $\Pi_{r+1}(\{a\})_{i, j}=0$ unless $j=r+2-i$. This is illustrated in the above picture by highlighting in thick solid blue line the unique contributing path $3 \rightarrow 7$, while the other attainable points via paths starting at vertex 3 are indicated by blue asterisks.

Moreover, the total weight of the single contributing path $i \rightarrow r+2-i$, equal to the matrix element $\left[\Pi_{r+1}(\{a\})\right]_{i, r+2-i}$, is a monomial of the $a$ 's (with only non-negative powers, as the entries of $\Pi_{r+1}(\{a\})$ are all polynomials). The determinant of $\Pi_{r+1}(\{a\})$ reads:

$$
\operatorname{det}\left(\Pi_{r+1}(\{a\})\right)=(-1)^{r(r+1) / 2} \prod_{i=1}^{r+1} \Pi_{r+1}(\{a\})_{i, r+2-i}
$$

Comparing this with (5.31), we see that none of the matrix elements $\Pi_{r+1}(\{a\})_{i, r+2-i}$ vanish, and each of them has value \pm 1 . To conclude, we note that the face weights cancel out along the paths as the product over step weights is telescopic, leaving us with only the ratio: (leftmost face variable)/(rightmost face variable). Inspecting the signs from the boundary faces carefully, we finally conclude that $\Pi_{r+1}(\{a\})=P$.

Corollary 5.17. We have

$$
\lim _{a_{1}, a_{2}, \ldots, a_{r} \rightarrow 0} P_{r+1}(\{a\})=P
$$

Proof. We use the expressions of Theorem 5.16, and note that $U_{i}(1,1,0)=U(-1,-1,0)=$ $\mathbb{I}$ for all $i \in[1, r]$.

Lemma 5.18. For each $j \in[1, r]$,

$$
\left(P_{j}\right)_{1, i}=\lim _{a_{1}, a_{2}, \ldots, a_{r} \rightarrow 0}\left(P_{j}(\{a\})\right)_{1, i}=\delta_{i, 2\left\lfloor\frac{j}{2}\right\rfloor+1} .
$$


Proof. We give a pictorial proof. Use the diamond decomposition of the networks (5.27) in pictorial representation II with chips (5.33). For even and odd $j$ 's, we get respectively (here $j=4,5$ ):
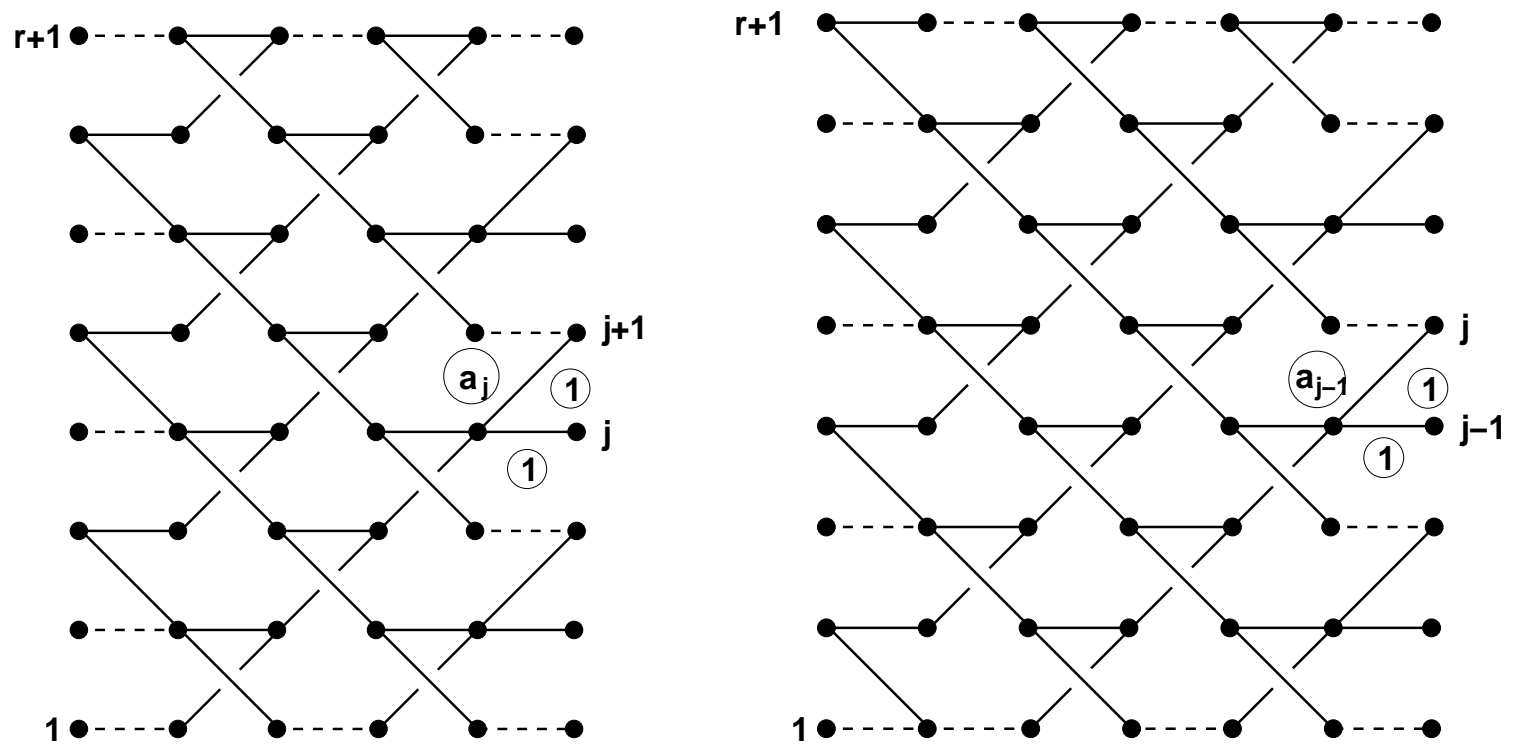

There are exactly two paths from $1 \rightarrow k$, with $k=j, j+1$ if $j$ is even, and $k=j-1, j$ is $j$ is odd. The face labels of the last chip are represented inside circles. The weights of the two paths in the case of even (resp. odd) $j$ are identical except for their last step, weighted respectively by: 1 if the last step is diagonal and $a_{j}$ (resp. $a_{j-1}$ ) if it is horizontal. Therefore only the path ending with a diagonal step contributes in the limit $a_{i} \rightarrow 0$. Moreover, the weights along this remaining path, due to (5.33), are of the form $v / c$ and therefore their product is telescopic and leaves us with (leftmost face label)/ $($ rightmost face label $)=1$. This proves the lemma.

\subsection{The reflected network matrix}

We can give a similar definition of the regularized network matrix for $N(\ell+1, \ell+r+2)$ of the form $N(\ell+1, \ell+r+2)(\{b\})$ for a compatible array $\left(b_{i, j}\right)$. In order to satisfy the boundary conditions in the initial data $\mathbf{t}^{-}$, choose $N(\ell+1, \ell+r+2)(\{b\})$ to be the regularized network matrix with $b_{i, j}$ an array defined as follows:

$$
b_{i, j}=(-1)^{r i} a_{i, j-(\ell+r+2)}, \quad i \in[0, r+1], j \in[\ell+1, \ell+r+2] .
$$

where $a_{i, j}$ is given by Definition 5.10. This ensures that $b_{i, \ell+1}=1$ and $b_{i, \ell+r+2}=(-1)^{i}$ when $r$ is odd.

Let $S$ be the matrix with entries:

$$
[S]_{i, j}=(-1)^{i-1} \delta_{i, j} \quad(i, j \in[1, r+1]) .
$$

Clearly,

$$
S^{2}=\mathbb{I} \quad S P=(-1)^{r} P S .
$$


Moreover,

$$
\begin{aligned}
S U_{i}(a, b, c) S & =U_{i}(a, b,-c)=U_{i}(-a,-b, c) \\
S V_{i}(a, b, c) S & =V_{i}(a,-b,-c)=V_{i}(-a, b, c) .
\end{aligned}
$$

Lemma 5.19. The regularized network matrix $\tilde{P}_{r+1}(\{b\})=N(\ell+1, \ell+r+2)(\{b\})$ defined above satisfies:

$$
\lim _{b_{1}, \ldots, b_{r} \rightarrow 0} \tilde{P}_{r+1}(\{b\})=(-1)^{r} P .
$$

Proof. The lemma is clear for even $r$, as $N(\ell+1, \ell+r+2)(\{b\})=P_{r+1}(\{a\})$ with $b_{i, j}=a_{i, j-(\ell+r+2)}$. For odd $r$, we have:

$$
S P_{r+1}(\{a\}) S=N(\ell+1, \ell+r+2)(\{b\}) \quad \text { where } \quad b_{i, j}=(-1)^{i} a_{i, j-(\ell+r+2)},
$$

with $S$ as in (5.28). Indeed, eqns. (5.36) and (5.37) allow us to interpret the conjugate action of $S$ as flipping the sign of all array entries along every other row, say $i=1,3, \ldots, r$. Taking the $a \rightarrow 0$ limit in both cases leads respectively to $P_{r+1}(\{0\})=P$ for even $r$ and $S P S=-P$ for odd $r$ by (5.35), and the lemma follows.

It will also be useful to have the corresponding version of Lemma 5.18. Define the family of regularized network matrices $\tilde{P}_{j}(\{b\})=N(\ell+1, \ell+1+j)(\{b\}), j=0,1,2, \ldots, r$, with $\tilde{P}_{0}(\{b\})=\mathbb{I}$. Each $b_{i, j}$ is a signed monomial of the variables $\left\{b_{k}:=b_{k, \ell+2}\right\}$. In particular, $b_{1, \ell+1+j}=(-1)^{j-1} b_{j}$.

Lemma 5.20. The limit $b_{j} \rightarrow 0$ of the regularized network matrices is

$$
\lim _{b_{1}, \ldots, b_{r} \rightarrow 0}\left[\tilde{P}_{j}(\{b\})\right]_{i, 1} b_{1, \ell+1+j}=\delta_{i, a_{\ell}(j)} \quad j \in[1, r] .
$$

where

$$
a_{\ell}(x)=\left\{\begin{array}{cc}
2\left\lfloor\frac{x+1}{2}\right\rfloor & \text { if } \ell \text { is even } \\
2\left\lfloor\frac{x}{2}\right\rfloor+1 & \text { if } \ell \text { is odd }
\end{array}\right.
$$

Proof. The proof is very similar to that of Lemma 5.18 The difference is that one must distinguish between odd $\ell$ (the actual reflection of the case of Lemma 5.18) and even $\ell$, in which $U$ and $V$ matrices are interchanged. The telescopic products of weights for the remaining path ending at 1 is $1 / b_{1, \ell+1+j}$, where and the denominator is cancelled by the prefactor above.

\subsection{Collapse relations}

The following relations may be verified by direct calculation.

$$
\begin{aligned}
& P U_{i}(a, b, c) P=V_{r+1-i}\left((-1)^{r-1} c, a, b\right), \\
& P V_{i}(a, b, c) P=U_{r+1-i}\left(b, c,(-1)^{r-1} a\right) .
\end{aligned}
$$


Lemma 5.21. Let $i \in[1, r]$ and $j \geqslant 1$. Given initial data of the form $\mathbf{t}^{+}$,

$$
\begin{aligned}
V_{r+1-i}\left(t_{r-i,-r-j}, t_{r+1-i,-r-1-j}, t_{r+1-i,-r-j}\right) P U_{i}\left(t_{i, j-1}, t_{i, j}, t_{i+1, j-1}\right) & =P, \\
U_{r+1-i}\left(t_{r+1-i,-r-1-j}, t_{r+1-i,-r-j}, t_{r+2-i,-r-1-j}\right) P V_{i}\left(t_{i-1, j}, t_{i, j-1}, t_{i, j}\right) & =P .
\end{aligned}
$$

Proof. Multiplying the relation (5.41) from the left by the inverse of $V_{r+1-i}\left((-1)^{r-1} c, b, a\right)$ and similarly $(5.42)$ by the inverse of $U_{r+1-i}\left(b, c,(-1)^{r-1} a\right)$ using (5.30) gives:

$$
V_{r+1-i}\left((-1)^{r} c, b, a\right) P U_{i}(a, b, c)=P, \quad U_{r+1-i}\left(c, b,(-1)^{r} a\right) P V_{i}(a, b, c)=P .
$$

The reflection symmetry on $\mathbf{t}^{+}(5.9)$ means that

$$
\begin{gathered}
t_{r-i,-r-j}=(-1)^{r(i+1)} t_{i+1, j-1}, \quad t_{r+1-i,-r-1-j}=(-1)^{r i} t_{i, j}, \\
t_{r+1-i,-r-j}=(-1)^{r i} t_{i, j-1}, \quad t_{r+2-i,-r-1-j}=(-1)^{r(i-1)} t_{i-1, j} .
\end{gathered}
$$

The Lemma follows from the projective property (5.29) with $\lambda=(-1)^{r i}$.

\subsection{Proof of Lemma 5.5}

We prove the two statements in the Lemma.

Lemma 5.22. The solutions of the unrestricted $A_{r}$ T-system of type (i) with initial conditions $X\left(\mathbf{t}^{+}\right)$have the property that $T_{1,0, k}=1$ for all odd $k$.

Proof. By reflection symmetry, it is only necessary to consider $k>0$. The projection of the point $(1,0, k)$ onto $\mathbf{k}_{0}$ is the portion with $j \in[-k+1, k-1]$ :

$$
T_{1,0, k}=[N(-k+1, k-1)]_{1,1} t_{1, k-1} .
$$

There are two cases to consider.

case $1: k-1>r$. In this case,

$$
\begin{aligned}
N(-k+1, k-1) & =N(-k+1,-r-1) N(-r-1,0) N(0, k-r-2) N(k-r-2, k-1) \\
& =N(-k+1,-r-1) P N(0, k-r-2) N(k-r-2, k-1)
\end{aligned}
$$

using Corollary 5.17. Lemma 5.21 implies $N(-k+1,-r-1) P N(0, k-r-2)=P$ for the initial data $\mathbf{t}^{+}$. We deduce that $T_{1,0, k}=[N(k-r-2, k-1)]_{r+1,1} t_{1, k-1}$. Let us examine the network corresponding to $N(k-r-2, k-1)$. As before, let us decompose it into diamonds of the form

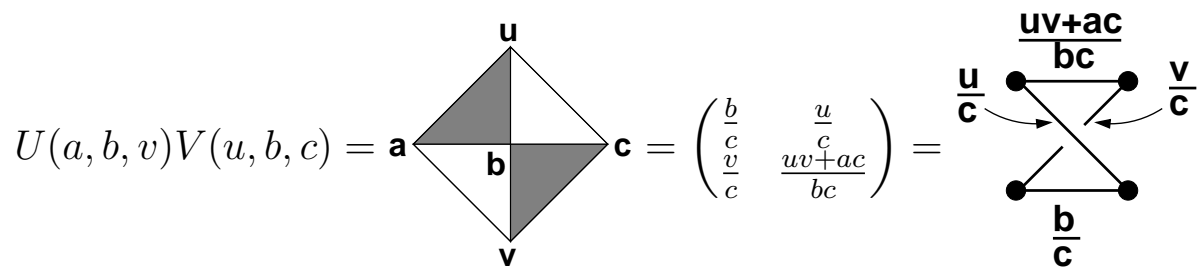


Note that as the arguments are generic, the quantity $u v+a c$ does not vanish like in the $U V$ diamond of (5.32). As the network for $N(k-r-2, k-1)$ is a square, we have a decomposition of the form (say for $r$ even):

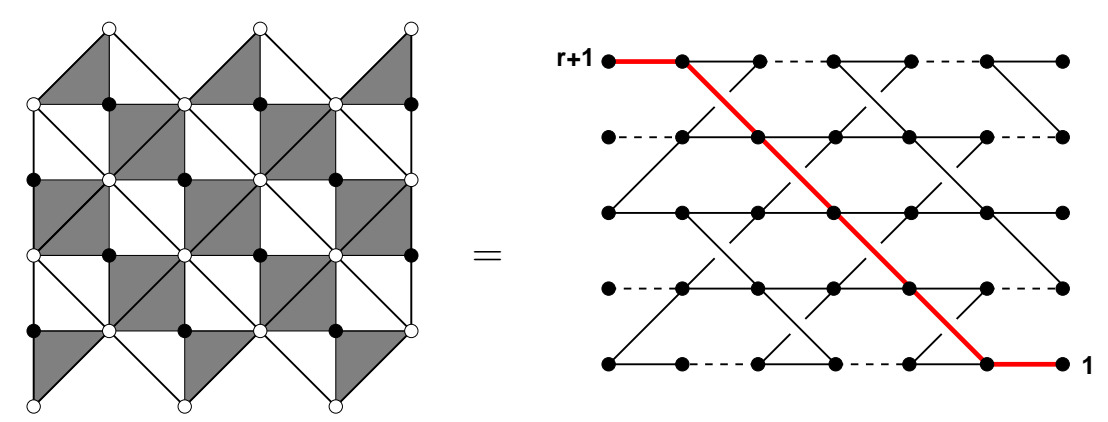

The matrix element $(r+1,1)$ corresponds to the unique path from $r+1 \rightarrow 1$, highlighted in red. The product of weights along the path is telescopic, and leaves us only with (leftmost face label $) /($ rightmost face label $)=t_{r+1, k-r-2} / t_{1, k-1}=1 / t_{1, k-1}$, as we have $t_{r+1, j}=1$ for all $j$. We conclude that $T_{1,0, k}=1$.

Case 2: $0<k-1<r+1$. In this case, $N(-k+1, k-1)=N(-k+1,0) N(0, k-1)=$ $P_{k-1} N(0, k-1)$. Then

$$
T_{1,0, k}=[N(-k+1, k-1)]_{1,1} t_{1, k-1}=[N(0, k-1)]_{2\left\lfloor\frac{k-1}{2}\right\rfloor+1,1} t_{1, k-1}
$$

by Lemma 5.18. Noting that $2\left\lfloor\frac{k-1}{2}\right\rfloor+1=k$, it is easy to see that, again, a unique path contributes to this, as the paths $k \rightarrow 1$ only "see" the lower triangle part of the network, with vertices $(i, j)=(1,0),(1, k-1),(k, 0)$, represented below:

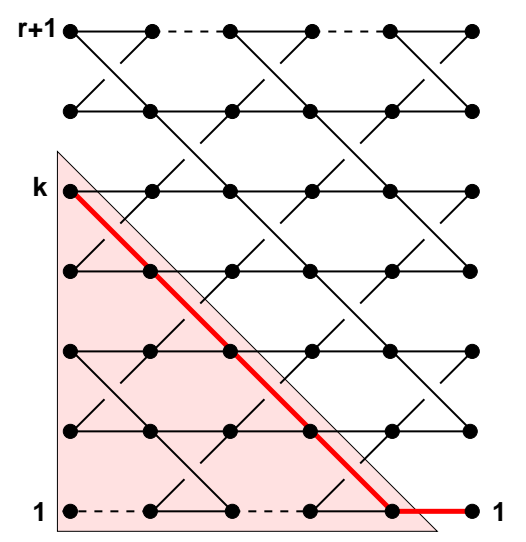

The total weight of this path is equal to (leftmost face label) $/($ rightmost face label $)=$ $t_{0, k} / t_{1, k-1}=1 / t_{1, k-1}$, which implies $T_{1,0, k}=1$.

Lemma 5.23. The solutions of the unrestricted $A_{r} T$-system of type (i) with initial conditions $X\left(\mathbf{t}^{+}\right)$have the property that $T_{1,-j, k}=0$ for all $j \in[1, r]$

Proof. Writing

$$
T_{1,-j, k}=[N(-j-k+1, k-j-1)]_{1,1} t_{1, k-j-1},
$$




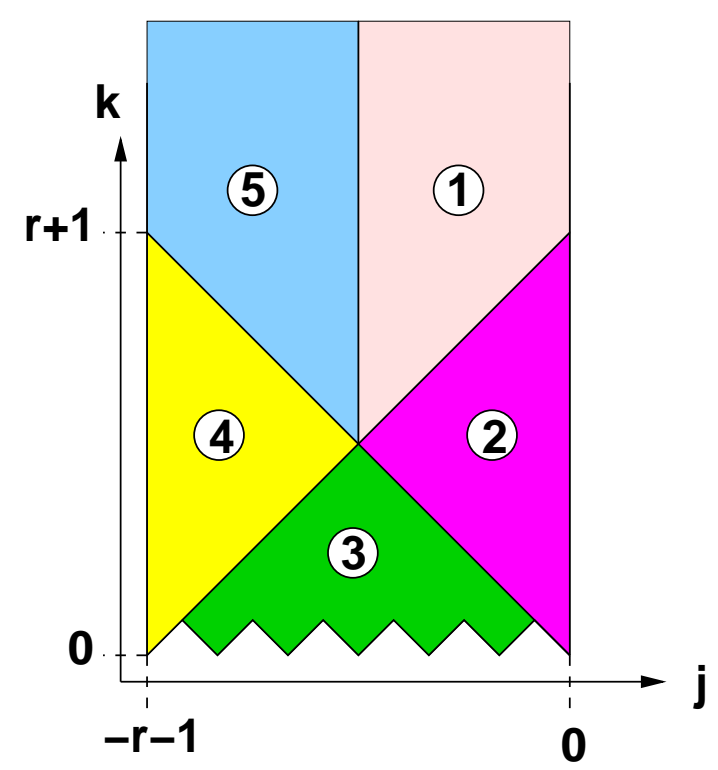

Figure 7: The five regions in $(j, k)$ plane for the proof of $T_{1,-j, k}=0$.

there are five regions for the point $(-j, k)$, which are depicted in Fig.7. Region 1: $1 \leqslant j \leqslant \frac{r+1}{2}$ and $k \geqslant r+1-j$. In this case,

$$
\begin{aligned}
N(-j-k+1, k-j-1) & =N(-j-k+1,-r-1) N(-r-1,0) N(0, k-j-1) \\
& =P N(k+j-r-2, k-j-1),
\end{aligned}
$$

where we have used Lemma 5.21. This yields

$$
T_{1,-j, k}=[N(k+j-r-2, k-j-1)]_{r+1,1} t_{1, k-j-1} .
$$

The network for $N(k+j-r-2, k-j-1)$, once decomposed into $V U$ diamonds as above, looks like:
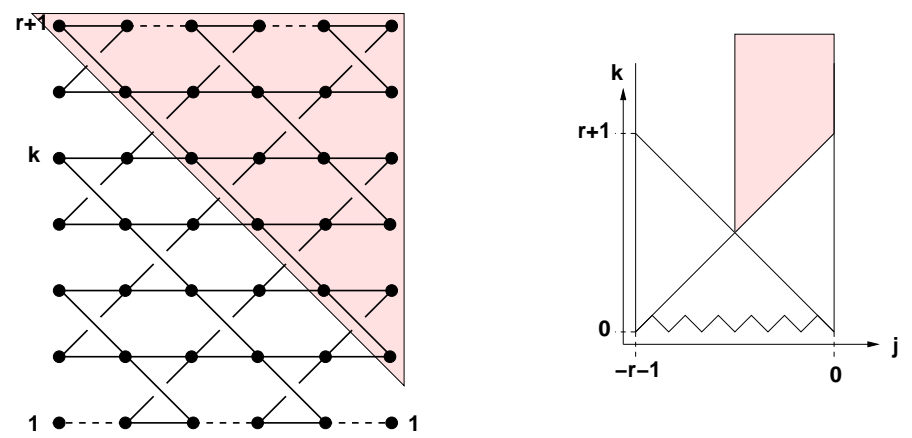

where we have represented a typical network in the diamond chip representation, and the corresponding region 1 in $(j, k)$ space. We have shaded the range of the paths from $r+1$. The network is in a rectangle of width strictly smaller than its height (size $(r+1-2 j) \times$ $(r+1))$, hence there are no path joins $r+1 \rightarrow 1$. Therefore $T_{1,-j, k}=0$.

Region 2: $r+1-j>k \geqslant j$. Since

$$
N(-j-k+1, k-j-1)=N(-j-k+1,0) N(0, k-j-1)=P_{j+k-1} N(0, k-j-1),
$$


we have

$$
T_{1,-j, k}=[N(0, k-j-1)]_{2\left\lfloor\frac{j+k-1}{2}\right\rfloor+1,1} t_{1, k-j-1} .
$$

The width of the network is $k-j-1<2\left\lfloor\frac{j+k+1}{2}\right\rfloor+1=k+j$, hence no path contributes, and $T_{1,-j, k}=0$.

Region 3: $0 \leqslant k<j$ and $k<r+1-j$. As $-r-1<-j-k+1<k-j-1<0$, we may use the matrix $N(-k-j+1, k-j-1)(\{a\})$ of the regularized network, with labels (5.22). The paths $1 \rightarrow 1$ only see the triangle shaded in the typical configuration below:
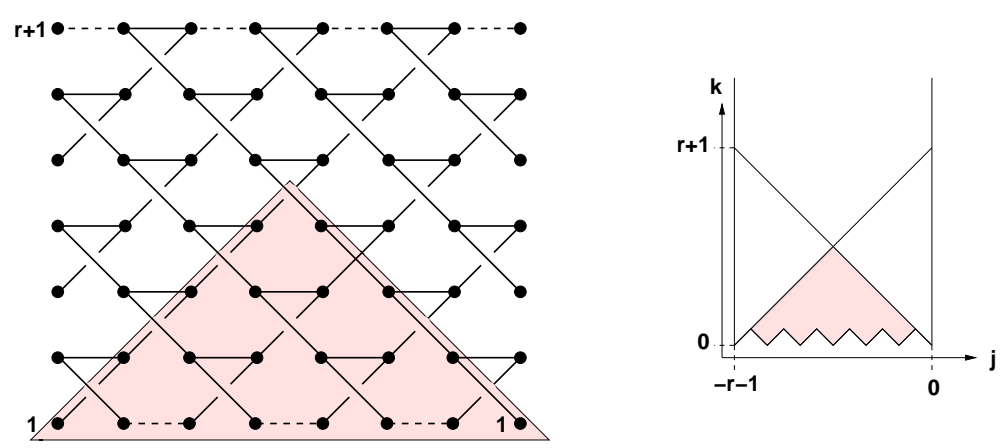

and it is clear that no path can go from $1 \rightarrow 1$, hence

$$
T_{1,-j, k}=[N(-k-j+1, k-j-1)]_{1,1} t_{0, k-j-1}=0 .
$$

Region 4: $r+1-j \leqslant k \leqslant j$. We have the decomposition $N(-k-j+1, k-j-1)=N(-k-$ $j+1,-r-1) N(-r-1, k-j-1)$. As before, we may use the matrix $N(-r-1, k-j-1)(\{a\})$ of the regularized network, with labels (5.22). In the square decomposition, the complete network looks like:
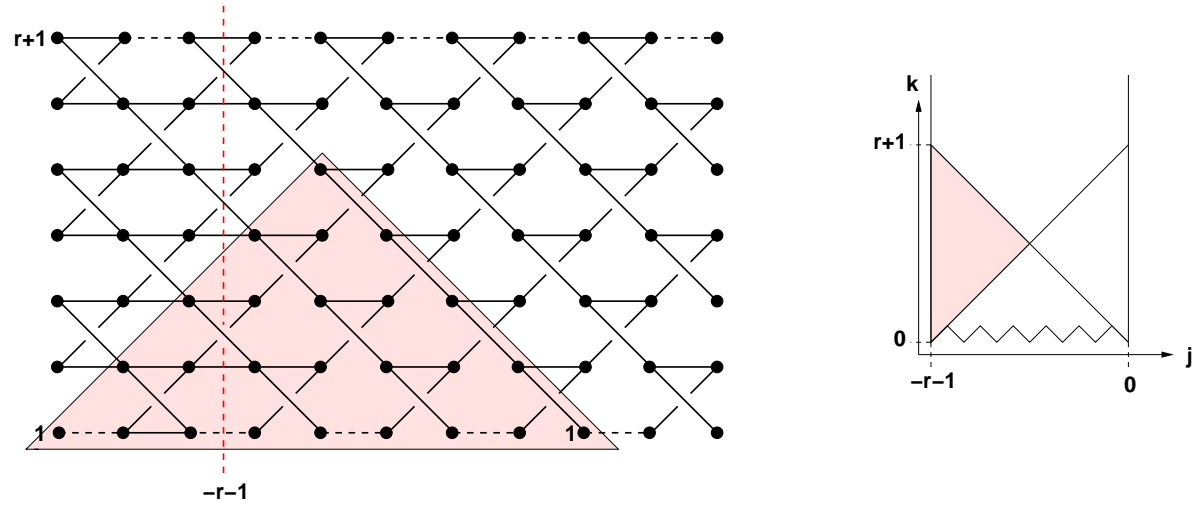

and there are no paths $1 \rightarrow 1$, as in region 3 .

Region 5: $r+1>j>\frac{r+1}{2}$ and $k>j$. We have $N(-j-k+1, k-j-1)=$ $N(-j-k+1,-r-1) P N(0, k-j-1)=N(-j-k+1, j-k-r) P$, so that $T_{1,-j, k}=$ $[N(-j-k+1, j-k-r)]_{1, r+1}$. Again, the width of the network is $2 j-r-1 \leqslant r-1$, hence there is no path from $1 \rightarrow r+1$, and $T_{1,-j, k}=0$. 

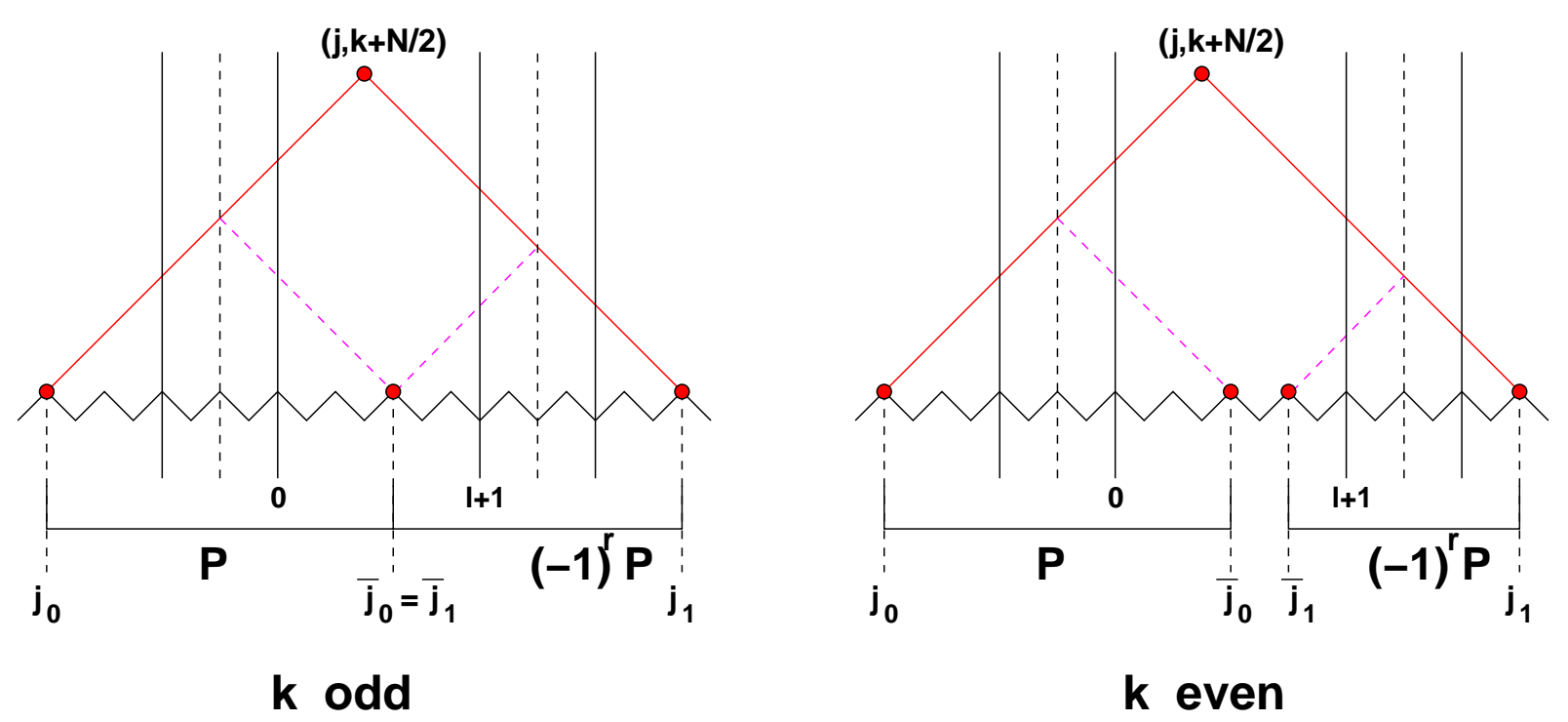

Figure 8: The projection $\left[j_{0}, j_{1}\right]$ of $(1, j, k+N / 2)$ onto $\mathbf{k}$ is shown for $k$ odd and even respectively. For $k$ odd, $j_{0}=k-N / 2, j_{1}=k+N / 2$ and the reflections $\bar{j}_{0}=\bar{j}_{1}=\ell+1-k$ coincide. For $k$ even, we have $j_{0}=k-N / 2+1, j_{1}=k+N / 2-1$ and the reflections are $\bar{j}_{0}=\ell-k$ and $\bar{j}_{1}=\ell+2-k$. In both cases, we have indicated the network matrices corresponding to the various segments.

\subsection{Proof of the periodicity Theorem 1.4}

Let $T_{i, j, k}$ be the solution of the $\ell$-restricted $A_{r} T$-system (iv). Using Theorem 5.8, it is equal to the solution $T_{i, j, k}$ of the unrestricted $A_{r} T$-system (i) subject to initial conditions $X\left(\mathbf{t}^{[1, \ell]}\right)$ on $\mathbf{k}_{0}$. We can also use the initial conditions on any integer translate $\mathbf{k}_{0}+2 m$, $m \in \mathbb{Z}$, of this surface. Let $N=2(\ell+r+2)$. Due to the determinant formula (1.8) it is sufficient to consider $i=1$. As in the $A_{1}$ case, we prove the more general half-periodicity theorem.

Theorem 5.24. The solution of the $\ell$-restricted $T$-system satisfies $T_{1, j, k+\frac{N}{2}}=T_{r, \ell+1-j, k}$ for all $k \in \mathbb{Z}, j \in[1, \ell]$ such that $j+k+\ell+r$ is odd.

Proof. We use the network solution of Theorem 2.4. Choose the surface $\mathbf{k}=2 m+\mathbf{k}_{0}$ to be the unique translation of $\mathbf{k}_{0}$ passing through the point $(r, \ell+1-j, k)$. The integer $m$ is fixed by requiring $k-2 m=k_{r, \ell+1-j}^{(0)}$, and

$$
k_{x, y}= \begin{cases}k-(x+y+1 \bmod 2), & k \text { odd } \\ k+1-(x+y+1 \bmod 2), & k \text { even }\end{cases}
$$

The corresponding initial conditions are $T_{x, y, k_{x, y}}=t_{x, y}$, where $t_{x, y}$ are initial conditions of type $\mathbf{t}^{[1, \ell]}$.

Figure 8 shows the projection of the point $\left(1, j, k+\frac{N}{2}\right)$ onto $\mathbf{k}$ in the cases when $k$ is even and odd. Let $\epsilon=1-k \bmod 2$. Then

$$
T_{1, j, k+\frac{N}{2}}=N\left(j-\frac{N}{2}+\epsilon, j+\frac{N}{2}-\epsilon\right)_{1,1} t_{1, j+\frac{N}{2}-\epsilon} .
$$


When $k$ is odd, we write

$$
\begin{aligned}
N\left(j-\frac{N}{2}, j+\frac{N}{2}\right)= & N\left(j-\frac{N}{2}, \ell+1-j\right) N\left(\ell+1-j, j+\frac{N}{2}\right) \\
= & N(j-(\ell+r+2),-r-1) N(-r-1,0) N(0, \ell+1-j) \\
& \times N(\ell+1-j, \ell+1) N(\ell+1, \ell+r+2) N(\ell+r+2, j+\ell+r+2) \\
= & P \times(-1)^{r} P=(-1)^{r} \mathbb{I}
\end{aligned}
$$

where we have used Lemma 5.21. Using $t_{1, j+\frac{N}{2}}=(-1)^{r} t_{r, \ell+1-j}$,

$$
T_{1, j, k+\frac{N}{2}}=(-1)^{r} \mathbb{I}_{1,1}(-1)^{r} t_{r, \ell+1-j}=T_{r, \ell+1-j, k} .
$$

When $k$ even, the splitting yields analogously:

$$
\begin{aligned}
N(j- & \left.\frac{N}{2}+1, j+\frac{N}{2}-1\right) \\
= & N\left(j-\frac{N}{2}+1, \ell-j\right) N(\ell-j, \ell+2-j) N\left(\ell+2-j, j+\frac{N}{2}-1\right) \\
= & N(j-(\ell+r+1),-r-1) N(-r-1,0) N(0, \ell-j) N(\ell-j, \ell+2-j) \\
& \times N(\ell+2-j, \ell+1) N(\ell+1, \ell+r+2) N(\ell+r+2, j+\ell+r+1) \\
= & P N(\ell-j, \ell+2-j)(-1)^{r} P
\end{aligned}
$$

and we get

$$
T_{1, j, k+\frac{N}{2}}=(-1)^{r} N(\ell-j, \ell+2-j)_{r+1, r+1} t_{1, j+r+\ell+1}=N(\ell-j, \ell+2-j)_{r+1, r+1} t_{r, \ell+2-j} .
$$

As $k$ is even, we have $\ell-j=r+1 \bmod 2$, and the partition function $N(\ell-j, \ell+2-j)_{r+1, r+1}$ only depends on the top part of the network matrix, namely the $V U$ diamond:

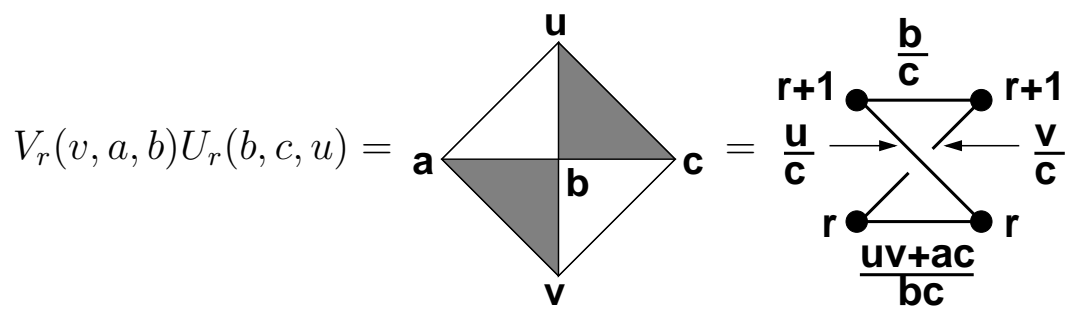

with $b=t_{r, \ell+1-j}$ and $c=t_{r, \ell+2-j}$. Therefore, $N(\ell-j, \ell+2-j)_{r+1, r+1}=t_{r, \ell+1-j} / t_{r, \ell+2-j}$, and

$$
T_{1, j, k+\frac{N}{2}}=t_{r, \ell+1-j} / t_{r, \ell+2-j} \times t_{r, \ell+2-j}=T_{1, r, \ell+1-j}
$$

Corollary 5.25. The solution of the $\ell$-restricted $A_{r} T$-system satisfies the following halfperiodicity relation:

$$
T_{i, j, k+\frac{N}{2}}=T_{r+1-i, \ell+1-j, k} \quad(i \in[1, r], j \in[1, \ell], k \in \mathbb{Z})
$$

with $N=2(\ell+r+2)$. 
Proof. Lemma 1.8 gives

$$
T_{i, j, k+\frac{N}{2}}=\operatorname{det}_{1 \leqslant a, b \leqslant i}\left(T_{1, j+a-b, k+\frac{N}{2}+a+b-i-1}\right)=\operatorname{det}_{1 \leqslant a, b \leqslant i}\left(T_{r, \ell+1-j-a+b, k+a+b-i-1}\right) .
$$

Using the Desnanot-Jacobi identity (1.9) it is possible to write solutions $T_{i, j, k}$ with $i<r$ in terms of $(r+1-i) \times(r+1-i)$ determinants of the $T_{r, j^{\prime}, k^{\prime}}$ s,

$$
T_{i, j, k}=\operatorname{det}_{1 \leqslant a, b \leqslant r+1-i}\left(T_{r, j+a-b, k+a+b-r-2+i}\right) .
$$

Therefore,

$$
T_{r+1-i, \ell+1-j, k}=\operatorname{det}_{1 \leqslant a, b \leqslant i}\left(T_{r, \ell+1-j+a-b, k+a+b-i-1}\right),
$$

Comparing this with (5.45), and noting that the transposed matrix has the same determinant yields (5.44).

In particular, we have that $T_{r, j, k+\frac{N}{2}}=T_{1, \ell+1-j, k}$. Combining this with Theorem 5.24, we deduce that $T_{1, j, k+N}=T_{1, j, k}$, and therefore $T_{i, j, k+N}=T_{i, j, k}$. This completes the proof of the periodicity.

\subsection{Positivity: Proof of Theorems 5.9 and 1.5}

Theorem 5.9 is the claim that solutions of the $A_{r} T$-system of type (ii) are Laurent polynomials with non-negative integer coefficients of the initial data $\mathbf{t}$.

Lemma 5.26. The solutions $T_{1, j, k}$ of the half-space $A_{r} T$-system of type (ii) with initial conditions $X^{+}(\mathbf{t})$ (5.4) on the surface $\mathbf{k}_{0}=\{(i, j, i+j \bmod 2): i \in[1, r], j>0\}$ are Laurent polynomials of $\left\{t_{i, j}: i \in[1, r], j>0\right\}$ with non-negative integer coefficients.

Proof. First, use Theorem 5.6 to identify $T_{1, j, k}$ as the solution of the $A_{r} T$-system of type (i) with initial data $\mathbf{t}^{+}$. Consider the projection of $(1, j, k)$ onto $\mathbf{k}_{0}$, with minimum $j_{0}$ and maximum $j_{1}$. We will show that the partition function for paths starting at $\left(1, j_{0}\right)$ and ending at $\left(1, j_{1}\right)$ on the network $N\left(j_{0}, j_{1}\right)$, and with weights $\mathbf{t}^{+}$, is equal to the partition function from $\left(i_{0}, \bar{j}_{0}\right)$ to $\left(1, j_{1}\right)$ on the network $N\left(\bar{j}_{0}, j_{1}\right)$, for some $i_{0} \in[1, r+1]$ and $\bar{j}_{0} \in\left[0, j_{1}\right]$. The latter portion $N\left(\bar{j}_{0}, j_{1}\right)$ of the network has only positive weights from the set $\mathbf{t}^{+}$, hence positivity follows.

The formula for $\left(i_{0}, \bar{j}_{0}\right)$ depends on the value of $j_{0}$. Three cases may occur:

- $j_{0} \geqslant 0:\left(i_{0}, \bar{j}_{0}\right)=\left(1, j_{0}\right)$. The solution is identical to that of the unrestricted $A_{r}$ $T$-system of type (i), and positivity follows from Theorem 1.3.

- $j_{0} \leqslant-r-1:\left(i_{0}, \bar{j}_{0}\right)=\left(r+1,-r-1-j_{0}\right)$. This is a consequence of the collapse relations on the network solution,

$$
T_{1, j, k}=\left[N\left(j_{0}, j_{1}\right)\right]_{1,1} t_{1, j_{1}}=\left[P N\left(\bar{j}_{0}, j_{1}\right)\right]_{1,1} t_{1, j_{1}}=\left[N\left(\bar{j}_{0}, j_{1}\right)\right]_{r+1,1} t_{1, j_{1}},
$$

where $\bar{j}_{0}=-r-1-j_{0}$ (see Figure 9 for an illustration). 


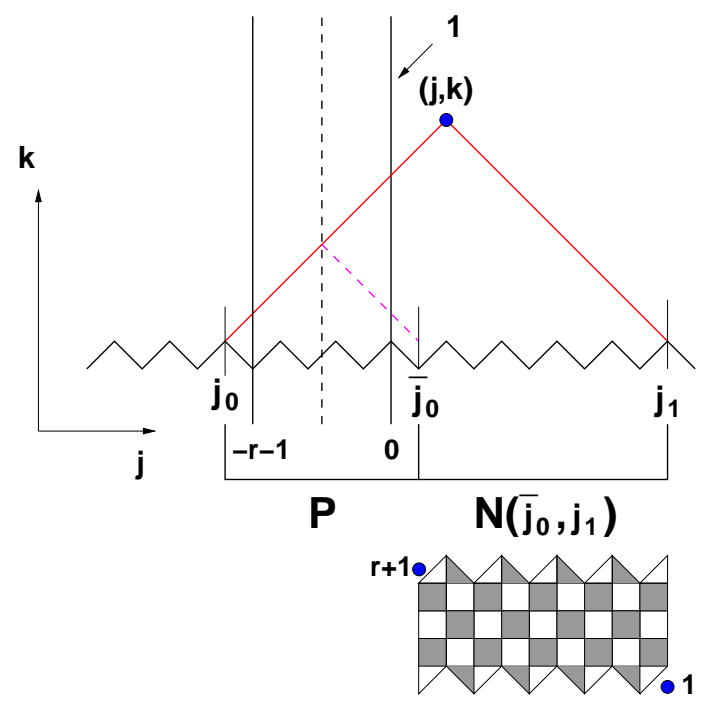

$j_{0} \leqslant-r-1$

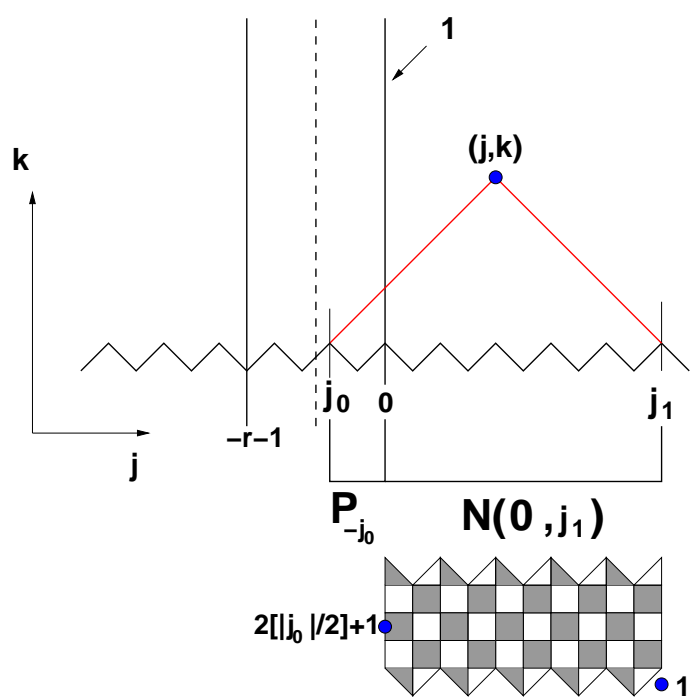

$-r \leqslant j_{0} \leqslant-1$

Figure 9: The cases (ii) $j_{0} \leqslant-r-1$ and (iii) $-r \leqslant j_{0} \leqslant-1$ for the position of the minimum of the projection of $(1, j, k)$ onto $\mathbf{k}_{0}$. We have represented in both cases the resulting network and the position of the starting and ending point of the paths whose partition function produces $T_{1, j, k} / T_{1, j_{1}, k_{j_{1}}^{(0)}}$.

- $-r \leqslant j_{0} \leqslant-1:\left(i_{0}, \bar{j}_{0}\right)=\left(2\left\lfloor\frac{\left|j_{0}\right|}{2}\right\rfloor+1,0\right)$. This follows by applying Lemma 5.18 to the network solution:

$$
T_{1, j, k}=\left[N\left(j_{0}, j_{1}\right)\right]_{1,1} t_{1, j_{1}}=\left[P_{-j_{0}} N\left(0, j_{1}\right)\right]_{1,1} t_{1, j_{1}}=\left[N\left(0, j_{1}\right)\right]_{2\left\lfloor\frac{\left|j_{0}\right|}{2}\right\rfloor+1,1} t_{1, j_{1}} .
$$

(see the right of Figure 9 for an illustration).

Corollary 5.27. The solutions $T_{i, j, k}$ of the half-space $A_{r} T$-system of type (ii) are nonnegative Laurent polynomials of the initial data $\left\{t_{i, j}: i \in[1, r], j>0\right\}$, assigned at points on $\mathbf{k}_{0}$ with $j>0$.

Proof. We use the determinant formula (1.8) for $T_{i, j, k}$. Let $j_{0}(b)=j-k+i-2(b-1)$, $b=1,2, \ldots, i$ and $j_{1}(a)=j+k-i+2(a-1), a=1,2, \ldots, i$ be respectively the minima and the maxima of the projections of the $i^{2}$ points $(1, j+a-b, k+a+b-i-1)$ involved in the formula. From the proof of the previous lemma, the quantity $Z_{a, b}=$ $T_{1, j+a-b, k+a+b-i-1} / t_{1, j_{1}(a)}$ is the partition function for paths on $N\left(0, j_{1}\right)$, ending at position $\left(1, j_{1}(a)\right)$ and starting at position $\left(i_{0}(b), \bar{j}_{0}(b)\right)$, defined as the pair $\left(i_{0}, \bar{j}_{0}\right)$ of Lemma 5.26 for $j_{0}=j_{0}(b)$. The Lindström-Gessel-Viennot Theorem [19, 20] gives an interpretation of the determinant $T_{i, j, k} / \prod_{a=1}^{i} t_{1, j_{1}(a)}=\operatorname{det}_{1 \leqslant a, b \leqslant i}\left(Z_{a, b}\right)$, as the partition function of $i$ nonintersecting paths on the network of $N\left(0, j_{1}\right)$ with $i$ starting points $\left(i_{0}(b), \bar{j}_{0}(b)\right)(b \in[1, i])$, 
and with $i$ ending points at positions $\left(1, j_{1}(a)\right)(a \in[1, i])$. We deduce the positive Laurent property from the positivity of weights.

We have the analogous result for the left half-space $T$-system:

Lemma 5.28. The solutions of the left half-space $A_{r}$ T-system (iii) are Laurent polynomials with non-negative integer coefficients of the initial data $\left\{t_{i, j}: i \in[1, r], j \leqslant \ell\right\}$ assigned at the points of the surface $\mathbf{k}_{0}$ with $j \leqslant \ell$.

Proof. The proof is identical to that of Lemma 5.26 and Corollary 5.27. We start from Theorem 5.7 to identify $T_{1, j, k}$ as the solution of the $A_{r} T$-system of type (i) with initial data $\mathbf{t}^{-}$. Consider the projection of $(1, j, k)$ onto $\mathbf{k}_{0}$, with minimum $j_{0}$ and maximum $j_{1}$. We will show that the product of $t_{1, j_{1}}$ with the partition function for paths starting at $\left(1, j_{0}\right)$ and ending at $\left(1, j_{1}\right)$ on the network $N\left(j_{0}, j_{1}\right)$, and with weights $\mathbf{t}^{-}$, is equal to the product of $t_{\lambda_{1}, \bar{j}_{1}}$ with the partition function from $\left(1, j_{0}\right)$ to $\left(i_{1}, \bar{j}_{1}\right)$ on the network $N\left(j_{0}, \bar{j}_{1}\right)$, for some $\lambda_{1} \in[1, r], i_{1} \in[1, r+1]$ and $\bar{j}_{1} \in\left[j_{0}, \ell+1\right]$. The latter portion $N\left(j_{0}, \bar{j}_{1}\right)$ of the network has only positive weights from the set $\mathbf{t}^{-}$, hence positivity follows.

The formula for $\left(\lambda_{1}, i_{1}, \bar{j}_{1}\right)$ reads as follows. Three cases may occur:

- $j_{1} \leqslant \ell+1:\left(\lambda_{1}, i_{1}, \bar{j}_{1}\right)=\left(1,1, j_{1}\right)$. The solution is identical to that of the unrestricted $A_{r} T$-system of type (i), and positivity follows from Theorem 1.3.

- $j_{1} \geqslant \ell+r+2:\left(\lambda_{1}, i_{1}, \bar{j}_{1}\right)=\left(r, r+1,2 \ell+r+3-j_{1}\right)$. This is a consequence of the collapse relations on the network solution, and of the symmetries of $\mathbf{t}^{-}$:

$$
T_{1, j, k}=\left[N\left(j_{0}, j_{1}\right)\right]_{1,1} t_{1, j_{1}}=\left[N\left(j_{0}, \bar{j}_{1}\right)(-1)^{r} P\right]_{1,1}(-1)^{r} t_{r, \bar{j}_{1}}=\left[N\left(j_{0}, \bar{j}_{1}\right)\right]_{r+1,1} t_{r, \bar{j}_{1}},
$$

where we have used Lemma 5.19 and $\bar{j}_{1}=2 \ell+r+3-j_{1}$.

- $\ell+1<j_{1}<\ell+r+2:\left(\lambda_{1}, i_{1}, \bar{j}_{1}\right)=\left(1, a_{\ell}\left(j_{1}-\ell-1\right), \ell+1\right)$, with $a_{\ell}(x)$ is as in (5.40). This follows by applying Lemma 5.20 to the (regularized) network solution:

$$
T_{1, j, k}=\lim _{b_{1}, \ldots, b_{r} \rightarrow 0}\left[N\left(j_{0}, \ell+1\right) \tilde{P}_{j_{1}-\ell-1}(\{b\})\right]_{1,1} b_{1, j_{1}}=\left[N\left(j_{0}, \ell+1\right)\right]_{1, a_{\ell}\left(j_{1}-\ell-1\right)},
$$

and noting that $1=t_{1, \ell+1}$.

The equivalent of Corollary 5.27 follows from interpreting à la Gessel-Viennot the quantity $T_{i, j, k} / \prod_{a=1}^{i} t_{\lambda_{1}(a), \bar{j}_{1}(a)}$, as the partition function for $i$ non-intersecting paths on the network that start at $\left(1, j_{0}(b)\right)(b \in[1, i])$ and end at $\left(i_{1}(a), \bar{j}_{1}(a)\right)(a \in[1, i])$. Positivity follows.

Theorem 1.5 follows from:

Lemma 5.29. The solutions of the $A_{r} T$-system with initial data of type $\mathbf{t}^{[1, \ell]}$ are Laurent polynomials with non-negative integer coefficients of the variables $\left\{t_{i, j}: i \in[1, r], j \in\right.$ $[1, \ell]\}$, assigned along the points of $\mathbf{k}_{0}$ with $0<j \leqslant \ell$. 
Proof. We start by proving the property for $T_{1, j, k}$. The half-periodicity property of Theorem 5.24 allows us to restrict to points $(1, j, k)$ with $0 \leqslant k \leqslant \frac{N}{2}$.

Let $j_{0}$ and $j_{1}$ be the minimum and maximum of the projection of $(1, j, k)$ onto $\mathbf{k}_{0}$. Using the definitions of $i_{0}, \bar{j}_{0}, \lambda_{1}, i_{1}, \bar{j}_{1}$ given in the proofs of Lemmas 5.26 and 5.28, we will show that the network partition function for paths from $\left(1, j_{0}\right)$ to $\left(1, j_{1}\right)$, multiplied by $t_{1, j_{1}}$ is equal to $t_{\lambda_{1}, \bar{j}_{1}}$ times the partition function of paths from $\left(i_{0}, \bar{j}_{0}\right)$ to $\left(i_{1}, \bar{j}_{1}\right)$ where $0 \leqslant \bar{j}_{0} \leqslant \bar{j}_{1} \leqslant \ell+1$. The weights in this region of $\mathbf{t}^{[1, \ell]}$ are all positive, hence so is the partition function. Define the following subsets of $\mathbb{Z}$ :

$$
\begin{gathered}
A=[0, \ell+1], \quad B=[-\ell-r-2,-r-1], \quad C=[-r,-1], \\
D=[r+\ell+2, r+2 \ell+3], \quad E=[\ell+2, r+\ell+1] .
\end{gathered}
$$

When $j_{0}$ or $j_{1} \in A$, the solution is identical to that of Theorem 1.3, Lemma 5.9 or Corollary 5.28 , in which positivity has been proven. There are four remaining cases.

- $\left(j_{0}, j_{1}\right) \in B \times D$ : We use collapse relations on both sides:

$$
T_{1, j, k}=\left[P N\left(\bar{j}_{0}, \bar{j}_{1}\right)(-1)^{r} P\right]_{1,1} t_{1, j_{1}}=\left[N\left(\bar{j}_{0}, \bar{j}_{1}\right)\right]_{r+1, r+1} t_{r, \bar{j}_{1}}
$$

- $\left(j_{0}, j_{1}\right) \in B \times E$ : We use collapse relations on the left, and Lemma 5.20 on the right:

$$
T_{1, j, k}=\lim _{b_{1}, \ldots, b_{r} \rightarrow 0}\left[P N\left(\bar{j}_{0}, \ell+1\right) \tilde{P}(\{b\})_{j_{1}-\ell-1}\right]_{1,1} b_{1, j_{1}}=\left[N\left(\bar{j}_{0}, \ell+1\right)\right]_{r+1, a_{\ell}\left(j_{1}-\ell-1\right)}
$$

- $\left(j_{0}, j_{1}\right) \in C \times D$ : We use collapse relations on the right, and Lemma 5.18 on the left:

$$
T_{1, j, k}=\left[P_{-j_{0}} N\left(0, \bar{j}_{1}\right)(-1)^{r} P\right]_{1,1} t_{1, j_{1}}=\left[N\left(0, \bar{j}_{1}\right)\right]_{a_{1}\left(\left|j_{0}\right|\right), r+1} t_{r, \bar{j}_{1}}
$$

- $\left(j_{0}, j_{1}\right) \in C \times E$ : We use Lemma 5.18 on the left and Lemma 5.20 on the right:

$$
T_{1, j, k}=\lim _{b_{1}, \ldots, b_{r} \rightarrow 0}\left[P_{-j_{0}} N(0, \ell+1) \tilde{P}_{j_{1}-\ell-1}(\{b\})\right]_{1,1} b_{1, j_{1}}=[N(0, \ell+1)]_{a_{1}\left(\left|j_{0}\right|\right), a_{\ell}\left(j_{1}-\ell-1\right)}
$$

To summarize, in all cases $T_{1, j, k}$ is expressed in terms of the partition function for paths on the same network but with different starting and ending positions, depending on the values of $j$ and $k$. We may now apply the Lindström-Gessel-Viennot theorem $[19,20]$ to the determinant expression of Lemma 1.2. Let $j_{0}(b)=j-k+i-2(b-1)$ and $j_{1}(a)=j+k-i+2(a-1)$ be the minima and maxima of the projections of the points $\left(1, j^{\prime}, k^{\prime}\right)$ involved in the determinant. We interpret the quantity $T_{i, j, k} / \prod_{a=1}^{i} t_{\lambda_{1}(a), \bar{j}_{1}(a)}$, as the partition function for $i$ non-intersecting paths on the network, starting at $\left(i_{0}(b), j_{0}(b)\right)$ $(b \in[1, i])$ and ending at $\left(i_{1}(a), \bar{j}_{1}(a)\right)(a \in[1, i])$. This proves positivity, as all the path weights are positive Laurent monomials of the initial data $t_{i, j}$ in the positive part of $\mathbf{t}^{[1, \ell]}$.

Typically, depending on $i, j, k$, and as $i \leqslant r$, we may have at worst some of the minima in $A$ and the rest in $C$, or some in $B$ and the rest in $C$, and similarly for the maxima, either in $A \cup D$ or in $A \cup E$. We illustrate this in Fig. 10 for $r=6$ and 


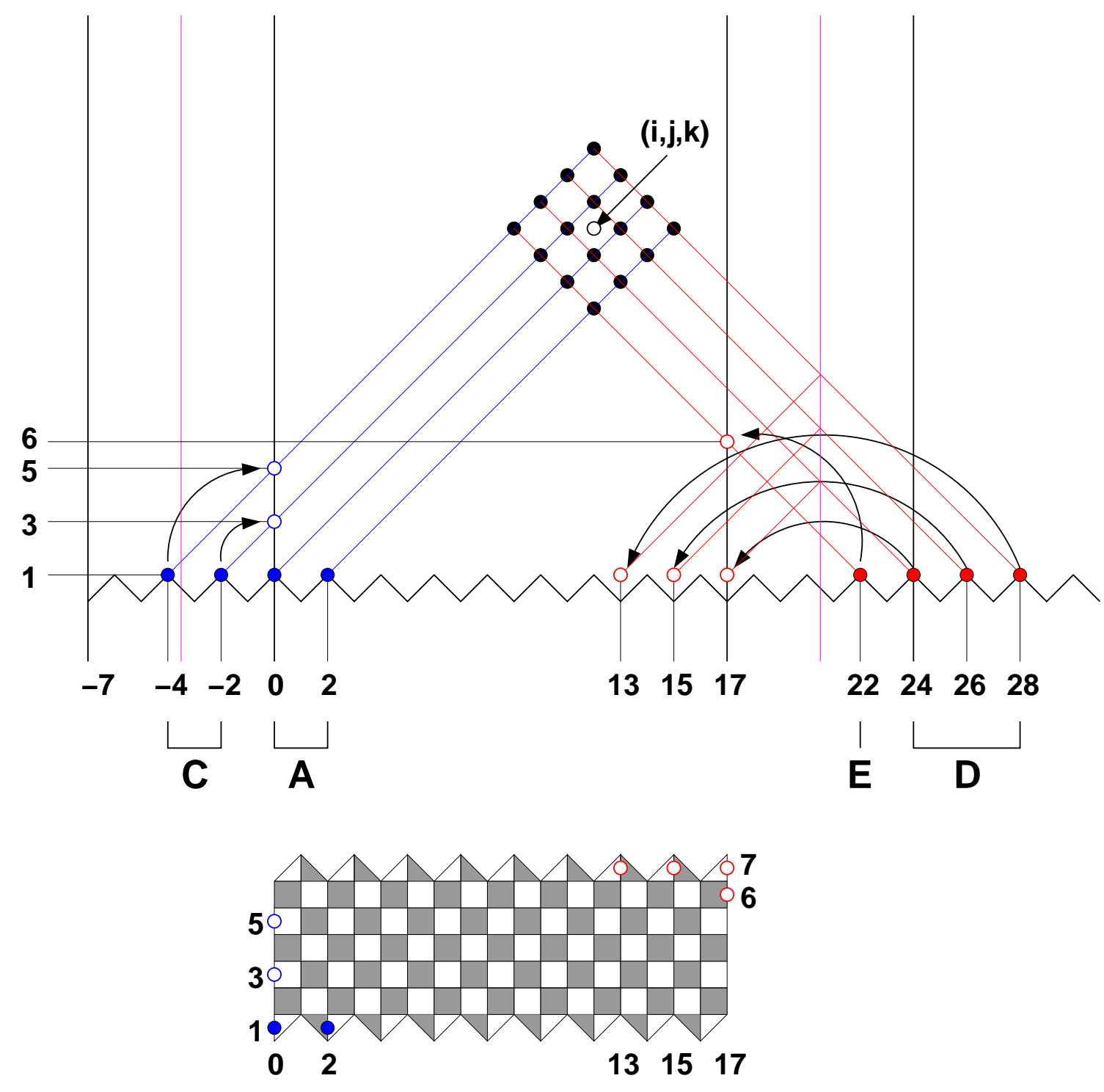

Figure 10: A typical example of expression of $T_{i, j, k}$ as non-intersecting path partition function. Here $(i, j, k)=(4,12,14), r=6$ and $\ell=16$. We have indicated the 16 terms involved in the determinant of Lemma 1.2 by black dots, and by blue (resp. red dots) their projection minima (resp. maxima) onto $\mathbf{k}_{0}$. The corresponding starting and endpoints are indicated on the associated network picture in representation I. 

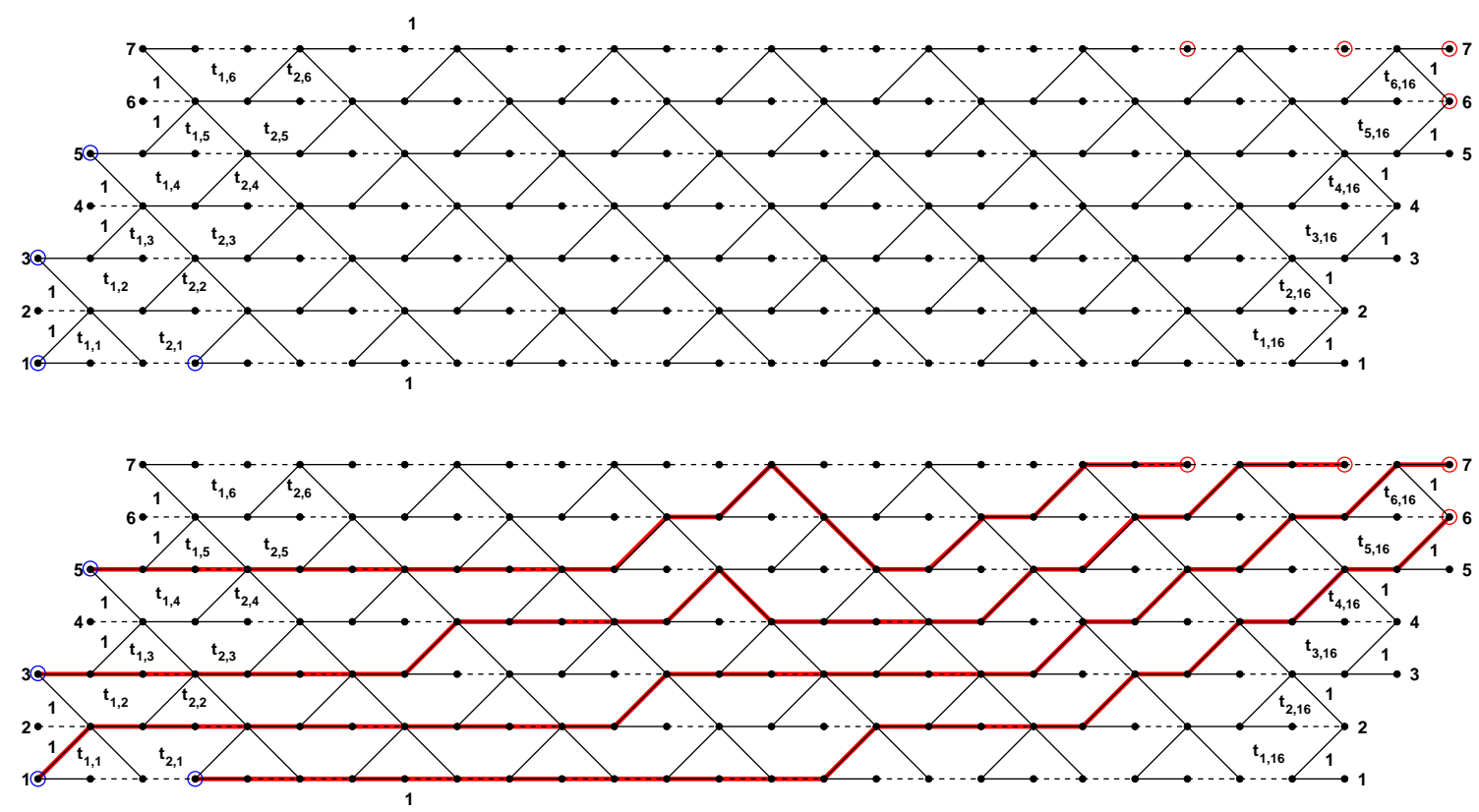

Figure 11: The pictorial representation II of the network of Fig.10, with starting points circled in blue and endpoints circled in red. We have also represented a typical configuration of non-intersecting paths on the network that contribute to $T_{4,12,14}$.

$\ell=16$, and $(i, j, k)=(4,12,14)$. In this case, $A=[0,17], B=[-24,-7], C=[-6,-1]$, $D=[24,41], E=[18,23]$. The minima $j_{0}(1)=-4, j_{0}(2)=-2$ are both in $C$ and give rise to respective starting points $(5,0),(3,0)$, while $j_{0}(3)=0, j_{0}(4)=2$ are both in $A$ and give rise to starting points $(1,0),(1,2)$. The maxima are $j_{1}(1)=22$ in $E$ giving rise to the endpoint $\left(a_{\ell}\left(j_{1}(1)-\ell-1\right), \ell+1\right)=(6,17)$, and $j_{1}(2)=24, j_{1}(3)=26, j_{1}(4)=28$, all in $D$, giving rise to the endpoints $(7,17),(7,15),(7,13)$. The network partition function for these 4 non-intersecting paths is equal to $T_{4,12,14} /\left(t_{6,13} t_{6,15}\right)$.

We have represented the corresponding network in the pictorial representation II in Fig.11, together with a typical configuration of four non-intersecting paths that contributes to $T_{4,12,14}$.

\section{Other boundary conditions}

So far, we have used the network solution of the $T$ systems of type $A$ to find expressions for their solutions for wall-type boundary conditions. In particular, in the $\ell$-restricted case, this gives a combinatorial proof of Zamolodchikov's periodicity conjecture.

There are other interesting types of boundary conditions on the $T$-system, and we comment on some of them below. 


\subsection{Friezes}

The so-called $S L_{2}$ frieze patterns $[3,1]$ are known to obey the $A_{1} T$-system relation. In [1], special boundaries were considered, coded by affine Dynkin diagrams. In particular, the case of the affine Dynkin diagram $\tilde{A}_{\ell}$ with an acyclic orientation corresponds to the $A_{1} T$-system with a periodic initial data path $\mathbf{k}$, such that $k_{j+\ell}=k_{j}+m$ for some fixed integer $m$ and for all $j \in \mathbb{Z}$, and periodic initial values $\mathbf{t}$ along this path, with $t_{j+\ell}=t_{j}$ for all $j \in \mathbb{Z}$. The Laurent positivity for this case follows immediately from that of the unrestricted system. Note that the case of the ordinary Dynkin diagram $A_{\ell}$ corresponds to the $\ell$-restricted boundaries.

In the context of higher rank $T$-systems, boundary conditions coded by pairs $\left(G, G^{\prime}\right)$ of Dynkin diagrams lead to the most general periodicity conjecture of Zamolodchikov, proved in [15]. In that context, the first Dynkin diagram codes the type of $T$-system $\left(A_{r}\right.$ throughout this paper), while the second codes the particular boundary conditions $\left(A_{\ell}\right.$ for the $\ell$-restricted boundaries for instance).

In a way similar to frieze patterns, we may consider the case $\left(A_{r}, \tilde{A}_{\ell-1}\right)$, for even integers $\ell \geqslant 2$, where the $A_{r} T$-system solutions are $\ell$-periodic in the $j$ direction, with $T_{i, j+\ell, k}=T_{i, j, k}$. This is guaranteed by imposing that both the stepped surface $\mathbf{k}$ and the attached initial data $t_{i, j}$ of $(1.7)$ be $\ell$-periodic in the $j$ direction, i.e. $k_{i, j+\ell}=k_{i, j}+m_{i}$ for some fixed $m_{i}$ compatible with the stepped surface conditions of Def.1.1 and $t_{i, j+\ell}=t_{i, j}$ for all $i \in[1, r]$ and $j \in \mathbb{Z}$. The positivity of the corresponding $T$-system follows from Theorem 1.3.

We may also consider the case $\left(\tilde{A}_{r-1}, \tilde{A}_{\ell-1}\right)$ for even $r, \ell \geqslant 2$, in which the $T$-system is wrapped on a torus, by imposing that the $T$-system solutions be doubly periodic, with $T_{i+r, j, k}=T_{i, j+\ell, k}=T_{i, j, k}$ for all $i, j, k \in \mathbb{Z}$. The solutions of the corresponding system are obtained from those of the unrestricted $A_{\infty} T$-system by imposing that both the stepped surface and the initial data of (1.3) be doubly periodic as well. Positivity then follows from Theorem 1.3.

\subsection{Higher pentagram maps as $T$-system tori}

The pentagram map has been shown to relate to cluster algebra, and its solution was expressed in [10] in terms of some particular $T$-system solution. Higher versions of this map were considered by [11]. In all cases, we note that these correspond to quivers that are quotients of the $T$-system quiver by a torus, defined as follows.

Let us consider the solutions of the unrestricted $A_{\infty} T$-system, with initial data $\mathbf{t}$ along the stepped surface $\mathbf{k}_{0}$ satisfying a toric periodicity property. Let us fix $\vec{a}=\left(a_{1}, a_{2}\right)$, $\vec{b}=\left(b_{1}, b_{2}\right)$ two non-collinear vectors in $\mathbb{Z}^{2}$ and such that $a_{1}+a_{2}$ and $b_{1}+b_{2}$ are even. We impose the double periodicity property:

$$
\Theta_{\vec{a}, \vec{b}}: \quad t_{i+a_{1}, j+a_{2}}=t_{i, j} \quad t_{i+b_{1}, j+b_{2}}=t_{i, j}
$$

This is a generalization of the rectangular torus case $\left(\tilde{A}_{r-1}, \tilde{A}_{\ell-1}\right)$ described in the previous section, corresponding to $\vec{a}=(r, 0)$ and $\vec{b}=(0, \ell)$. 
In the cluster algebra identification for the $T$-system, the seed of the cluster algebra is made of a cluster and an exchange matrix, both of infinite size, as the rank is infinite. The cluster is the set of initial values $\left\{t_{i, j}\right\}$ along the stepped surface $\mathbf{k}_{0}$, and gets mutated into other initial data. The exchange matrix is coded by the quiver $Q_{0}$ with vertices $(j, i) \in \mathbb{Z}^{2}$ and oriented edges $(j, i) \rightarrow(j \pm 1, i)$, and $(j, i \pm 1) \rightarrow(j, i)$ for all $i, j \in \mathbb{Z}^{2}$ with $i+j$ even. Note that the edge configurations around even vertices $((j, i)$ with $i+j$ even) are opposite to those around odd ones $((j, i)$ with $i+j$ odd $)$. The parity conditions $\left(a_{1}+a_{2}\right.$ and $b_{1}+b_{2}$ even) guarantee that only vertices of the same parity are identified. By taking a quotient of $\mathbb{Z}^{2}$ by the lattice $\mathbb{Z} \vec{a}+\mathbb{Z} \vec{b}$, this allows to fold the corresponding infinite quiver $Q_{0}$ into a finite one $\tilde{Q}_{0}$. The example below illustrates the case $\vec{a}=(0,2)$ and $\vec{b}=(3,1)$ :
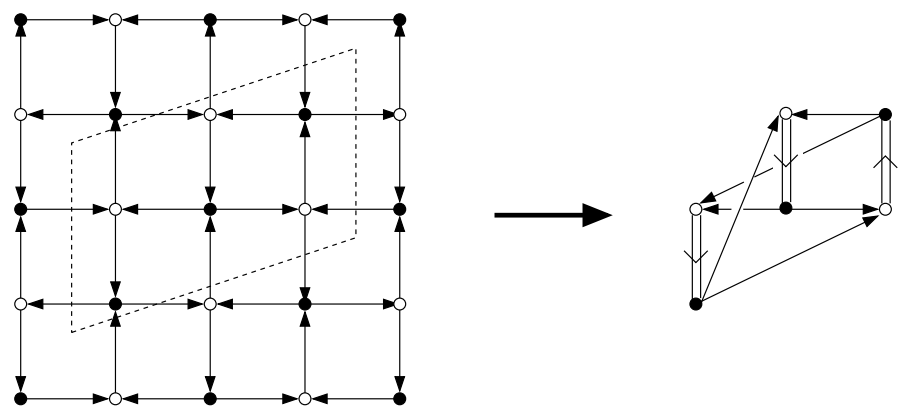

The folded system obtained by considering torus-periodic initial values and performing a quotient by $\mathbb{Z} \vec{a}+\mathbb{Z} \vec{b}$ is also part of a cluster algebra. Its mutations $\mu_{i, j}$ correspond to considering infinite compound (mutually commuting) mutations $\prod_{m, n \in \mathbb{Z}} \mu_{i+n a_{1}, m b_{1}, j+n a_{2}+m b_{2}}$ and passing to the quotient.

The quiver of the initial seed of the cluster algebra underlying the pentagram map of [10] was generalized to a quiver $Q_{k, n}$ for higher pentagram maps in [11] (the former case corresponding to $k=3$ ). The quiver $Q_{k, n}$ has two (even and odd) sets of vertices denoted by $p_{i}, q_{i}, i \in \mathbb{Z}$ with periodic identifications $p_{i+n}=p_{i}$ and $q_{i+n}=q_{i}$ which makes the quiver finite, with $2 n$ vertices. It is easy to rewrite the (infinite) quiver before identifications as that, $Q_{0}$, of the unrestricted $A_{\infty} T$-system with vertices $(j, i) \in \mathbb{Z}^{2}$ as follows:

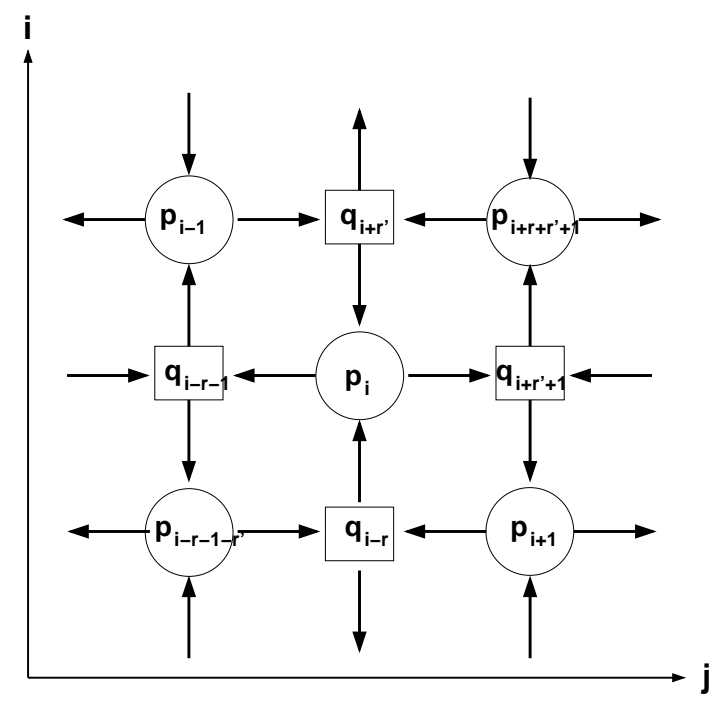


where $r, r^{\prime}$ are two non-negative integers such that $r+r^{\prime}=k-2$ and $r^{\prime}=r$ if $k$ is even, and $r^{\prime}=r+1$ otherwise, and where we have represented by circles (resp. squares) the vertices $(j, i) \in \mathbb{Z}^{2}$ with $i+j$ even (resp. odd). Note that horizontal arrows correspond to a shift $+r^{\prime}+1$ (resp. $-r-1$ ) in the indices when the arrow points to the right (resp. left), while vertical arrows correspond to a shift $+r$ (resp. $\left.-r^{\prime}\right)$ in the indices when the arrow points up (resp. down). The identifications $p_{i} \equiv p_{i+n}$ and $q_{i}=q_{i+n}$ of the two types of vertices are therefore equivalent to a double translational invariance. A simple calculation shows that it corresponds to the torus $\mathbb{Z}^{2} / \mathbb{Z} \vec{a}+\mathbb{Z} \vec{b}$, with $\vec{a}=(-(k-2)$, $k)$ and

$\vec{b}=(-n, n)$ with a fundamental domain under these translations containing $|\vec{a} \wedge \vec{b}|=2 n$ vertices. In all cases, the finite quiver $Q_{k, n}$ with $2 n$ vertices is the corresponding folding of $Q_{0}$.

\subsection{Cuts}

We may consider solutions of the $T$-system with cuts defined as follows. We consider the unrestricted $A_{\infty} T$-system, and pick a set $S$ of tetrahedra (called singular) along which the $T$-system relation is not imposed. A natural question for this system is: for which choices of $S$ and of initial conditions does the positive Laurent property hold?

In the $A_{1}$ case, $S$ is a set of diamonds of the form $\left(W_{i}, S_{i}, E_{i}, N_{i}\right)=\left(\left(j_{i}-1, k_{i}\right),\left(j_{i}, k_{i}-\right.\right.$ $\left.1),\left(j_{i}+1, k_{i}\right),\left(j_{i}, k_{i}+1\right)\right)$ for $i=1, \ldots,|S|$. The particular case of a single diamond $|S|=1$ (which we refer to as "puncture") may be solved by the techniques of the present paper. Consider an initial data surface of the form:



with the following initial conditions. Let $\epsilon_{j}=0$ if $j$ is odd, $\epsilon_{j}=-1$ if $j$ is even positive and $\epsilon_{j}=1$ if $j$ is even negative. Then the initial data assignment for the system with a puncture at $((-1,0),(0,-1),(1,0),(0,1))$ is:

$$
\begin{aligned}
T_{j, \epsilon_{j}} & = \begin{cases}a_{j} & \text { if } j>0 \\
b_{j} & \text { if } j<0\end{cases} \\
T_{0,1} & =x \\
T_{0,-1} & =y
\end{aligned}
$$

for $x, y$ some formal invertible variables. The Laurent property of the solutions is a direct consequence of Theorem 4.1. In fact, for any point above the initial data paths, the solution is a Laurent polynomial of the $a$ 's, $b$ 's and of $x$, while for any point below the initial data paths, the solution is a Laurent polynomial of the $a$ 's, $b$ 's and of $y$. More interestingly, we may consider different initial data paths containing the edges of the 
puncture, such as the example below:

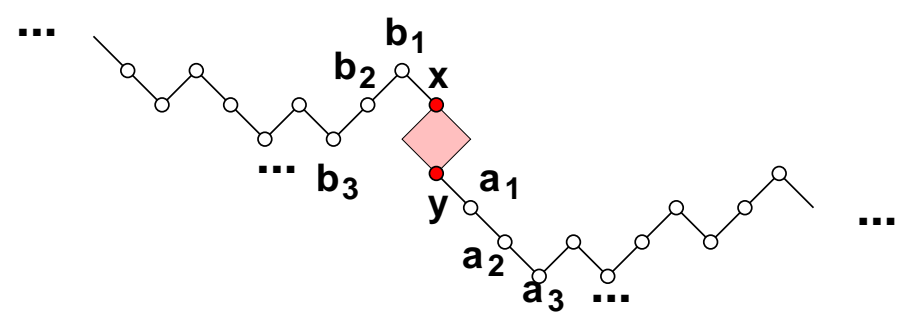

where the two uncircled vertices of the puncture bear no assignment. It is easy to show that a necessary condition for the Laurent property to still hold is that $x y=1$. Conversely, we have the following:

Theorem 6.1. The solution of the $A_{1} T$-system with a puncture, an arbitrary path of initial conditions (passing by the puncture) as above, and with $x y=1$, is a positive Laurent polynomial of the initial data.

Proof. We start by reinterpreting the fundamental relation (2.5) as a flatness condition of the form $V\left(a, b^{\prime}\right) U\left(b^{\prime}, c\right) V(b, c)^{-1} U(a, b)^{-1}=\mathbb{I}$, around each diamond where the $T$-system relation holds. We see that the matrices $V, U, V^{-1}, U^{-1}$ corresponding to the 4 possible oriented edges along the vectors $(1,-1),(1,1),(-1,1),(-1,-1)$ respectively form a flat connection on any domain made of diamonds on which the $T$-system relation holds. As a consequence, the product of corresponding matrices along the boundary of any connected domain made of diamonds where the $T$-system relation holds is the identity matrix. Due to the presence of the puncture, we may only consider domains that do not contain it. Let $(j, k)$ be a point above the initial data paths, and $j_{0}, j_{1}$ the minimum and maximum of its projection as usual. We consider the domain $D$ shaded below:

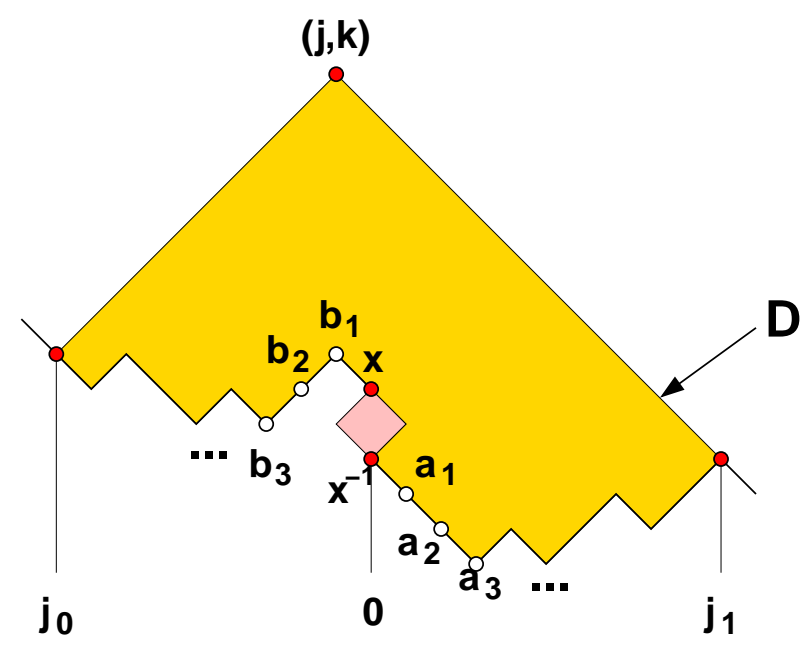

In particular, the two (topmost and bottommost) paths joining the minimum and maximum contribute the same matrix $M$, satisfying $M_{1,1} T_{j_{1}, k_{j_{1}}}=T_{j, k}$, clear from the topmost path expression of the form $U U \cdots U V V \cdots V$. The contribution from the bottommost path is the product along the initial data path (including edges of the puncture) of the 
corresponding connection matrices. These have non-negative entries that are Laurent monomials of the initial data, except for edges of the puncture namely $V(x, w) U\left(x^{-1}, w\right)^{-1}$ or $V\left(v, x^{-1}\right)^{-1} U(v, x)$. The following local situations may occur in general:
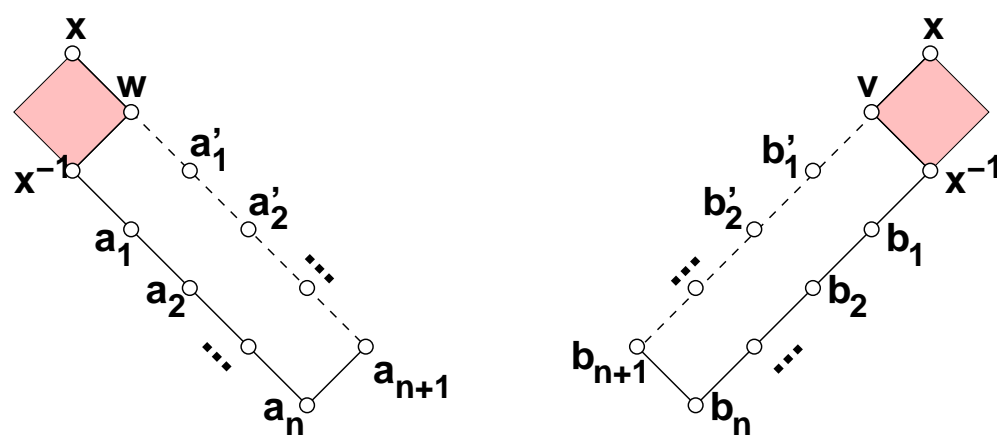

leading to matrix products along the bottommost path of the form $\cdots B_{L / R} \cdots$, where $B_{L / R}$ is the only term containing possibly negative or non-Laurent entries for the situation on the left/right, with respectively:

$$
\begin{aligned}
& B_{L}=V(x, w) U\left(x^{-1}, w\right)^{-1} V\left(x^{-1}, a_{1}\right) V\left(a_{1}, a_{2}\right) \cdots V\left(a_{n-1}, a_{n}\right) U\left(a_{n}, a_{n+1}\right) \\
& B_{R}=V\left(b_{n+1}, b_{n}\right) U\left(b_{n}, b_{n-1}\right) U\left(b_{n-1}, b_{n-2}\right) \cdots U\left(b_{1}, x^{-1}\right) V\left(x^{-1}, v\right)^{-1} U(v, x)
\end{aligned}
$$

Applying the flat connection condition to the domain made of the $n$ diamonds, we may express respectively:

$$
\begin{aligned}
& B_{L}=V(x, w) V\left(w, a_{1}^{\prime}\right) V\left(a_{1}^{\prime}, a_{2}^{\prime}\right) \cdots V\left(a_{n-1}^{\prime}, a_{n+1}\right) \\
& B_{R}=U\left(b_{n+1}, b_{n-1}^{\prime}\right) U\left(b_{n-1}^{\prime}, b_{n-2}^{\prime}\right) \cdots U\left(b_{1}^{\prime}, v\right) U(v, x)
\end{aligned}
$$

where for $a_{n}^{\prime}=a_{n+1}, a_{0}^{\prime}=w, a_{0}=x^{-1}$ and $b_{n}^{\prime}=b_{n+1}, b_{0}^{\prime}=v, b_{0}=x^{-1}$ we have the following descending recursion relations:

$$
\begin{array}{ll}
a_{m}^{\prime}=\frac{a_{m+1}^{\prime} a_{m}+1}{a_{m+1}} & (m=n-1, n-2, \ldots, 1,0) \\
b_{m}^{\prime}=\frac{b_{m+1}^{\prime} b_{m}+1}{b_{m+1}} & (m=n-1, n-2, \ldots, 1,0)
\end{array}
$$

It is straightforward to see that the $a_{m}^{\prime}, b_{m}^{\prime} m=1,2, \ldots, n-1$, are all positive Laurent polynomials of respectively $\left(x^{-1}, a_{1}, a_{2}, \ldots, a_{n+1}\right)$ and $\left(x^{-1}, b_{1}, b_{2}, \ldots, b_{n+1}\right)$. Moreover possible denominators involving $v$ or $w$ (which are not initial data) are suppressed in the products (6.1) and (6.2). As a consequence both products (6.1) and (6.2) have entries that are non-negative Laurent polynomials of the initial data. As the remainder of the matrix products along the bottommost path only involve matrices with non-negative Laurent monomial entries, the positivity follows.

We may consider more general cases where $S$ is made of possibly several chains of diamonds attached by their north/south vertices. We refer to such chains as "cuts". We observed that to guarantee the Laurent property, the N/S vertex assignments of diamonds 
along these chains must alternate between a value and its inverse $x, x^{-1}, x, x^{-1}, \ldots$ Let us consider a general situation as depicted below of arbitrary paths of initial data (empty circles) separated by chains as above, with initial data $x=x^{-1}=1$ (filled circles):



Then we conjecture that the solution to the $A_{1} T$-system is a positive Laurent polynomial of the initial data at the empty circles.

In the $A_{r}$ case, we expect the above to generalize analogously, namely that a general situation with pieces of stepped surfaces separated by pieces of walls of singular octahedra attached by their vertices $(i, j, k)$ with the same value of $j$, at which the assigned value of $T_{i, j, k}$ is 1 , leads to a solution that is a positive Laurent polynomial of the initial data along the stepped surfaces.

This generalizes the $\ell$-restricted situation of the present paper, in which we consider two infinite parallel walls of singular tetrahedra and a finite stepped surface in-between.

Acknowledgments. The authors acknowledge support by the CNRS PICS program. PDF received partial support from the ANR Grant GranMa. RK is supported by NSF grant DMS-1100929. PDF would like to thank the Mathematical Science Research Institute in Berkeley, CA and the organizers of the semester "Random Spatial Processes" for hospitality while this work was completed.

\section{References}

[1] I. Assem, C. Reutenauer and D. Smith, Friezes, Adv. Math. 225 (2010), 3134-3165. arXiv:0906.2026[math.RA]

[2] V. Bazhanov and N. Reshetikhin, Restricted solid-on-solid models connected with simply-laced algebras and conformal field theory. J. Phys. A: Math. Gen. 23 (1990) 1477-1492. 
[3] H.S.M. Coxeter, Frieze Patterns, Triangulated Polygons and Dichromatic Symmetry, in The Lighter Side of Mathematics, R.K. Guy and E. Woodrow (eds.), John Wiley \& Sons, NY, (1961) pp 15-27.

[4] P. Di Francesco, The solution of the $A_{r}$ T-system for arbitrary boundary, Elec. Jour. of Comb. Vol. 17(1) (2010) R89. arXiv: 1002.4427[math.CO]

[5] P. Di Francesco and R. Kedem, Q-systems as cluster algebras II, Lett. Math. Phys. 89 No 3 (2009) 183-216. arXiv:0803.0362[math.RT]

[6] P. Di Francesco and R. Kedem, Q-systems, heaps, paths and cluster positivity, Comm. Math. Phys. 293 No. 3 (2009) 727-802, doi:10.1007/s00220-009-0947-5. arXiv: 0811.3027[math.CO]

[7] P. Di Francesco and R. Kedem, Positivity of the T-system cluster algebra, Elec. Jour. of Comb. Vol. 16(1) (2009) R140, Oberwolfach preprint OWP 2009-21, arXiv:0908.3122[math.CO]

[8] N. Elkies, G. Kuperberg, M. Larsen and J. Propp, Alternating-Sign Matrices and Domino Tilings (Parts I and II) Jour. of Alg. Comb. Vol 1, No 2 (1992), 111-132 and Vol 1, No 3 (1992), 219-234.

[9] S. Fomin and A. Zelevinsky Cluster Algebras I. J. Amer. Math. Soc. 15 (2002), no. 2, 497-529. arXiv:math/0104151[math.RT]

[10] M. Glick, The pentagram map and Y-patterns, Adv. in Math. 227 (2011) 1019-1045. arXiv: 1005.0598[math.CO]

[11] M. Gekhtman, M. Shapiro, S. Tabachnikov, A. Vainshtein, Higher pentagram maps, weighted directed networks, and cluster dynamics, preprint arXiv: $1110.0472[$ math.QA]

[12] A. Henriques, A periodicity theorem for the octahedron recurrence, Jour. of Alg. Comb. Vol. 26 Issue 1 (2007),1-26. arXiv:math/0604289[math.CO]

[13] R. Inoue, O. Iyama, A. Kuniba, T. Nakanishi, J. Suzuki, Periodicities of T-systems and Y-systems, Nagoya Math. J. 197 (2010), 59-174, arXiv:0812.0667[math.QA]

[14] R. Inoue, O. Iyama, B. Keller, A. Kuniba, T. Nakanishi, Periodicities of $T$ and $Y$ systems, dilogarithm identities, and cluster algebras I: Type $B_{r}$, and II: Types $C_{r}, F_{4}$, and $G_{2}$, arXiv: 1001.1880[math.QA] and arXiv:1001.1881[math.QA].

[15] B. Keller, Cluster algebras, quiver representations and triangulated categories, in Triangulated categories, T. Holm, P. Jorgensen, and R. Rouquier, eds., London Mathematical Society, Lecture Note Series vol. 375, Cambridge University Press, 2010, pp. 76-160; and The periodicity conjecture for pairs of Dynkin diagrams. arXiv: 1001 .1531[math.RT]

[16] A. Knutson, T. Tao, and C. Woodward, A positive proof of the LittlewoodRichardson rule using the octahedron recurrence, Electr. J. Combin. 11 (2004) RP 61. arXiv:math/0306274[math.CO] 
[17] I. Krichever, O.Lipan, P.Wiegmann, and A. Zabrodin, Quantum Integrable Systems and Elliptic Solutions of Classical Discrete Nonlinear Equations, Comm. Math. Phys. 188 (1997) 267-304. arXiv:hep-th/9604080

[18] A. Kuniba, A. Nakanishi and J. Suzuki, Functional relations in solvable lattice models. I. Functional relations and representation theory. International J. Modern Phys. A 9 no. 30, pp 5215-5266 (1994). arXiv:hep-th/9310060

[19] B. Lindström, On the vector representations of induced matroids, Bull. London Math. Soc. 5 (1973) 85-90.

[20] I. M. Gessel and X. Viennot, Binomial determinants, paths and hook formulae, Adv. Math. 58 (1985) 300-321.

[21] H. Nakajima, t-analogs of q-characters of Kirillov-Reshetikhin modules of quantum affine algebras, Represent. Theory 7 (2003), 259-274 (electronic).

[22] D. Robbins and H. Rumsey, Determinants and Alternating Sign Matrices, Advances in Math. 62 (1986) 169-184.

[23] D. Speyer, Perfect matchings and the octahedron recurrence, J. Algebraic Comb. 25 No 3 (2007) 309-348. arXiv:math/0402452[math.CO]

[24] A. Szenes, Periodicity of Y-systems and flat connections, Lett. Math. Phys. 89 (2009), no. 3, 217-230. arXiv:math/0606177[math.RT]

[25] A.Yu. Volkov, On the periodicity conjecture for Y-systems, Comm. Math. Phys. 276 (2007), 509-517. arXiv:hep-th/0606094

[26] Al. B. Zamolodchikov, On the thermodynamic Bethe Ansatz equations for reflectionless ADE scattering theories, Phys. Lett. B253 (1991) 391-394. 